Supporting information for "Synthesis, Characterization, and Structure of Cyclopenta[c]thiophenes and Their Manganese Complexes" by Chad A. Snyder, John P. Selegue, ${ }^{*}$ Nathan C. Tice, Chad E. Wallace, Mark T. Blankenbuehler, Sean Parkin, Keith D. E. Allen, and Ryan T. Beck

\begin{tabular}{l|l|l} 
& Table of Contents & Page \\
\hline 1 & $\begin{array}{l}\text { General procedures } \\
2\end{array}$ & $\begin{array}{l}\text { Syntheses and spectroscopic data for } \mathbf{1 d}-\mathbf{h}, \mathbf{2 c}, \mathbf{f}, \mathbf{g}, \mathbf{3 a}, \mathbf{c}-\mathbf{e}, \mathbf{h}, \mathbf{4 a}, \\
\mathbf{b}, \mathbf{d}-\mathbf{h} \text { and } \mathbf{5 a}-\mathbf{h}\end{array}$ \\
3 & $\begin{array}{l}\text { Tables of crystallographic details, atomic coordinates and } \\
\text { displacement parameters, bond distances }\end{array}$ & 24
\end{tabular}

General procedures. Reactions were carried out by using standard organic synthetic techniques under air unless otherwise noted. $\mathrm{CDCl}_{3}, \mathrm{C}_{6} \mathrm{D}_{6}$ and DMSO- $\mathrm{d}_{6}$ (Cambridge Isotopes) were used without further purification. 1,2-Diacylcyclopentadienes 1a-c, ${ }^{1-5}$ 1,3-dimethylcyclopenta[c]thiophene $(2 \mathbf{a})^{4,6},\left[\mathrm{TI}\left\{1,2-\mathrm{C}_{5} \mathrm{H}_{3}(\mathrm{COPh})_{2}\right\}\right](\mathbf{4 c})^{4,5}$ and $\left[\mathrm{MnBr}(\mathrm{CO})_{5}\right]^{7}$ were prepared according to the cited methods. Thallium(I) ethoxide, $\mathrm{P}_{4} \mathrm{~S}_{10}$, n-butyllithium, 5-methyl-2-thiophenecarboxylic acid (Aldrich), carbon disulfide (EMD), acetyl chloride (EM Science), p-nitrobenzoyl chloride, p-toluoyl chloride, anisic acid, benzo[b]thiophene-2-carboxylic acid and dicyclopentadiene (Acros), [ $\left.\mathrm{Mn}_{2}(\mathrm{CO})_{10}\right]$ (Strem) were obtained commercially and used without further purification. Lawesson's reagent was purchased (Acros) or prepared by using Lawesson's procedure. ${ }^{8} \mathrm{Me}_{3} \mathrm{SnCl}$ (Acros) was sublimed prior to use. THF was dried over sodium benzophenone ketyl. 1,3-Cyclopentadiene was freshly prepared by dropping dicyclopentadiene into a silicone oil bath at ca. $200{ }^{\circ} \mathrm{C}$ and collecting the distillate.

${ }^{1} \mathrm{H}$ and ${ }^{13} \mathrm{C}\left\{{ }^{1} \mathrm{H}\right\}$ NMR spectra were recorded on a Varian Gemini-200 spectrometer at ca. $22{ }^{\circ} \mathrm{C}$ and were referenced to residual solvent peaks. Infrared 
spectra were recorded on an ATI-Mattson Galaxy ${ }^{\mathrm{TM}}$ Series 5000 FTIR spectrometer. UV-visible spectra were recorded on a Shimadzu UV-2501PC. Electron ionization (EI) mass spectra were recorded at $70 \mathrm{eV}$ on a Thermo Finnigan PolarisQ (quadrupole ion trap) at the University of Kentucky Mass Spectrometry Facility. Samples were introduced via a heated direct insertion probe. Melting points were taken on a standard Thermos-Hoover apparatus. X-ray diffraction data were collected at $173 \mathrm{~K}$ on a Nonius KappaCCD diffractometer. Elemental analyses were performed at the University of Illinois Microanalysis Laboratory or the University of Kentucky.

2. Syntheses and spectroscopic data for $1 d-h, 2 c, f, g, 3 a, c-e, h, 4 a-h$ and $5 a-h$

Synthesis of anisoyl chloride. The following is a slightly modified procedure of Petasis and Teets. ${ }^{9}$ To a $25-\mathrm{mL}$ round-bottom flask, anisic acid $(2.00 \mathrm{~g}, 13.2 \mathrm{mmol})$ was added to a stirred solution of $\mathrm{SOCl}_{2}(7.86 \mathrm{~g}, 4.81 \mathrm{~mL}, 66 \mathrm{mmol})$ in $8 \mathrm{~mL}$ of toluene. The solution was allowed to reflux for 4 hours. The volatiles were removed in vacuo to give anisoyl chloride $(2.06 \mathrm{~g}, 92.1 \%)$ as an amber oil. ${ }^{1} \mathrm{H} \mathrm{NMR}\left(200 \mathrm{MHz}, \mathrm{CDCl}_{3}, \mathbf{p p m}\right)$ $\delta 3.89(\mathrm{~s}, 3 \mathrm{H}, \mathrm{OMe}), 6.95\left(\mathrm{dt}, 2 \mathrm{H},{ }^{1} \mathrm{~J}=9.2 \mathrm{~Hz},{ }^{2} \mathrm{~J}=2.4 \mathrm{~Hz}, \mathrm{Ar}\right), 8.06\left(\mathrm{dt}, 2 \mathrm{H},{ }^{1} \mathrm{~J}=9.2 \mathrm{~Hz}\right.$, $\left.{ }^{2} \mathrm{~J}=2.4 \mathrm{~Hz}, \mathrm{Ar}\right) . \mathrm{GC} / \mathrm{MS}: \mathrm{m} / \mathrm{z} 170\left(\mathrm{M}^{+}\right), 135\left(\mathrm{M}^{+}-\mathrm{Cl}\right)$.

Synthesis of 5-methyl-2-thiophenecarbonyl chloride. To a solution of 5methyl-2-thiophenecarboxylic acid $(4.00 \mathrm{~g}, 28.1 \mathrm{mmol})$ in refluxing toluene $(16 \mathrm{~mL})$ was added $\mathrm{SOCl}_{2}(16.8 \mathrm{~g}, 10.3 \mathrm{~mL}, 141 \mathrm{mmol})$. The solution was stirred under nitrogen for 4-5 hours. The reaction mixture was cooled to room temperature and excess $\mathrm{SOCl}_{2}$ and toluene were removed in vacuo to give 5-methyl-2-thiophenecarbonyl chloride (4.05 g, $25.3 \mathrm{mmol}, 89.9 \%) .{ }^{1} \mathrm{H}$ NMR (200 MHz, $\left.\mathrm{CDCl}_{3}, \mathrm{ppm}\right) \delta 2.48(\mathrm{~s}, 3 \mathrm{H}, \mathrm{Me}), 6.79\left(\mathrm{~d},{ }^{3} \mathrm{JHH}_{\mathrm{HH}}\right.$ 
$=4 \mathrm{~Hz}, 1 \mathrm{H}, \operatorname{ArH}), 7.71\left(\mathrm{~d},{ }^{3} J_{\mathrm{HH}}=4 \mathrm{~Hz}, 1 \mathrm{H}, \operatorname{ArH}\right) .{ }^{1} \mathrm{H}$ NMR $\left(200 \mathrm{MHz}, \mathrm{C}_{6} \mathrm{D}_{6}, \mathbf{p p m}\right) \delta 1.72$ (s, 3H, Me), $6.06\left(\mathrm{~d},{ }^{3} \mathrm{~J}_{\mathrm{HH}}=4 \mathrm{~Hz}, 1 \mathrm{H}, \operatorname{ArH}\right), 7.38\left(\mathrm{~d},{ }^{3} \mathrm{~J}_{\mathrm{HH}}=4 \mathrm{~Hz}, 1 \mathrm{H}, \operatorname{ArH}\right) .{ }^{1} \mathrm{H}$ NMR (200 $\left.\mathrm{MHz}, \mathrm{C}_{6} \mathrm{D}_{6}, \mathbf{p p m}\right) \delta 1.87(\mathrm{~s}, 3 \mathrm{H}, \mathrm{Me}), 6.20\left(\mathrm{~d},{ }^{3} \mathrm{~J}_{\mathrm{HH}}=4 \mathrm{~Hz}, 1 \mathrm{H}, \operatorname{ArH}\right), 7.47\left(\mathrm{~d},{ }^{3} \mathrm{~J}_{\mathrm{HH}}=4\right.$ $\mathrm{Hz}, 1 \mathrm{H}, \mathrm{ArH}) .{ }^{13} \mathrm{C}\left\{{ }^{1} \mathrm{H}\right\}$ NMR (50 MHz, $\left.\mathrm{CDCl}_{3}, \mathbf{p p m}\right) \delta 16.2(\mathrm{Me}), 127.5(\mathrm{ArH}), 134.2$ (ArMe), $138.8(\operatorname{ArH}), 154.4(\mathrm{ArCOCl}), 159.1(\mathrm{COCl}) .{ }^{13} \mathrm{C}\left\{{ }^{1} \mathrm{H}\right\}$ NMR (50 MHz, $\left.\mathbf{C}_{6} \mathbf{D}_{6}, \mathbf{p p m}\right)$

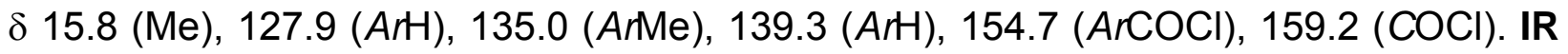
(thin film, $\left.\mathbf{c m}^{-1}\right) 1740.5(\mathrm{CO}), 2924.5\left(\mathrm{CH}_{3}\right), 3100.0(\mathrm{ArH})$. IR (CCl, $\left.\mathbf{c m}^{-1}\right) 1750(\mathrm{CO})$. MS: $\mathrm{m} / \mathrm{z} 160\left(\mathrm{M}^{+}\right), 125\left(\mathrm{M}^{+}-\mathrm{Cl}\right), 97\left(\mathrm{M}^{+}-\mathrm{COCl}\right)$.

Synthesis of benzo[b]thiophene-2-carbonyl chloride. The following is a slightly modified procedure of Petasis and Teets. ${ }^{9}$ In a $50-\mathrm{mL}$ Schlenk flask, benzo[b]thiophene-2-carboxylic acid $(2.00 \mathrm{~g}, 11.2 \mathrm{mmol})$ was added to a stirred solution of $\mathrm{SOCl}_{2}(7.86 \mathrm{~g}, 4.81 \mathrm{~mL}, 66 \mathrm{mmol})$ in $10 \mathrm{~mL}$ of toluene. The solution was allowed to reflux for 3.5 hours. The volatiles were removed in vacuo to give benzo[b]thiophene-2carbonyl chloride $(1.85 \mathrm{~g}, 82.1 \%)$ as a white solid. ${ }^{1} \mathrm{H}$ NMR $\left(200 \mathrm{MHz}, \mathrm{CDCl}_{3}, \mathrm{ppm}\right)$ $\delta$ 7.44-7.57 (m, 2H, Ar), 7.84-7.96 (m, 2H, Ar), 8.27 (s, 1H, Tp). MS(EI) m/z $196(\mathrm{M}+)$.

Synthesis of $1,2-\mathrm{C}_{5} \mathrm{H}_{3}(\mathrm{COH}-4-\mathrm{Tol})(\mathrm{CO}-4-\mathrm{Tol})$ (1d). To a $250-\mathrm{mL}$ three-necked round-bottom flask, cyclopentadiene $(5.00 \mathrm{~g}, 6.25 \mathrm{~mL}, 76.9 \mathrm{mmol})$ was slowly added to a stirred solution of n-butyllithium $(33.0 \mathrm{~mL}, 2.50 \mathrm{M}$ in hexanes, $82.5 \mathrm{mmol})$ in $75 \mathrm{~mL}$ of ethyl ether. A white precipitate of cyclopentadienyllithium immediately formed. The white suspension was stirred at $0{ }^{\circ} \mathrm{C}$ for 15 minutes. 4 -Toluoyl chloride $(7.91 \mathrm{~g}, 6.82 \mathrm{~mL}, 51.2$ mmol) was added dropwise and a bright yellow color formed immediately. The yellow suspension was allowed to warm to room temperature and stir for 2 hours. The reaction mixture was hydrolyzed with $3 \%$ acetic acid $(50 \mathrm{~mL})$. The organic layer was collected 
and the aqueous layer was exacted with ethyl ether $(3 \times 50 \mathrm{~mL})$. The combined ethyl ether extracts were combined and dried over $\mathrm{MgSO}_{4}$. The volatiles were removed in vacuo to give an orange semi-solid. Trituration with cold methanol and filtration yielded 1d (4.04 g, 52.3\%) as a yellow-orange solid. Mp: $141-143{ }^{\circ} \mathrm{C} .{ }^{1} \mathrm{H}$ NMR (200 MHz, $\left.\mathrm{CDCl}_{3}, \mathrm{ppm}\right) \delta 2.44(\mathrm{~s}, 6 \mathrm{H}, \mathrm{Me}), 6.46(\mathrm{t}, 1 \mathrm{H}, \mathrm{J}=4, \mathrm{CHCHCH}), 7.25(\mathrm{~d}, 2 \mathrm{H}, \mathrm{J}=4$, $\mathrm{CHCHCH}), 7.28\left(\mathrm{dt}, 4 \mathrm{H},{ }^{1} \mathrm{~J}=4.4,{ }^{2} \mathrm{~J}=2.2, \mathrm{Ar}\right), 7.70\left(\mathrm{dt}, 4 \mathrm{H},{ }^{1} \mathrm{~J}=4.4,{ }^{2} \mathrm{~J}=2.2, \mathrm{Ar}\right) 18.55$ (s, $1 \mathrm{H}, \mathrm{OH}) .{ }^{13} \mathrm{C}\left\{{ }^{1} \mathrm{H}\right\}$ NMR (50 MHz, $\left.\mathrm{CDCl}_{3}, \mathrm{ppm}\right) \delta 21.9(\mathrm{Me}), 122.7(\mathrm{CHCHCH}), 124.5$ (CHCHCH), 129.0 (Ar), 129.2 (CC), 130.1, 141.2, 142.1 (Ar), 185.5 (p-TolCO). IR (KBr, cm$\left.{ }^{-1}\right) 2919$ (Me), 1607 (CO). GC/MS: m/z $302\left(M^{+}\right)$. HRMS: $\left(M^{+}\right)$calcd 302.1307, obsd 302.1306. Anal. Calcd for $\mathrm{C}_{21} \mathrm{H}_{18} \mathrm{O}_{2}$ : C,83.42; $\mathrm{H}, 6.00$. Found: $\mathrm{C}, 83.40 ; \mathrm{H}, 5.91$.

Synthesis of $1,2-\mathrm{C}_{5} \mathrm{H}_{3}\left(\mathrm{COH}-4-\mathrm{MeOC}_{6} \mathrm{H}_{4}\right)\left(\mathrm{CO}-4-\mathrm{MeOC}_{6} \mathrm{H}_{4}\right)$ (1e). To a $250-\mathrm{mL}$ Schlenk flask at $0{ }^{\circ} \mathrm{C}$, cyclopentadiene $(5.04 \mathrm{~g}, 6.28 \mathrm{~mL}, 77.5 \mathrm{mmol})$ was added dropwise to a stirred solution of n-butyllithium (33 mL of $2.5 \mathrm{M}, 82.5 \mathrm{mmol}$ ) in $75 \mathrm{~mL}$ of ethyl ether. A white precipitate of cyclopentadienyllithium immediately formed. The white suspension was stirred at $0{ }^{\circ} \mathrm{C}$ for 15 minutes. To the reaction mixture, anisoyl chloride (6.98 $\mathrm{g}, 51.67 \mathrm{mmol})$ in $20 \mathrm{~mL}$ of ethyl ether was added dropwise. A bright yellow color formed immediately. The mixture was allowed to warm to room temperature and stir for 2 hours. The reaction mixture was hydrolyzed with $3 \%$ acetic acid $(50 \mathrm{~mL})$. The organic layer was collected and the aqueous layer was exacted with ethyl ether $(3 \times 50 \mathrm{~mL})$. The combined ethyl ether extracts were combined and dried over $\mathrm{MgSO}_{4}$. The volatiles were removed in vacuo to give a yellow-brown semi-solid. Trituration with cold methanol gave $1 \mathrm{e}(4.23 \mathrm{~g} 49.0 \%)$ as a dark yellow solid. Mp: $151-153{ }^{\circ} \mathrm{C} .{ }^{1} \mathrm{H}$ NMR (200 MHz, $\left.\mathrm{CDCl}_{3}, \mathrm{ppm}\right) \delta 3.20(\mathrm{~s}, 6 \mathrm{H}, \mathrm{MeO}), 6.49(\mathrm{t}, 1 \mathrm{H}, \mathrm{J}=4 \mathrm{~Hz}, \mathrm{CHCHCH}), 6.66(\mathrm{~d}, 4 \mathrm{H}, \mathrm{J}=9.2$ 
$\mathrm{Hz}, \mathrm{Ar}), 7.32(\mathrm{~d}, 2 \mathrm{H}, \mathrm{J}=4 \mathrm{~Hz}, \mathrm{CHCHCH}), 7.78(\mathrm{~d}, 4 \mathrm{H}, \mathrm{J}=9.2 \mathrm{~Hz}, \mathrm{Ar}), 19.45(\mathrm{~s}, 1 \mathrm{H}$, $\mathrm{OH}) .{ }^{13} \mathrm{C}\left\{{ }^{1} \mathrm{H}\right\}$ NMR (50 MHz, $\left.\mathrm{CDCl}_{3}, \mathbf{p p m}\right) \delta 55.7(\mathrm{MeO}), 113.8(\mathrm{CHCHCH}), 122.3$ $(\mathrm{CHCHCH}), 124.3(\mathrm{CC}), 130.4,132.3,140.5,162.7(\mathrm{Ar}), 184.8\left(\mathrm{COp}-\mathrm{MeOC}_{6} \mathrm{H}_{4}\right) . \mathbf{I R}$ (KBr, cm $\left.{ }^{-1}\right) 3437$ (vb, OH), 1603 (CO), 1255 (MeO). HRMS: (M+) calcd 334.1205, obsd 334.1194. Anal. Calcd for $\mathrm{C}_{21} \mathrm{H}_{18} \mathrm{O}_{4}$ : C, 75.43; $\mathrm{H}, 5.43$. Found: $\mathrm{C}, 75.16 ; \mathrm{H}, 5.63$.

Synthesis of 1,2- $\mathrm{C}_{5} \mathrm{H}_{3}(\mathrm{CTpOH})(\mathrm{COTp})$ (1f). Cyclopentadiene $(5.04 \mathrm{~g}, 6.28 \mathrm{~mL}$, $77.5 \mathrm{mmol}$ ) was added dropwise to a cooled solution $\left(0^{\circ} \mathrm{C}\right)$ of $\mathrm{n}$-butyllithium $(30.8 \mathrm{~mL}$ of $2.6 \mathrm{M}, 80 \mathrm{mmol})$ in ethyl ether $(150 \mathrm{~mL})$. A white precipitate of cyclopentadienyllithium was formed. The suspension was stirred for 15 minutes and 2-thiophenecarbonyl chloride $(7.34 \mathrm{~g}, 5.35 \mathrm{~mL}, 50 \mathrm{mmol})$ was added dropwise. A bright yellow color formed immediately. Stirring was continued for 45 minutes at room temperature. The reaction mixture was hydrolyzed with dilute $(3 \%)$ acetic acid $(70 \mathrm{~mL})$. The ether layer was separated, and the aqueous layer was extracted twice more with ethyl ether $(2 \times 250$ $\mathrm{mL}$ ). The combined ether extracts were dried over $\mathrm{MgSO}_{4}$. The solvent was removed under reduced pressure leaving a brick-red solid. Recrystallization from methanol yielded $6.09 \mathrm{~g}, 21.4 \mathrm{mmol}$ of orange solid (43\%). Mp: $102-103^{\circ} \mathrm{C} .{ }^{1} \mathrm{H}$ NMR (200 MHz, $\left.\mathrm{CDCl}_{3}, \mathrm{ppm}\right) \delta 6.54(\mathrm{t}, 1 \mathrm{H}, \mathrm{J}=4.0 \mathrm{~Hz}, \mathrm{CHCHCH}), 7.18(\mathrm{dd}, 2 \mathrm{H}, \mathrm{J}=4.9,4.0 \mathrm{~Hz}, \mathrm{Tp})$, $7.66(\mathrm{~d}, 2 \mathrm{H}, \mathrm{J}=4.0, \mathrm{CHCHCH}$, overlapping $\mathrm{d}, 2 \mathrm{H}, \mathrm{J}=1.1,4.9 \mathrm{~Hz}, \mathrm{Tp}), 7.82(\mathrm{dd}, 2 \mathrm{H}, \mathrm{J}=$ 4.0, $1.1 \mathrm{~Hz}, \mathrm{Tp}), 18.27(\mathrm{~s}, 1 \mathrm{H}, \mathrm{OH}) .{ }^{13} \mathrm{C}\left\{{ }^{1} \mathrm{H}\right\} \mathrm{NMR}\left(100 \mathrm{MHz}, \mathrm{CDCl}_{3}, \mathbf{p p m}\right) \delta 176(\mathrm{CO})$, 140.6 (Tp CS), 139.2 (Tp CH), 133.8 (Tp CH), 132.5 (Tp CH), $127.8(\mathrm{CHCHCH}), 123.9$ (CC), $123.1(\mathrm{CHCHCH})$. IR (KBr, cm-1) $3169(\mathrm{C}-\mathrm{H}), 3120(\mathrm{C}-\mathrm{H})$, 1397. MS (EI): calcd 286.01 $\left(\mathrm{M}^{+}\right)$, obsd 286.0. Anal. Calcd for $\mathrm{C}_{15} \mathrm{H}_{10} \mathrm{O}_{2} \mathrm{~S}_{2}$ : $\mathrm{C}, 62.91 ; \mathrm{H}, 3.52$. Found: $\mathrm{C}$, 63.45; H, 3.44. 
Synthesis of $1,2-\mathrm{C}_{5} \mathrm{H}_{3}\left(\mathrm{CO}-2-\mathrm{C}_{4} \mathrm{H}_{2} \mathrm{~S}-5-\mathrm{Me}\right)\left(\mathrm{COH}-2-\mathrm{C}_{4} \mathrm{H}_{2} \mathrm{~S}-5-\mathrm{Me}\right)(\mathrm{g})$. To a $250-$ $\mathrm{mL}$ Schlenk flask at $0{ }^{\circ} \mathrm{C}$, cyclopentadiene $(2.59 \mathrm{~g}, 3.23 \mathrm{~mL}, 39.2 \mathrm{mmol})$ was added dropwise to a cooled solution $\left(0^{\circ} \mathrm{C}\right)$ of n-butyllithium $(16.2 \mathrm{~mL}$ of $2.50 \mathrm{M}, 23.4 \mathrm{~g}, 40.5$ $\mathrm{mmol})$ in ethyl ether $(120 \mathrm{~mL})$. A white precipitate of cyclopentadienyllithium was formed immediately. The suspension was stirred for 15 minutes and 5-methyl-2thiophenecarbonyl chloride $(4.05 \mathrm{~g}, 3.15 \mathrm{~mL}, 28.1 \mathrm{mmol})$ was added dropwise. A bright yellow color formed immediately. The solution was stirred overnight at room temperature. The reaction mixture was hydrolyzed with dilute $(3 \%)$ acetic acid $(80 \mathrm{~mL})$. The organic layer was separated, and the aqueous layer was extracted twice more with ethyl ether $(2 \times 40 \mathrm{~mL})$. The combined ethyl ether extracts were dried $\left(\mathrm{MgSO}_{4}\right)$ and removed under reduced pressure to leave a red semi-solid. The solid was triturated with an acetone/methanol mixture $(50 / 50)(5 \times 10 \mathrm{~mL})$ to leave $1 \mathrm{~g}(2.08 \mathrm{~g}, 6.62 \mathrm{mmol}$, $51.3 \%)$ as a bright red solid. Mp: $120-122{ }^{\circ} \mathrm{C} .{ }^{1} \mathrm{H}$ NMR $\left(200 \mathrm{MHz}, \mathrm{CDCl}_{3}, \mathrm{ppm}\right) \delta 2.57$ (s, 6H, 2Me), $6.50\left(\mathrm{t},{ }^{3} \mathrm{~J}_{\mathrm{HH}}=4.03 \mathrm{~Hz}, 1 \mathrm{H}, \mathrm{CHCHCH}\right), 6.84\left(\mathrm{~d},{ }^{3} \mathrm{~J}_{\mathrm{HH}}=3.66 \mathrm{~Hz}, 2 \mathrm{H}, 2 \mathrm{ArH}\right)$, $7.61\left(\mathrm{~d},{ }^{3} \mathrm{~J}_{\mathrm{HH}}=4.03 \mathrm{~Hz}, 2 \mathrm{H}, \mathrm{CHCHCH}\right), 7.66\left(\mathrm{~d}, 2 \mathrm{H},{ }^{3} \mathrm{~J}_{\mathrm{HH}}=3.66 \mathrm{~Hz}, 2 \mathrm{ArH}\right), 18.3(\mathrm{~s}, 1 \mathrm{H}$, $\mathrm{OH}) .{ }^{1} \mathrm{H}$ NMR (200 MHz, DMSO, ppm) $\delta 2.57,(\mathrm{~s}, 6 \mathrm{H}, 2 \mathrm{Me}), 6.57\left(\mathrm{t},{ }^{3} \mathrm{~J}_{\mathrm{HH}}=4.0 \mathrm{~Hz}, 1 \mathrm{H}\right.$, $\mathrm{CHCHCH}), 7.04\left(\mathrm{~d},{ }^{3} \mathrm{~J}_{\mathrm{HH}}=3.6 \mathrm{~Hz}, 2 \mathrm{H}, 2 \mathrm{ArH}\right), 7.67\left(\mathrm{~d},{ }^{3} \mathrm{~J}_{\mathrm{HH}}=4.03 \mathrm{~Hz}, 2 \mathrm{H}, \mathrm{CHCHCH}\right)$, $7.82\left(\mathrm{~d}, 2 \mathrm{H},{ }^{3} \mathrm{~J}_{\mathrm{HH}}=3.6 \mathrm{~Hz}, 2 \mathrm{ArH}\right), 18.30(\mathrm{~s}, 1 \mathrm{H}, \mathrm{OH}) .{ }^{13} \mathrm{C}\left\{{ }^{1} \mathrm{H}\right\}$ NMR $\left(50 \mathrm{MHz}, \mathrm{CDCl}_{3}\right.$, ppm) $\delta 15.8(\mathrm{Me}), 122.5(\mathrm{CHCHCH}), 123.6(\mathrm{CHCCO}), 126.5(\mathrm{ArH}), 134.6(\mathrm{CHCHCH})$, 138.0 (ArH), 138.3, 148.5 (Ar), 176.2 (CO). $\left.{ }^{13} \mathrm{C}^{1}{ }^{1} \mathrm{H}\right\}$ NMR (50 MHz, DMSO, ppm) $\delta$ $15.4(\mathrm{Me}), 122.7(\mathrm{CHCHCH}), 123.5(\mathrm{CHCCO}), 127.7(\mathrm{ArH}), 138.5(\mathrm{CHCHCH}), 135.6$ (ArH), 137.0, 149.3 (Ar), 175.1 (CO). IR (KBr, cm ${ }^{-1}$ ) $1531.7(\mathrm{CO}), 2914.4(\mathrm{Me}), 3068.0$ 3088.5 (ArH). HRMS: $\left(\mathrm{M}^{+}\right)$calcd 314.0435, obsd 314.0433. GC/MS: m/z $314\left(\mathrm{M}^{+}\right), 216$ 
$\left(\mathrm{M}^{+}-\mathrm{C}_{4} \mathrm{H}_{2} \mathrm{SMe}\right), 187\left(\mathrm{M}^{+}-\mathrm{COC}_{4} \mathrm{H}_{2} \mathrm{SMe}\right), 119\left(\mathrm{M}^{+}-2 \mathrm{C}_{4} \mathrm{H}_{2} \mathrm{SMe}\right), 63\left(\mathrm{M}^{+}-2 \mathrm{COC}_{4} \mathrm{H}_{2} \mathrm{SMe}\right)$.

Anal. Calc for $\mathrm{C}_{17} \mathrm{H}_{14} \mathrm{O}_{2} \mathrm{~S}_{2}$ : C, 64.97; $\mathrm{H}, 4.46$. Found: $\mathrm{C}, 64.98 ; \mathrm{H}, 4.48$.

Synthesis of $1,2-\mathrm{C}_{5} \mathrm{H}_{3}\left(\mathrm{COHC}_{8} \mathrm{H}_{5} \mathrm{~S}\right)\left(\mathrm{COC}_{8} \mathrm{H}_{5} \mathrm{~S}\right)(1 \mathrm{~h})$. To a $250-\mathrm{mL}$, three-necked round-bottom flask, cyclopentadiene $(0.908 \mathrm{~g}, 1.13 \mathrm{~mL}, 14.0 \mathrm{mmol})$ was added dropwise to a stirred solution of $n$-butyllithium $(5.59 \mathrm{~mL}$ of $2.5 \mathrm{M}, 14.0 \mathrm{mmol})$ in $75 \mathrm{~mL}$ of ethyl ether. A white precipitate of cyclopentadienyllithium immediately formed. The white suspension was stirred at $0{ }^{\circ} \mathrm{C}$ for 15 minutes. To the reaction mixture, benzo[b]thiophene-2-carbonyl chloride $(1.66 \mathrm{~g}, 8.45 \mathrm{mmol})$ was added. A bright orange color formed immediately. The mixture was allowed to warm to room temperature and stir for 1 hour. The reaction mixture was hydrolyzed with $3 \%$ acetic acid $(50 \mathrm{~mL})$ and an orange precipitate formed. The precipitate was filtered and dried in vacuo to give $\mathbf{1 h}$ as a bright orange solid (1.54 g, 70.9\%). Mp: $225-226{ }^{\circ} \mathrm{C} .{ }^{1} \mathrm{H}$ NMR $\left(200 \mathrm{MHz}, \mathrm{CDCl}_{3}\right.$, ppm) $\delta 6.62(\mathrm{t}, 1 \mathrm{H}, \mathrm{J}=4.0 \mathrm{~Hz}, \mathrm{CHCHCH}), 7.42-7.48(\mathrm{~m}, 4 \mathrm{H}, \mathrm{Ar}), 7.78(\mathrm{~d}, 2 \mathrm{H}, \mathrm{J}=4.0$ $\mathrm{Hz}, \mathrm{CHCHCH}), 7.78(\mathrm{~m}, 2 \mathrm{H}, \mathrm{Ar}), 8.10(\mathrm{~s}, 2 \mathrm{H}, \mathrm{Tp}), 18.25(\mathrm{~s}, 1 \mathrm{H}, \mathrm{OH}) .{ }^{13} \mathrm{C}\left\{{ }^{1} \mathrm{H}\right\}$ NMR (50 $\left.\mathrm{MHz}, \mathrm{CDCl}_{3}, \mathbf{p p m}\right) \delta 122.9(\mathrm{CHCHCH}), 124.2(\mathrm{CHCHCH}), 124.9(\mathrm{CC}), 125.3,125.3$, 125.7, 131.1, 139.1, 140.6, 142.4 (Ar), 192.2 (CO). IR (KBr, cm$\left.{ }^{-1}\right) 3426$ (vb, OH), 1590 (CO). HRMS: $\left(\mathrm{M}^{+}\right)$calcd 386.0435, obsd 386.0429. Anal. Calcd for $\mathrm{C}_{23} \mathrm{H}_{14} \mathrm{~S}_{2} \mathrm{O}_{2}: \mathrm{C}$, 71.48; H, 3.65. Found: C, 70.40; H, 3.34.

Synthesis of 1,3-diphenyl-4H-cyclopenta[c]thiophene (2c). To a solution of 1,2- $\mathrm{C}_{5} \mathrm{H}_{3}(\mathrm{CPhOH})(\mathrm{COPh})(\mathbf{1 c}, 0.500 \mathrm{~g}, 1.82 \mathrm{mmol})$ in dry toluene $(90 \mathrm{~mL})$ was added Lawesson's reagent $(0.996 \mathrm{~g}, 2.46 \mathrm{mmol})$. The solution was heated to reflux and stirred for 8 hours. After cooling to room temperature, the solution was reduced to an oil via rotary evaporation and further dried on the Schlenk line for 2 hours. The oil was cooled 
over liquid nitrogen then extracted using cold pentane. The organics were filtered and volatiles removed via rotary evaporation giving an oil. The crude oil was purified by column chromatography on silica (30/70 toluene/pentane), yielding $\mathbf{2 c}$ as a creamcolored solid. Rechromatographing the second fluorescent fraction, containing a mixture of $2 \mathrm{c}$ and unknown compound, on silica (96/4 hexane/ethyl ether) yielded additional $2 \mathrm{c}$ (combined yield $113 \mathrm{mg}, 22.5 \%$ ). The white solid was recrystallized from hot methanol to yield clear plate-like crystals. $\mathrm{Mp}: 80-83{ }^{\circ} \mathrm{C} .{ }^{1} \mathrm{H}$ NMR $\left(200 \mathrm{MHz}, \mathrm{CDCl}_{3}, \mathbf{p p m}\right) \delta 3.44$ $\left(\mathrm{dd},{ }^{4} \mathrm{~J}_{\mathrm{AC}}=2.2 \mathrm{~Hz}, 2 \mathrm{H}, \mathrm{CH}\right.$ ), $6.69\left(\mathrm{dt},{ }^{3} \mathrm{~J}_{\mathrm{AB}}=2.2 \mathrm{~Hz},{ }^{3} \mathrm{~J}_{\mathrm{BC}}=5.6 \mathrm{~Hz}, 1 \mathrm{H}, \mathrm{CH}_{B}=\mathrm{CH}_{\mathrm{C}}\right.$ ), 6.97 $\left(\mathrm{dt},{ }^{4} J_{\mathrm{AC}}=2.2 \mathrm{~Hz},{ }^{3} J_{\mathrm{BC}}=5.6 \mathrm{~Hz}, 1 \mathrm{H}, \mathrm{CH}_{\mathrm{B}}=\mathrm{CH}_{\mathrm{C}}\right), 7.24-7.31\left(\mathrm{~m}, 2 \mathrm{H}, H_{\text {para }}\right), 7.37-7.45$ (m, 4H, $\left.H_{\text {meta }}\right), 7.61-7.66\left(\mathrm{~m}, 4 \mathrm{H}, H_{\text {ortho }}\right) .{ }^{13} \mathrm{C}\left\{{ }^{1} \mathrm{H}\right\}$ NMR (50 MHz, $\left.\mathrm{CDCl}_{3}, \mathrm{ppm}\right) \delta 34.6$ $\left(\mathrm{CH}_{2}\right), 126.1\left(\mathrm{CH}_{2} \mathrm{CHCH}\right), 126.1,126.8,126.9,127.0,127.9,128.9,129.0,131.9,134.5$, 134.8, 143.1, $147.3(\mathrm{Ar}), 139.5\left(\mathrm{CH}_{2} \mathrm{CHCH}\right)$. IR (thin film, cm $\left.{ }^{-1}\right) 3054(\mathrm{Ar}-\mathrm{H}), 2922(\mathrm{C}-$ H). HRMS: $\left(M^{+}\right)$calcd 274.0816, obsd 274.0817. GC/MS: m/z $274\left(M^{+}\right), 197\left(M^{+}-P h\right)$, $120\left(\mathrm{M}^{+}-2 \mathrm{Ph}\right)$. Anal. Calc for $\mathrm{C}_{19} \mathrm{H}_{14} \mathrm{~S}: \mathrm{C}, 83.17 ; \mathrm{H}, 5.14$; Found: $\mathrm{C}, 82.50 ; \mathrm{H}, 5.13$. Numerous attempts to obtain a microanalysis in closer agreement with the proposed formula were unsuccessful.

Synthesis of $1,3-$ dithienyl-4H-cyclopenta[c]thiophene (2f). To a solution of 1,2- $\mathrm{C}_{5} \mathrm{H}_{3}(\mathrm{CTpOH})(\mathrm{COTp})(\mathbf{1 f}, 0.834 \mathrm{~g}, 2.92 \mathrm{mmol})$ in dry toluene $(90 \mathrm{~mL})$ was added Lawesson's reagent $(1.59 \mathrm{~g}, 3.94 \mathrm{mmol})$. The solution was heated to reflux and allowed to stir for $8 \mathrm{~h}$. After cooling to room temperature, the solution was reduced to an oil via rotary evaporation and further dried on the Schlenk line for 2 hours. The oil was cooled over liquid nitrogen then extracted using cold pentane. The organics were filtered and volatiles removed via rotary evaporation giving an oil. The crude oil was purified by 
column chromatography on silica (30/70 toluene/pentane), to yield $\mathbf{2 f}$ as a creamcolored solid. Rechromatographing the second fluorescent fraction, containing a mixture of $2 \mathbf{f}$ and unknown compound, on silica (96/4 hexane/ethyl ether) yielded $2 \mathbf{f}$ (combined yield $0.125 \mathrm{~g}, 15.0 \%$ ). The solid was recrystallized from hot methanol. Mp: $98-101^{\circ} \mathrm{C}$. ${ }^{1} \mathrm{H}$ NMR (200 MHz, CDCl 3 , ppm) $\delta 3.34\left(\mathrm{dd},{ }^{4} \mathrm{~J}_{\mathrm{AC}}=2.2 \mathrm{~Hz}, 2 \mathrm{H}, \mathrm{CH}_{2}\right), 6.67\left(\mathrm{dt},{ }^{3} \mathrm{~J}_{\mathrm{AB}}=2.2\right.$ $\left.\mathrm{Hz},{ }^{3} \mathrm{~J}_{\mathrm{BC}}=5.8 \mathrm{~Hz}, \mathrm{CH}_{\mathrm{B}}=\mathrm{CH}_{\mathrm{C}}\right), 6.93\left(\mathrm{dt},{ }^{4} \mathrm{~J}_{\mathrm{AC}}=2.2 \mathrm{~Hz},{ }^{3} \mathrm{~J}_{\mathrm{BC}}=5.8 \mathrm{~Hz}, \mathrm{CH}_{\mathrm{B}}=\mathrm{CH}_{\mathrm{C}}\right), 7.02-$ $7.23(\mathrm{~m}, 6 \mathrm{H}, \mathrm{Tp}) .{ }^{13} \mathrm{C}\left\{{ }^{1} \mathrm{H}\right\}$ NMR (50 MHz, $\left.\mathrm{CDCl} 3, \mathrm{ppm}\right) \delta 34.3\left(\mathrm{CH}_{2}\right), 127.4\left(\mathrm{CH}_{2} \mathrm{CHCH}\right)$, 120.6, 123.6, 123.8, 124.4, 124.5, 127.8, 128.0, 136.9, 142.6, 146.7 (Ar), 139.8 $\left(\mathrm{CH}_{2} \mathrm{CHCH}\right)$. IR (KBr, cm $\left.{ }^{-1}\right) 3067(\mathrm{Ar}-\mathrm{H}), 2924(\mathrm{C}-\mathrm{H})$. HRMS: $\left(\mathrm{M}^{+}\right)$calcd 285.9945, obsd 285.9951. GC/MS: m/z $286\left(M^{+}\right), 203\left(M^{+}-T p\right), 120\left(M^{+}-2 T p\right)$. Anal. Calc for $\mathrm{C}_{15} \mathrm{H}_{10} \mathrm{~S}_{3}: \mathrm{C}, 62.90 ; \mathrm{H}, 3.50$. Found: $\mathrm{C}, 63.24 ; \mathrm{H}, 3.85$.

Synthesis of 1,3-bis(5-methylthien-2-yl)-4H-cyclopenta[c]thiophene (2g). To a solution of $1,2-\mathrm{C}_{5} \mathrm{H}_{3}\left(\mathrm{CO}-2-\mathrm{C}_{4} \mathrm{H}_{2} \mathrm{~S}-5-\mathrm{Me}\right)\left(\mathrm{COH}-2-\mathrm{C}_{4} \mathrm{H}_{2} \mathrm{~S}-5-\mathrm{Me}\right)(\mathbf{1 g}, 1.00 \mathrm{~g}, 3.18 \mathrm{mmol})$ in dry toluene $(40 \mathrm{~mL})$ was added Lawesson's reagent $(1.74 \mathrm{~g}, 4.30 \mathrm{mmol})$. The solution was heated to reflux and allowed to stir for $8 \mathrm{~h}$. After cooling to room temperature, the solution was reduced to an oil via rotary evaporation and further dried on the Schlenk line for 2 hours. The oil was cooled over liquid nitrogen then extracted using cold pentane. The organics were filtered and volatiles removed via rotary evaporation giving an oil. The crude oil was purified by column chromatography on silica (30/70 toluene/pentane), to yield $\mathbf{2} \mathbf{g}$ as a cream solid. Rechromatographing the second fluorescent fraction, containing a mixture of $\mathbf{2 g}$ and unknown compound, on silica (96/4 hexane/ethyl ether) yielded $\mathbf{2 g}$ (combined yield $0.084 \mathrm{~g}, 8.40 \%$ ). Mp: $110-111^{\circ} \mathrm{C} .{ }^{1} \mathbf{H}$ NMR (200 MHz, $\left.\mathrm{CDCl}_{3}, \mathrm{ppm}\right) \delta 2.48(\mathrm{br} \mathrm{s}, 6 \mathrm{H}, 2 \mathrm{Me}), 3.29\left(\mathrm{dd},{ }^{4} \mathrm{~J}_{\mathrm{AC}}=2.2 \mathrm{~Hz}, 2 \mathrm{H}, \mathrm{CH}_{2}\right)$, 
$6.62\left(\mathrm{dt},{ }^{3} \mathrm{~J}_{\mathrm{AB}}=2.2 \mathrm{~Hz},{ }^{3} \mathrm{~J}_{\mathrm{BC}}=5.8 \mathrm{~Hz}, 1 \mathrm{H}, \mathrm{CH}_{B}=\mathrm{CH}_{\mathrm{C}}\right), 6.64-6.68(\mathrm{~m}, 2 \mathrm{H}, \mathrm{ArH}), 6.87(\mathrm{dt}$, $\left.{ }^{4} J_{A C}=2.2 \mathrm{~Hz},{ }^{3} J_{B C}=5.8 \mathrm{~Hz}, 1 \mathrm{H}, \mathrm{CH}_{\mathrm{B}}=\mathrm{CH}_{\mathrm{C}}\right), 6.90-6.93(\mathrm{~m}, 2 \mathrm{H}, \operatorname{ArH}) \cdot{ }^{13} \mathrm{C}\left\{{ }^{1} \mathrm{H}\right\} \mathrm{NMR}(50$ $\left.\mathbf{M H z}, \quad \mathbf{C D C l}_{3}, \mathbf{p p m}\right) \delta$ 15.3, $34.2(\mathrm{Me}), 29.7\left(\mathrm{CH}_{2}\right), 127.4\left(\mathrm{CH}_{2} \mathrm{CHCH}\right), 139.3$ $\left(\mathrm{CH}_{2} \mathrm{CHCH}\right), 120.4,123.3,123.5,124.8,125.8,134.7,134.8,139.0,139.2,141.7$ 145.9. IR (KBr, cm $\left.{ }^{-1}\right) 3024(\mathrm{Ar}-\mathrm{H}), 2953,2856(\mathrm{Me}), 2924(\mathrm{C}-\mathrm{H})$. HRMS: (M+) calcd 314.0258, obsd 314.0259. GC/MS: m/z $314\left(M^{+}\right), 299\left(M^{+}-M e\right), 281\left(M^{+}-H S\right), 247\left(M^{+}-\right.$ 2HS). Anal. Calc for $\mathrm{C}_{17} \mathrm{H}_{14} \mathrm{O}_{2} \mathrm{~S}_{2}$ : C, 64.97; $\mathrm{H}, 4.46$. Found: $\mathrm{C}, 64.92 ; \mathrm{H}, 4.45$.

Preparation of [TI\{1,2- $\left.\left.\mathrm{C}_{5} \mathrm{H}_{3}(\mathrm{COMe})_{2}\right\}\right]$ (4a). Thallium(I) ethoxide (692 mg, 2.78 mmol), weighed out in a tared syringe, was added to a solution of 1,2$\mathrm{C}_{5} \mathrm{H}_{3}(\mathrm{CMeOH})(\mathrm{COMe})(\mathbf{1 a})(360 \mathrm{mg}, 2.52 \mathrm{mmol}$ ) in THF (about $30 \mathrm{~mL}$ ). A bright yellow solid precipitated immediately. The solution was stirred for 2 hours. The precipitate was filtered and washed with $5 \mathrm{~mL}$ of cold ethyl ether affording $\left[\mathrm{Tl}\left\{1,2-\mathrm{C}_{5} \mathrm{H}_{3}(\mathrm{COMe})_{2}\right\}\right](4 \mathrm{a}$, $633 \mathrm{mg}, 75 \%)$ as a gray, air-stable powder. $\mathbf{M p :} \sim 160{ }^{\circ} \mathrm{C}$ dec. ${ }^{1} \mathbf{H}$ NMR (200 MHz, DMSO-d 6 , ppm) $\delta 2.27(\mathrm{~s}, 6 \mathrm{H}, \mathrm{Me}), 5.60(\mathrm{t}, 1 \mathrm{H}, \mathrm{J}=3.3 \mathrm{~Hz}, \mathrm{CHCHCH}), 6.40(\mathrm{~d}, 2 \mathrm{H}, \mathrm{J}=$ 3.3 Hz, CHCHCH). ${ }^{13} \mathrm{C}\left\{{ }^{1} \mathrm{H}\right\}$ NMR (100 MHz, DMSO-d 6, ppm) $\delta 29.1(\mathrm{Me}), 109.7$ ( $\mathrm{CHCHCH}), 121.3(\mathrm{CHCHCH}), 125.6(\mathrm{CC}), 192.6(\mathrm{CO})$. IR (KBr, cm$\left.{ }^{-1}\right) 1626(\mathrm{CO})$, 1374. MS (+El): $\mathrm{m} / \mathrm{z}$ calcd 354.1( $\left.\mathrm{M}^{+}\right)$, obsd 354.1. Anal. Calc for $\mathrm{C}_{9} \mathrm{H}_{9} \mathrm{O}_{2} \mathrm{Tl}: \mathrm{C}, 30.58$; $\mathrm{H}, 2.57$. Found $\mathrm{C}, 30.33 ; \mathrm{H}, 2.52$.

Synthesis of $\left[\mathrm{TI}\left\{1,2-\mathrm{C}_{5} \mathrm{H}_{3}\left(\mathrm{CO}^{t} \mathrm{Bu}\right)_{2}\right\}\right]$ (4b). Thallium(I) ethoxide (692 mg, 2.78 mmol) was added to a solution of 1,2- $\mathrm{C}_{5} \mathrm{H}_{3}\left(\mathrm{C}^{\mathrm{t}} \mathrm{BuOH}\right)\left(\mathrm{CO} \mathrm{Cu}^{\mathrm{B} u}\right)(\mathbf{1 b})(550 \mathrm{mg}, 2.52 \mathrm{mmol})$ in THF. A bright yellow solid precipitated immediately. The solution was stirred for 3 hours. The precipitate was filtered and washed with $5 \mathrm{~mL}$ of cold ethyl ether affording 
$\left[\mathrm{TI}\left\{1,2-\mathrm{C}_{5} \mathrm{H}_{3}\left(\mathrm{CO}^{\mathrm{B}} \mathrm{Bu}\right)_{2}\right\}\right](4 \mathrm{~b}, 940 \mathrm{mg}, 85 \%)$, which sublimes at $130^{\circ} \mathrm{C} / 0.01 \mathrm{~mm} \mathrm{Hg}$ with some decomposition. Mp: $\sim 180-190{ }^{\circ} \mathrm{C}$ dec. ${ }^{1} \mathrm{H}$ NMR $\left(200 \mathrm{MHz}, \mathrm{CDCl}_{3}, \mathbf{p p m}\right) \delta 1.14(\mathrm{~s}$, $6 \mathrm{H}, \mathrm{Me}), 5.57(\mathrm{t}, 1 \mathrm{H}, \mathrm{J}=3.3 \mathrm{~Hz}, \mathrm{CHCHCH}), 5.9(\mathrm{~d}, 2 \mathrm{H}, \mathrm{J}=3.3 \mathrm{~Hz}, \mathrm{CHCHCH}) .{ }^{13} \mathrm{C}\left\{{ }^{1} \mathrm{H}\right\}$

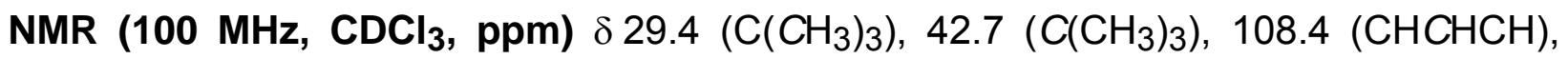
$110.3(\mathrm{CHCHCH}), 126.6(\mathrm{CC}), 206.5(\mathrm{CO}) . \mathbf{I R}\left(\mathrm{KBr}, \mathbf{c m}^{-1}\right) 2959(\mathrm{C}-\mathrm{H}), 1575(\mathrm{CO})$, 1335. MS (+EI) $\mathrm{m} / \mathrm{z}$ calcd $438.1\left(\mathrm{M}^{+}\right)$, obsd 438.1. Anal. Calc for $\mathrm{C}_{15} \mathrm{H}_{21} \mathrm{O}_{2} \mathrm{Tl}: \mathrm{C}, 41.16$; $H, 4.84 \%$. Found: C, 41.07; H, 4.78 .

Synthesis of $\left[\mathrm{TI}\left\{\mathbf{1}, \mathbf{2}-\mathrm{C}_{5} \mathrm{H}_{3}(\mathrm{CO}-4-\mathrm{Tol})_{2}\right\}\right](\mathbf{4 d})$. To a $125-\mathrm{mL}$ Schlenk flask, TIOEt (826 $\mathrm{mg}, 3.31 \mathrm{mmol})$ was added via syringe to a stirred solution of $1 \mathbf{d}(1.00 \mathrm{~g}, 3.31$ $\mathrm{mmol}$ ) in $40 \mathrm{~mL}$ of THF. A yellow precipitate immediately formed. The suspension was allowed to stir for two hours at room temperature and the volatiles were removed in vacuo. The crude, yellow solid was washed with $5 \mathrm{~mL}$ of cold ethyl ether and filtered to give $4 \mathbf{d}(1.27 \mathrm{~g}, 76.0 \%)$ as a bright yellow solid. Mp: $190-210{ }^{\circ} \mathrm{C}$ dec. ${ }^{1} \mathbf{H}$ NMR (200 MHz, DMSO-d 6 , ppm) $\delta 2.30(\mathrm{~s}, 6 \mathrm{H}, \mathrm{Me}), 5.64(\mathrm{t}, 1 \mathrm{H}, \mathrm{CHCHCH}), 6.18(\mathrm{~d}, 2 \mathrm{H}$, $\mathrm{CHCHCH}), 7.07$ (d, 4H, Ar), $7.56(\mathrm{~d}, 4 \mathrm{H}, \mathrm{Ar}) \cdot{ }^{13} \mathrm{C}\left\{{ }^{1} \mathrm{H}\right\}$ NMR (50 MHz, DMSO-d, ppm) $\delta 20.8(\mathrm{Me}), 109.6(\mathrm{CHCHCH}), 122.6(\mathrm{CHCHCH}), 125.4(\mathrm{CC}), 127.6,129.2,138.6$, 140.5 (Ar), 188.7 (p-TolCO). IR (KBr, cm $\left.{ }^{-1}\right) 3027$ (Me), 1606 (Ar), 1346 (C-O). MS (+El) $\mathrm{m} / \mathrm{z} 507\left(\mathrm{M}^{+}\right)$. Anal. Calcd for $\mathrm{C}_{21} \mathrm{H}_{18} \mathrm{O}_{2} \mathrm{TI}$ : C, 49.87; H, 3.39. Found: C, 49.97; $\mathrm{H}$, 3.28 .

Synthesis of $\left[\mathrm{TI}\left\{1,2-\mathrm{C}_{5} \mathrm{H}_{3}\left(\mathrm{CO}-4-\mathrm{MeOC}_{6} \mathrm{H}_{4}\right)_{2}\right\}\right](4 \mathrm{e})$. To a $125-\mathrm{mL}$ Schlenk flask, TIOEt (762 mg, $3.05 \mathrm{mmol})$ was added via syringe to a stirred solution of $1 \mathrm{e}(1.02 \mathrm{~g}$, $3.05 \mathrm{mmol}$ ) in $30 \mathrm{~mL}$ of THF. A yellow precipitate immediately formed. The suspension was allowed to stir for two hours at room temperature and the volatiles were removed in 
vacuo. The crude, yellow solid was washed with cold ethyl ether and filtered to give $4 \mathbf{e}$ (1.25 g, 76.2\%) as a bright yellow solid. Mp: $170-200{ }^{\circ} \mathrm{C}$ dec. ${ }^{1} \mathbf{H}$ NMR $(200 \mathrm{MHz}$, DMSO-d $_{6}$ ppm) $\delta 3.76(\mathrm{~s}, 6 \mathrm{H}, \mathrm{MeO}), 5.65(\mathrm{t}, 1 \mathrm{H}, \mathrm{J}=3.6 \mathrm{~Hz}, \mathrm{CHCHCH}), 6.19(\mathrm{~d}, 2 \mathrm{H}, \mathrm{J}=$ 3.6, $\mathrm{CHCHCH}), 6.83(\mathrm{~d}, 4 \mathrm{H}, \mathrm{J}=8.8, \mathrm{Ar}), 7.66(\mathrm{~d}, 4 \mathrm{H}, \mathrm{J}=8.8, \mathrm{Ar}) \cdot{ }^{13} \mathrm{C}\left\{{ }^{1} \mathrm{H}\right\}$ NMR $(50$ MHz, $\left.\mathrm{CDCl}_{3}, \mathbf{p p m}\right) \delta 55.0(\mathrm{MeO}), 109.4(\mathrm{CHCHCH}), 112.3(\mathrm{CHCHCH}), 121.9(\mathrm{CC})$, 125.4, 130.9, 135.7, 160.3, $188.2\left(\mathrm{COp}-\mathrm{MeOC}_{6} \mathrm{H}_{4}\right) . \mathbf{I R}\left(\mathrm{KBr}, \mathbf{c m}^{-1}\right) 1602$ (CO), 1255 (MeO). MS (+EI): m/z $538\left(\mathrm{M}^{+}\right)$.

Synthesis of $\left[\mathrm{TI}\left\{1,2-\mathrm{C}_{5} \mathrm{H}_{3}(\mathrm{COTp})_{2}\right\}\right]$ (4f). Thallium(I) ethoxide $(2.46 \mathrm{~g}, 9.86$ mmol) was added to a solution of 1,2- $\mathrm{C}_{5} \mathrm{H}_{3}(\mathrm{CTpOH})(\mathrm{COTp})(\mathbf{1 f}, 2.56 \mathrm{~g}, 9.02 \mathrm{mmol})$ in THF. A bright yellow solid precipitated immediately. The solution was stirred for 2 hours. The precipitate was filtered and washed with $5 \mathrm{~mL}$ of cold ethyl ether affording a quantitative yield of $\left[\mathrm{TI}\left\{1,2-\mathrm{C}_{5} \mathrm{H}_{3}(\mathrm{COTp})_{2}\right\}\right]$ (4f). Mp: $\sim 185-195{ }^{\circ} \mathrm{C}$ dec. ${ }^{\mathbf{1}} \mathbf{H}$ NMR (200 MHz, DMSO-d 6 , ppm) $\delta 5.68(\mathrm{t}, 1 \mathrm{H}, \mathrm{J}=3.3 \mathrm{~Hz}, \mathrm{CHCHCH}), 6.43(\mathrm{~d}, 2 \mathrm{H}, \mathrm{J}=3.3 \mathrm{~Hz}$, $\mathrm{CHCHCH}$ ), 7.03 (dd, 2H, J = 4.7, 3.7 Hz, Tp), $7.47(\mathrm{dd}, 2 \mathrm{H}, \mathrm{J}=3.7,0.7 \mathrm{~Hz}, \mathrm{Tp}), 7.60$ $(\mathrm{dd}, 2 \mathrm{H}, \mathrm{J}=4.7,0.7, \mathrm{Tp}) .{ }^{13} \mathrm{C}\left\{{ }^{1} \mathrm{H}\right\}$ NMR (200 MHz, DMSO-d 6, ppm) $\delta 110.1$ (CHCHCH), 122.3 (CHCHCH), 124.9 (CC), 127.0 (Tp), 129.6 (Tp), 130.1 (Tp), 149.0 (COCS), 180.5 (CO). IR (KBr, cm-1) 3080 (C-H), 1565 (CO), 1503, 1411, 1358. MS (+EI) $\mathrm{m} / \mathrm{z} 490\left(\mathrm{M}^{+}\right)$. Anal. Calcd for $\mathrm{C}_{15} \mathrm{H}_{9} \mathrm{O}_{2} \mathrm{~S}_{2} \mathrm{TI}$ : $\mathrm{C}, 36.79 ; \mathrm{H}, 1.85$; Found: $\mathrm{C}, 37.01$; $H, 1.99$.

Synthesis of $\left[\mathrm{TI}\left\{1,2-\mathrm{C}_{5} \mathrm{H}_{3}\left(\mathrm{CO}-2-\mathrm{C}_{4} \mathrm{H}_{2} \mathrm{~S}-5-\mathrm{Me}\right)_{2}\right\}\right]$ (4g). Thallium(I) ethoxide (0.434 g, $0.124 \mathrm{~mL}, 1.74 \mathrm{mmol})$ was added to a solution of $1,2-\mathrm{C}_{5} \mathrm{H}_{3}\left(\mathrm{CO}-2-\mathrm{C}_{4} \mathrm{H}_{2} \mathrm{~S}-5-\right.$ $\mathrm{Me})\left(\mathrm{COH}-2-\mathrm{C}_{4} \mathrm{H}_{2} \mathrm{~S}-5-\mathrm{Me}\right)(\mathbf{1 g}, 0.500 \mathrm{~g}, 1.59 \mathrm{mmol})$ in THF $(40 \mathrm{~mL})$. A bright yellow solid precipitated immediately. The solution was stirred for 2 hours at $22{ }^{\circ} \mathrm{C}$. The precipitate 
was filtered and washed with cold ethyl ether $(3 \times 5 \mathrm{~mL})$ providing $\left[T \mid\left\{1,2-\mathrm{C}_{5} \mathrm{H}_{3}(\mathrm{CO}-2-\right.\right.$ $\left.\left.\left.\mathrm{C}_{4} \mathrm{H}_{2} \mathrm{~S}-5-\mathrm{Me}\right)_{2}\right\}\right](\mathbf{4 g}, 0.816 \mathrm{~g}, 99.1 \%)$. dec. $200-201{ }^{\circ} \mathrm{C} .{ }^{1} \mathrm{H}$ NMR (200 MHz, DMSO, ppm) $\delta 2.44(\mathrm{~s}, 6 \mathrm{H}, \mathrm{Me}), 5.67\left(\mathrm{t},{ }^{3} \mathrm{~J}_{\mathrm{HH}}=3.30 \mathrm{~Hz}, 1 \mathrm{H}, \mathrm{CHCHCH}\right), 6.40\left(\mathrm{~d},{ }^{3} \mathrm{~J}_{\mathrm{HH}}=3.30\right.$ $\mathrm{Hz}, 2 \mathrm{H}, \mathrm{CHCHCH}), 6.75\left(\mathrm{~d},{ }^{3} \mathrm{~J}_{\mathrm{HH}}=3.66 \mathrm{~Hz}, 1 \mathrm{H}, \mathrm{CHCH}\right), 7.30\left(\mathrm{~d},{ }^{3} \mathrm{~J}_{\mathrm{HH}}=3.66 \mathrm{~Hz}, 1 \mathrm{H}\right.$, $\mathrm{CHCH}) .{ }^{13} \mathrm{C}\left\{{ }^{1} \mathrm{H}\right\}$ NMR (50 MHz, DMSO, ppm) $\delta 15.4(\mathrm{Me}), 109.8(\mathrm{CHCHCH}), 121.8$ (CHCHCH), 124.7 (CC), 125.7, 130.6, 143.6, $146.7(\mathrm{Tp}), 180.7(\mathrm{CO}) . \mathbf{I R}\left(\mathrm{KBr}, \mathbf{c m}^{-1}\right)$ 1548.3 (CO), $2962.7\left(\mathrm{CH}_{3}\right)$. MS (+EI): m/z $518\left(\mathrm{M}^{+}\right), 314\left(\mathrm{M}^{+}-\mathrm{TI}\right), 216\left(\mathrm{M}^{+}-\mathrm{TI}-\mathrm{C}_{4} \mathrm{H}_{2} \mathrm{SMe}\right)$, $119\left(\mathrm{M}^{+}-\mathrm{Tl}-2 \mathrm{C}_{4} \mathrm{H}_{2} \mathrm{SMe}\right)$. Anal. Calcd for $\mathrm{C}_{17} \mathrm{H}_{13} \mathrm{O}_{2} \mathrm{~S}_{2} \mathrm{Tl}$ : C, 37.81; $\mathrm{H}, 2.31$; Found: $\mathrm{C}$, 37.20; H 2.37 .

Synthesis of $\left[\mathrm{TI}\left\{1,2-\mathrm{C}_{5} \mathrm{H}_{3}\left(\mathrm{COC}_{8} \mathrm{H}_{5} \mathrm{~S}\right)_{2}\right\}\right]$ (4h). In a $125-\mathrm{mL}$ Schlenk flask, TIOEt (696 $\mathrm{mg}, 2.79 \mathrm{mmol})$ was added via syringe to a stirred solution of $\mathbf{1 h}(1.08 \mathrm{~g}, 2.79$ $\mathrm{mmol}$ ) in $30 \mathrm{~mL}$ of THF. A red-orange precipitate formed immediately. The suspension was allowed to stir for two hours at room temperature and the volatiles were removed in vacuo. The crude product was washed with cold ethyl ether and filtered to give $4 \mathrm{~h}$ (921 mg, 56.0\%) as a red-orange solid. Mp: $>230{ }^{\circ} \mathrm{C} .{ }^{1} \mathrm{H}$ NMR (200 MHz, DMSO-d 6 ppm) $\delta 5.80(\mathrm{t}, 1 \mathrm{H}, \mathrm{J}=3.6 \mathrm{~Hz}, \mathrm{CHCHCH}), 6.60(\mathrm{~d}, 2 \mathrm{H}, \mathrm{J}=3.6, \mathrm{CHCHCH}), 7.34-7.39(\mathrm{~m}, 6 \mathrm{H}$, Ar), 7.81 (s, 2H, Tp), 7.85-7.94 (m, 2H, Ar). ${ }^{13} \mathrm{C}\left\{{ }^{1} \mathrm{H}\right\}$ NMR (50 MHz, $\left.\mathrm{CDCl}_{3}, \mathrm{ppm}\right)$ $\delta 111.0(\mathrm{CHCHCH}), 122.6(\mathrm{CHCHCH}), 123.5(\mathrm{CC}), 124.3,124.9,125.2,125.4,126.6$, 139.5, 140.5 (Ar), 181.1 (CO). IR (KBr, cm $\left.{ }^{-1}\right) 1598$ (CO). MS (+EI) m/z $590\left(\mathrm{M}^{+}\right)$. Anal. Calcd for $\mathrm{C}_{23} \mathrm{H}_{13} \mathrm{~S}_{2} \mathrm{O}_{2} \mathrm{Tl}: \mathrm{C}, 46.81 ; \mathrm{H}, 2.22$. Found: $\mathrm{C}, 37.64 ; \mathrm{H}, 1.35$.

Synthesis of $\left[\mathrm{Mn}(\mathrm{CO})_{3}\left\{\eta^{5}-1,2-\mathrm{C}_{5} \mathrm{H}_{3}(\mathrm{COMe})_{2}\right\}\right]$ (5a). To a $100-\mathrm{mL}$ Schlenk flask, $\left[\mathrm{MnBr}(\mathrm{CO})_{5}\right](150 \mathrm{mg}, 0.546 \mathrm{mmol})$ was added to a stirred solution of $4 \mathrm{a}(192 \mathrm{mg}, 0.526$ $\mathrm{mmol}$ ) in $40 \mathrm{~mL}$ of benzene. The solution was allowed to reflux overnight. The reaction 
mixture was cooled and passed through a thin pad of Celite. The volatiles were removed in vacuo and trituration with cold pentane gave $\mathbf{5 a}(133 \mathrm{mg}, 83.6 \%)$ as a cream-colored powder. Slow recrystallization from ethyl ether at room temperature in air gave yellow, single crystals. Mp: $43-46{ }^{\circ} \mathrm{C} .{ }^{1} \mathbf{H}$ NMR (200 MHz, $\left.\mathbf{C}_{6} \mathbf{D}_{6}, \mathbf{p p m}\right) \delta 1.97(\mathrm{~s}$, $6 \mathrm{H}, \mathrm{Me}), 3.56(\mathrm{t}, 1 \mathrm{H}, \mathrm{J}=2.8 \mathrm{~Hz}, \mathrm{CHCHCH}), 4.34(\mathrm{~d}, 2 \mathrm{H}, \mathrm{J}=2.8 \mathrm{~Hz}, \mathrm{CHCHCH}) .{ }^{13} \mathrm{C}\left\{{ }^{1} \mathrm{H}\right\}$ NMR (50 MHz, $\left.\mathbf{C}_{6} \mathrm{D}_{6}, \mathbf{p p m}\right) \delta 28.4(\mathrm{Me}), 79.3(\mathrm{CHCHCH}), 87.7(\mathrm{CHCHCH}), 98.3(\mathrm{CC})$, 194.4 (COMe), 222.3 (CO). IR (KBr, cm ${ }^{-1}$ ) 2027, 1964, 1942 (CO), 1704, 1674, 1663 (COMe). MS (MALDI-TOF): m/z $460\left(\mathrm{M}^{+}+\right.$Matrix-Me), $389\left(\mathrm{M}^{+}+\right.$Matrix-2COMe). Anal. Calc for $\mathrm{C}_{12} \mathrm{H}_{9} \mathrm{O}_{5} \mathrm{Mn}: \mathrm{C}, 50.02 ; \mathrm{H}, 3.15$. Found: C, 49.95; $\mathrm{H}, 2.92$.

Synthesis of $\left[\mathrm{Mn}\left\{\eta^{5}-1,2-\mathrm{C}_{5} \mathrm{H}_{3}\left(\mathrm{CO}^{t} \mathrm{Bu}\right)_{2}\right\}(\mathrm{CO})_{3}\right](5 \mathrm{~b})$. To a suspension of $[\mathrm{TI}\{1,2-$ $\left.\left.\mathrm{C}_{5} \mathrm{H}_{3}\left(\mathrm{CO}^{\mathrm{t}} \mathrm{Bu}\right)_{2}\right\}\right](\mathbf{4 b}, 0.500 \mathrm{~g}, 1.14 \mathrm{mmol})$ in benzene $(40 \mathrm{~mL})$ was added $\left[\mathrm{MnBr}(\mathrm{CO})_{5}\right]$ (0.344 g, $1.25 \mathrm{mmol})$. The suspension was warmed to reflux and stirred overnight. The solution was filtered through Celite and the solvent removed in vacuo to give $\mathbf{5 b}(0.325$ g, $0.874 \mathrm{mmol}, 76.7 \%)$ as a yellow solid. Mp: $105-106{ }^{\circ} \mathrm{C} .{ }^{1} \mathrm{H}$ NMR (200 MHz, DMSO, ppm): $\delta 1.14\left(\mathrm{~s}, 9 \mathrm{H},{ }^{\mathrm{t}} \mathrm{Bu}\right), 4.97\left(\mathrm{t},{ }^{3} \mathrm{~J}_{\mathrm{HH}}=2.80 \mathrm{~Hz}, 1 \mathrm{H}, \mathrm{CHCHCH}\right), 5.85\left(\mathrm{~d},{ }^{3} \mathrm{~J}_{\mathrm{HH}}=2.80\right.$ $\mathrm{Hz}, 2 \mathrm{H}, \mathrm{CHCHCH}) .{ }^{13} \mathrm{C}$ NMR (50 MHz, DMSO, ppm): $\delta 27.1\left(\mathrm{CCH}_{3}\right), 43.8\left(\mathrm{CCH}_{3}\right), 78.7$ ( $\mathrm{CHCHCH}), 87.8(\mathrm{CHCHCH}), 101.7(\mathrm{CC}), 204.5\left(\mathrm{CO}^{\mathrm{t}} \mathrm{Bu}\right), 223.3(\mathrm{MnCO}) . \mathbf{I R}\left(\mathrm{KBr}, \mathbf{~ c m}^{-}\right.$ $\left.{ }^{1}\right):$ 1692, 1659 (CO'Bu), 2031, 1960, 1933 (MnCO), 3141, 3096, $3037(\mathrm{Ar}-\mathrm{H}), 2967$, 2935, $2872\left(\mathrm{CH}_{3}\right)$. MS (+EI): m/z $372\left(\mathrm{M}^{+}\right), 232\left(\mathrm{M}^{+}-\mathrm{Mn}(\mathrm{CO})_{3}\right)$. Anal. Calcd for $\mathrm{C}_{18} \mathrm{H}_{21} \mathrm{O}_{5} \mathrm{Mn}: \mathrm{C}, 58.10 ; \mathrm{H}, 5.65$; Found: $\mathrm{C}, 53.51 ; 2.90 \mathrm{H}$.

Synthesis of $\left[\mathrm{Mn}\left\{\eta^{5}-1,2-\mathrm{C}_{5} \mathrm{H}_{3}(\mathrm{COPh})_{2}\right\}(\mathrm{CO})_{3}\right](5 \mathrm{c})$. To a suspension of $[\mathrm{TI}\{1,2-$ $\left.\left.\mathrm{C}_{5} \mathrm{H}_{3}(\mathrm{COPh})_{2}\right\}\right](4 \mathrm{c}, 0.500 \mathrm{~g}, 1.05 \mathrm{mmol})$ in benzene $(40 \mathrm{~mL})$ was added $\left[\mathrm{MnBr}(\mathrm{CO})_{5}\right]$ $(0.316 \mathrm{~g}, 1.15 \mathrm{mmol})$. The suspension was warmed to reflux and stirred for $4.5 \mathrm{hr}$. The 
solution was filtered through Celite and the solvent removed in vacuo to give [Mn\{ $\left\{\eta^{5}-\right.$ 1,2- $\left.\left.\mathrm{C}_{5} \mathrm{H}_{3}(\mathrm{COPh})_{2}\right\}(\mathrm{CO})_{3}\right](5 \mathrm{c}, 0.273 \mathrm{~g}, 0.845 \mathrm{mmol}, 63.0 \%) . \mathbf{M p :} 162-164{ }^{\circ} \mathrm{C} .{ }^{1} \mathbf{H}$ NMR (200 MHz, DMSO, ppm) $\delta 5.21\left(\mathrm{t},{ }^{3} \mathrm{~J}_{\mathrm{HH}}=2.93 \mathrm{~Hz}, 1 \mathrm{H}, \mathrm{CHCHCH}\right), 5.87\left(\mathrm{~d},{ }^{3} \mathrm{~J}_{\mathrm{HH}}=2.93\right.$ $\mathrm{Hz}, 2 \mathrm{H}, \mathrm{CHCHCH}), 7.45-7.84(\mathrm{~m}, 10 \mathrm{H}, \mathrm{Ph}) .{ }^{13} \mathrm{C}\left\{{ }^{1} \mathrm{H}\right\}$ NMR (50 MHz, DMSO, ppm) $\delta$ $80.8(\mathrm{CHCHCH}), 89.8(\mathrm{CHCHCH}), 101.7(\mathrm{CC}), 128.5,128.7,133.4,136.4(\mathrm{Ph}), 190.3$ (COPh), 223.0 (MnCO). IR (KBr, cm ${ }^{-1}$ ) 2025, 1956, 1943 (CO), 3114, 3072 (C-H). IR (Nujol, cm ${ }^{-1}$ ) 2025, 1957, 1943 (CO), 3144, 3090 (C-H). MS (+El): m/z $413\left(\right.$ M $\left.^{+}\right), 383$ $\left(\mathrm{M}^{+}-\mathrm{CO}\right), 328\left(\mathrm{M}^{+}-3 \mathrm{CO}\right), 274 \quad\left(\mathrm{M}^{+}-\mathrm{Mn}(\mathrm{CO})_{3}\right), 202 \quad\left(\mathrm{M}^{+}-2 \mathrm{COPh}\right), 169 \quad\left(\mathrm{M}^{+}-\mathrm{COPh}-\right.$ $\left.\mathrm{Mn}(\mathrm{CO})_{3}\right)$. Anal. Calcd for $\mathrm{C}_{22} \mathrm{H}_{13} \mathrm{MnO}_{5}$ : C, 64.09; H, 3.18; Found: C, 63.82, H, 2.96 .

Synthesis of $\left[\mathrm{Mn}(\mathrm{CO})_{3}\left\{\eta^{5}-1,2-\mathrm{C}_{5} \mathrm{H}_{3}(\mathrm{CO}-4-\mathrm{Tol})_{2}\right\}\right](5 \mathrm{~d})$. To a $100-\mathrm{mL}$ Schlenk flask, $\left[\mathrm{MnBr}(\mathrm{CO})_{5}\right](250 \mathrm{mg}, 0.910 \mathrm{mmol})$ was added to a stirred solution of $4 \mathrm{~d}(460 \mathrm{mg}$, $0.910 \mathrm{mmol}$ ) in $40 \mathrm{~mL}$ of benzene. The solution was allowed to reflux overnight. The reaction mixture was cooled and passed through a thin pad of Celite and the volatiles were removed in vacuo. The crude product was triturated with cold pentane to yield $\mathbf{5 d}$ (366 mg, 91.4\%) as a light yellow solid. An analytically pure sample was obtained by recrystallization from hot hexanes. Mp: $113-115{ }^{\circ} \mathrm{C} .{ }^{1} \mathbf{H}$ NMR $\left(200 \mathrm{MHz}, \mathrm{C}_{6} \mathrm{D}_{6}, \mathrm{ppm}\right)$ $\delta 1.86(\mathrm{~s}, 6 \mathrm{H}, \mathrm{Me}), 3.72(\mathrm{t}, 1 \mathrm{H}, \mathrm{J}=2.8 \mathrm{~Hz}, \mathrm{CHCHCH}), 4.62(\mathrm{~d}, 2 \mathrm{H}, \mathrm{J}=2.8 \mathrm{~Hz}$, $\mathrm{CHCHCH}), 6.74(\mathrm{~d}, 4 \mathrm{H}, \mathrm{J}=8.0 \mathrm{~Hz}, \operatorname{Ar}), 7.65(\mathrm{~d}, 4 \mathrm{H}, \mathrm{J}=8.0 \mathrm{~Hz}, \operatorname{Ar}) \cdot{ }^{13} \mathrm{C}\left\{{ }^{1} \mathrm{H}\right\}$ NMR (50 MHz, $\left.\mathbf{C}_{6} \mathbf{D}_{6}, \mathbf{p p m}\right) \delta 20.9(\mathrm{Me}), 78.6(\mathrm{CHCHCH}), 87.3(\mathrm{CHCHCH}), 102.5(\mathrm{CC}), 128.7$, 128.8, 134.8, 143.2 (Ar), 188.9 (p-TolCO), 222.5 (CO). IR (KBr, cm ${ }^{-1}$ ) 2026, 1963, 1943 (CO). MS (+EI) m/z $440\left(\mathrm{M}^{+}\right)$. Anal. Calcd for $\mathrm{C}_{24} \mathrm{H}_{17} \mathrm{O}_{5} \mathrm{Mn}$ : C, 65.46; H, 3.89. Found: $\mathrm{C}$, 65.48; H, 3.83 . 
Synthesis of $\left[\mathrm{Mn}(\mathrm{CO})_{3}\left\{\eta^{5}-1,2-\mathrm{C}_{5} \mathrm{H}_{3}\left(\mathrm{CO}-4-\mathrm{MeOC}_{6} \mathrm{H}_{4}\right)_{2}\right\}\right]$ (5e). To a $125-\mathrm{mL}$ Schlenk flask, $\left[\mathrm{MnBr}(\mathrm{CO})_{5}\right](177 \mathrm{mg}, 0.546 \mathrm{mmol})$ was added to a stirred solution of $4 \mathrm{e}$ (346 mg, $0.643 \mathrm{mmol}$ ) in $40 \mathrm{~mL}$ of benzene. The solution was allowed to reflux overnight. The reaction mixture was cooled and passed through a thin pad of Celite and the volatiles were removed in vacuo. The crude product was recrystallized in a ethyl ether/hexanes mixture to yield $\mathbf{5 e}(175 \mathrm{mg}, 54.7 \%)$ as a light yellow solid. Slow recrystallization from ethyl ether at room temperature in air gave single, yellow crystals. Mp: $116-118{ }^{\circ} \mathrm{C} .{ }^{1} \mathrm{H}$ NMR $\left(200 \mathrm{MHz}, \mathrm{C}_{6} \mathrm{D}_{6}, \mathrm{ppm}\right) \delta 3.05(\mathrm{~s}, 6 \mathrm{H}, \mathrm{OMe}), 3.76(\mathrm{t}, 1 \mathrm{H}, \mathrm{J}=$ $2.8 \mathrm{~Hz}, \mathrm{CHCHCH}), 4.63(\mathrm{~d}, 2 \mathrm{H}, \mathrm{J}=2.8 \mathrm{~Hz}, \mathrm{CHCHCH}), 6.48\left(\mathrm{dt}, 4 \mathrm{H},{ }^{1} \mathrm{~J}=7 \mathrm{~Hz},{ }^{2} \mathrm{~J}=1.8\right.$ $\mathrm{Hz}, m-\mathrm{Ar}), 7.74\left(\mathrm{dt}, 4 \mathrm{H},{ }^{1} \mathrm{~J}=7 \mathrm{~Hz},{ }^{2} \mathrm{~J}=1.8 \mathrm{~Hz}, \mathrm{o}-\mathrm{Ar}\right) .{ }^{13} \mathrm{C}\left\{{ }^{1} \mathrm{H}\right\} \mathrm{NMR}\left(50 \mathrm{MHz}, \mathrm{C}_{6} \mathrm{D}_{6}\right.$, ppm) $\delta 55.1(\mathrm{MeO}), 79.4(\mathrm{CHCHCH}), 87.5(\mathrm{CHCHCH}), 103.9(\mathrm{CC}), 114.3(m-\mathrm{Ar}), 131.0$ (ipso-CCO), 131.7 (o-Ar), 164.1 (ipso-COMe), 188.6 (COp-MeOC $\left.{ }_{6} \mathrm{H}_{4}\right), 223.6$ (CO). IR $\left(\mathrm{KBr}, \mathbf{c m}^{-1}\right)$ 2027, 1952, 1931 (CO). MS (El) m/z $473\left(\mathrm{M}^{+}+\mathrm{H}\right)$. Anal. Calcd for $\mathrm{C}_{24} \mathrm{H}_{17} \mathrm{O}_{7} \mathrm{Mn}: \mathrm{C}, 61.03 ; \mathrm{H}, 3.63$. Found: $\mathrm{C}, 61.00 ; \mathrm{H}, 3.43$.

Synthesis of $\left[\mathrm{Mn}\left\{\eta^{5}-1,2-\mathrm{C}_{5} \mathrm{H}_{3}(\mathrm{COTp})_{2}\right\}(\mathrm{CO})_{3}\right](5 f)$. To a suspension of $[\mathrm{TI}\{1,2-$ $\left.\left.\mathrm{C}_{5} \mathrm{H}_{3}(\mathrm{COTp})_{2}\right\}\right](\mathbf{2 f}, 0.500 \mathrm{~g}, 1.02 \mathrm{mmol})$ in benzene $(40 \mathrm{~mL})$ was added $\left[\mathrm{MnBr}(\mathrm{CO})_{5}\right]$ $(0.309 \mathrm{~g}, 1.12 \mathrm{mmol})$. The suspension was warmed to reflux and stirred for $4.5 \mathrm{hr}$. The solution was filtered through Celite and the solvent removed in vacuo to give $\left[\mathrm{Mn}\left\{\eta^{5}-\right.\right.$ 1,2- $\left.\left.\mathrm{C}_{5} \mathrm{H}_{3}(\mathrm{COTp})_{2}\right\}(\mathrm{CO})_{3}\right](\mathbf{5 f}, 0.324 \mathrm{~g}, 0.764 \mathrm{mmol}, 75.0 \%)$. Mp: $158-159{ }^{\circ} \mathrm{C} .{ }^{1} \mathbf{H}$ NMR (200 MHz, DMSO, ppm) $\delta 5.26\left(\mathrm{t},{ }^{3} \mathrm{~J}_{\mathrm{HH}}=2.56 \mathrm{~Hz}, 1 \mathrm{H}, \mathrm{CHCHCH}\right), 6.08\left(\mathrm{~d},{ }^{3} \mathrm{~J}_{\mathrm{HH}}=2.56\right.$ $\mathrm{Hz}, 2 \mathrm{H}, \mathrm{CHCHCH}), 7.24\left(\mathrm{t},{ }^{3} \mathrm{~J}_{\mathrm{HH}}=4.76 \mathrm{~Hz}, 1 \mathrm{H}, \mathrm{SCHCHCH}\right), 7.83\left(\mathrm{~d},{ }^{3} \mathrm{~J}_{\mathrm{HH}}=3.66 \mathrm{~Hz}\right.$, $1 \mathrm{H}, \mathrm{SCHCHCH}), 8.08\left(\mathrm{~d},{ }^{3} \mathrm{~J}_{\mathrm{HH}}=5.13 \mathrm{~Hz}, 1 \mathrm{H}, \mathrm{SCHCHCH}\right) .{ }^{13} \mathrm{C}\left\{{ }^{1} \mathrm{H}\right\} \mathrm{NMR}(50 \mathrm{MHz}$, DMSO, ppm) $\delta 80.2(\mathrm{CHCHCH}), 89.1(\mathrm{CHCHCH}), 101.2(\mathrm{CC}), 128.9,134.4,136.0$, 
142.3 (Tp), 181.4 (COTp), 223.0 (MnCO). IR (KBr, cm ${ }^{-1}$ ) 1662 (COTp), 2040, 1964 (MnCO), 3063 (C-H). IR (Nujol, cm ${ }^{-1}$ ) 1645 (COTp), 2028, 1955, 1927 (MnCO), 3108, $3091(\mathrm{C}-\mathrm{H})$. MS: m/z $424\left(\mathrm{M}^{+}\right), 395\left(\mathrm{M}^{+}-\mathrm{CO}\right), 368\left(\mathrm{M}^{+}-2 \mathrm{CO}\right), 340\left(\mathrm{M}^{+}-3 \mathrm{CO}\right), 286\left(\mathrm{M}^{+}-\right.$ $\left.\mathrm{Mn}(\mathrm{CO})_{3}\right), 174\left(\mathrm{M}^{+}-\mathrm{SC}_{4} \mathrm{H}_{3}-\mathrm{Mn}(\mathrm{CO})_{3}\right)$. Anal. Calcd for $\mathrm{C}_{18} \mathrm{H}_{9} \mathrm{MnO}_{5}: \mathrm{C}, 50.95 ; \mathrm{H}, 2.14$; Found: C, 51.07, H, 2.09.

\section{Synthesis of $\left[\mathrm{Mn}\left\{\eta^{5}-1,2-\mathrm{C}_{5} \mathrm{H}_{3}\left(\mathrm{CO}-2-\mathrm{C}_{4} \mathrm{H}_{2} \mathrm{~S}-5-\mathrm{Me}\right)_{2}\right\}(\mathrm{CO})_{3}\right](5 \mathrm{~g})$. To a} suspension of $\left[\mathrm{TI}\left\{1,2-\mathrm{C}_{5} \mathrm{H}_{3}\left(\mathrm{CO}-2-\mathrm{C}_{4} \mathrm{H}_{2} \mathrm{~S}-5-\mathrm{Me}\right)_{2}\right\}\right](\mathbf{4 g}, 0.500 \mathrm{~g}, 0.965 \mathrm{mmol})$ in benzene $(40 \mathrm{~mL})$ was added $\left[\mathrm{MnBr}(\mathrm{CO})_{5}\right](0.292 \mathrm{~g}, 1.06 \mathrm{mmol})$. The suspension was warmed to reflux and stirred overnight. The solution was filtered through Celite and the solvent removed in vacuo to give $5 \mathrm{~g}(0.382 \mathrm{~g}, 0.845 \mathrm{mmol}, 87.6 \%)$ as a yellow solid. Mp: $168-169{ }^{\circ} \mathrm{C} .{ }^{1} \mathrm{H}$ NMR $(200 \mathrm{MHz}$, DMSO, ppm) $\delta 2.38$ (s, $3 \mathrm{H}, \mathrm{Me}), 5.03\left(\mathrm{t},{ }^{3} \mathrm{~J}_{\mathrm{HH}}=2.56 \mathrm{~Hz}, 1 \mathrm{H}, \mathrm{CHCHCH}\right), 5.87\left(\mathrm{~d},{ }^{3} \mathrm{~J}_{\mathrm{HH}}=2.56 \mathrm{~Hz}, 2 \mathrm{H}\right.$, $\mathrm{CHCHCH}), 6.83\left(\mathrm{~d},{ }^{3} \mathrm{~J}_{\mathrm{HH}}=3.66 \mathrm{~Hz}, 1 \mathrm{H}, \mathrm{CHCH}\right), 7.50\left(\mathrm{~d},{ }^{3} J_{\mathrm{HH}}=3.66 \mathrm{~Hz}, 1 \mathrm{H}\right.$, $\mathrm{CHCH}) .{ }^{13} \mathrm{C}\left\{{ }^{1} \mathrm{H}\right\}$ NMR (50 MHz, DMSO, ppm) $\delta 15.5(\mathrm{Me}), 80.2(\mathrm{CHCHCH}), 88.7$ (CHCHCH), 101.5 (CC), 127.8, 135.1, 142.3, 150.1 (Tp), 180.8 (COTp), 223.2 (MnCO). ${ }^{13} \mathrm{C}\left\{{ }^{1} \mathrm{H}\right\}$ NMR (50 MHz, $\left.\mathbf{C}_{6} \mathrm{D}_{6}, \mathbf{p p m}\right) \delta 15.7(\mathrm{Me}), 79.3(\mathrm{CHCHCH}), 87.1$ (CHCHCH), 102.9 (CC), 127.3, 134.3, 142.0, 150.9 (Tp), 181.0 (COTp), 223.4 (MnCO). IR (KBr, cm ${ }^{-1}$ ) 1657 (COTpMe), 2024, 1939 (MnCO), 3145, 3090 (CH), $2922\left(\mathrm{CH}_{3}\right.$ ). IR (Nujol, cm ${ }^{-1}$ ) 2029, 2012, 1955 (CO), 3144, 3090 (C-H). MS: m/z $453\left(\mathrm{M}^{+}\right), 368\left(\mathrm{M}^{+}-3 \mathrm{CO}\right), 314\left(\mathrm{M}^{+}-\mathrm{Mn}(\mathrm{CO})_{3}\right)$. Anal. Calcd for $\mathrm{C}_{20} \mathrm{H}_{13} \mathrm{O}_{5} \mathrm{~S}_{2} \mathrm{Mn}$ : C, 53.11; H, 2.90; Found: C, 53.51; $2.90 \mathrm{H}$.

Synthesis of $\left[\mathrm{Mn}(\mathrm{CO})_{3}\left\{\eta^{5}-1,2-\mathrm{C}_{5} \mathrm{H}_{3}\left(\mathrm{COC}_{8} \mathrm{H}_{5} \mathrm{~S}\right)_{2}\right\}\right]$ (5h). In a 125-mL Schlenk flask, $\left[\mathrm{MnBr}(\mathrm{CO})_{5}\right](150 \mathrm{mg}, 0.546 \mathrm{mmol})$ was added to a stirred solution 
of $4 \mathrm{~h}$ (316 $\mathrm{mg}, 0.546 \mathrm{mmol}$ ) in $40 \mathrm{~mL}$ of benzene. The solution was allowed to reflux overnight. The reaction mixture was cooled and passed through a thin pad of Celite and the volatiles were removed in vacuo. The crude product was triturated with cold pentane to give $5 \mathrm{~h}$ (244 $\mathrm{mg}, 88.5 \%$ ) as a yellow-orange solid. Mp: $112-114{ }^{\circ} \mathrm{C} .{ }^{1} \mathrm{H}$ NMR $\left(200 \mathrm{MHz}, \mathrm{C}_{6} \mathrm{D}_{6}, \mathrm{ppm}\right) \delta 3.74(\mathrm{t}, 1 \mathrm{H}, \mathrm{J}=2.6 \mathrm{~Hz}$, $\mathrm{CHCHCH}), 4.72(\mathrm{~d}, 2 \mathrm{H}, \mathrm{J}=2.6 \mathrm{~Hz}, \mathrm{CHCHCH}), 6.92-6.96(\mathrm{~m}, 6 \mathrm{H}, \mathrm{Ar}), 7.35-7.38$ $(\mathrm{m}, 2 \mathrm{H}, \mathrm{Ar}) 7.64$ (s, 2H, Tp). $\left.{ }^{13} \mathrm{C}^{1}{ }^{1} \mathrm{H}\right\}$ NMR $\left(50 \mathrm{MHz}, \mathrm{C}_{6} \mathrm{D}_{6}, \mathrm{ppm}\right) \delta 79.2$ $(\mathrm{CHCHCH}), 87.9(\mathrm{CHCHCH}), 102.2(\mathrm{CC}), 123.4,125.5,126.4,131.0,139.4$, 143.2, 143.3 (Ar), 183.0 (COBzTp), 222.9 (CO). IR (KBr, cm $\left.{ }^{-1}\right)$ 2032, 2008, 1943 (CO). MS (EI) m/z $506\left(\mathrm{M}^{+}-\mathrm{H}_{2} \mathrm{O}\right)$. Anal. Calcd for $\mathrm{C}_{26} \mathrm{H}_{13} \mathrm{~S}_{2} \mathrm{O}_{5} \mathrm{Mn}: \mathrm{C}, 59.55 ; \mathrm{H}$, 2.50. Found: C, 59.47; H, 2.54 .

Synthesis of $\left[\mathrm{Mn}\left\{\eta^{5}-\mathrm{SC}_{7} \mathrm{H}_{3}-1,3-\mathrm{Me}_{2}\right\}(\mathrm{CO})_{3}\right]$ (3a). 1,3-Dimethyl-4Hcyclopenta[c]thiophene $(2 \mathrm{a}, 0.26 \mathrm{~g}, 1.7 \mathrm{mmol})$ was charged to a Schlenk flask under nitrogen. Ethyl ether $(20 \mathrm{~mL})$ was added via cannula and $\mathrm{n}$-butyllithium $(0.80 \mathrm{~mL}, 1.8 \mathrm{mmol})$ was added via syringe at room temperature. The pale yellow solution turned red-orange and the reaction was stirred for 45 minutes at room temperature. $\mathrm{Me}_{3} \mathrm{SnCl}(0.37 \mathrm{~g}, 1.9 \mathrm{mmol})$ was added against a nitrogen counterflow. A white precipitate formed immediately upon addition. The reaction was stirred for an additional 45 minutes at room temperature. [ $\left.\mathrm{MnBr}(\mathrm{CO})_{5}\right](0.47 \mathrm{~g}$, $1.7 \mathrm{mmol}$ ). The reaction mixture was stirred at room temperature for 24 hours. The solution was filtered through a short plug of Celite with ethyl ether eluent. The solvent was removed in vacuo to leave an orange-red solid. Recrystallization from hot pentane gave orange, thin-plate crystals of $3 \mathrm{a}(0.47 \mathrm{~g}, 1.6 \mathrm{mmol}, 94 \%)$. 
Mp: 84-87 ${ }^{\circ} \mathrm{C}$. IR (KBr, $\mathbf{c m}^{-1}$ ): 2011, 1917 (CO); (thin film, $\left.\mathbf{c m}^{-1}\right)$ : 2016 (CO), 1929 (CO), 1694 (C=C); (Nujol, cm ${ }^{-1}$ ): 2023, 1938 (CO). 1H NMR (200 MHz, $\left.\mathrm{CDCl}_{3}, \mathrm{ppm}\right) \delta 5.28\left(\mathrm{t}, 1 \mathrm{H}, \mathrm{CHCHCH},{ }^{3} \mathrm{~J}_{\mathrm{HH}}=3.0 \mathrm{~Hz}\right), 5.09\left(\mathrm{~d}, 2 \mathrm{H}, \mathrm{CHCHCH},{ }^{3} \mathrm{~J}_{\mathrm{HH}}\right.$ $=3.0 \mathrm{~Hz}), 2.51(\mathrm{~s}, 3 \mathrm{H}, \mathrm{Me}) .{ }^{1} \mathrm{H}$ NMR $\left(200 \mathrm{MHz}, \mathbf{C}_{6} \mathbf{D}_{6}, \mathbf{p p m}\right) \delta 4.66(\mathrm{t}, 1 \mathrm{H}$, $\left.\mathrm{CHCHCH},{ }^{3} \mathrm{~J}_{\mathrm{HH}}=2.6 \mathrm{~Hz}\right), 4.48\left(\mathrm{~d}, 2 \mathrm{H}, \mathrm{CHCHCH},{ }^{3} \mathrm{~J}_{\mathrm{HH}}=2.6 \mathrm{~Hz}\right), 2.07(\mathrm{~s}, 3 \mathrm{H}$, Me). ${ }^{1} \mathrm{H}$ NMR (200 MHz, $\left.\left(\mathrm{CD}_{3}\right)_{2} \mathrm{CO}, \mathrm{ppm}\right) \delta 5.51$ (t, $1 \mathrm{H}, \mathrm{CHCHCH},{ }^{3} \mathrm{~J}_{\mathrm{HH}}=3.0$ $\mathrm{Hz}), 5.40\left(\mathrm{~d}, 2 \mathrm{H}, \mathrm{CHCHCH},{ }^{3} \mathrm{~J}_{\mathrm{HH}}=3.0 \mathrm{~Hz}\right), 2.55$ (s, 3H, Me). ${ }^{1} \mathrm{H}$ NMR $(200 \mathrm{MHz}$, $\left.\mathrm{CD}_{2} \mathrm{Cl}_{2}, \mathrm{ppm}\right) \delta 5.32(\mathrm{~b}, 1 \mathrm{H}, \mathrm{CHCHCH}), 5.16(\mathrm{~b}, 2 \mathrm{H}, \mathrm{CHCHCH}), 2.53$ (bs, 3H, Me). ${ }^{13} \mathrm{C}\left\{{ }^{1} \mathrm{H}\right\}$ NMR $\left(100 \mathrm{MHz},\left(\mathrm{CD}_{3}\right)_{2} \mathrm{CO}, \mathrm{ppm}\right) \delta 225.75(\mathrm{~b}, \mathrm{CO}), 123.20$ $\left(\mathrm{CH}_{3} \mathrm{CSC}\right), 116.53$ (CHCCS), $96.47(\mathrm{CHCHCH}), 63.72(\mathrm{CHCHCH}), 13.29(\mathrm{Me})$. ${ }^{13} \mathrm{C}$ NMR (100 MHz, $\left.\left(\mathrm{CD}_{3}\right)_{2} \mathrm{CO}, \mathrm{ppm}\right) \delta 225.74$ (b, CO), $123.20\left(\mathrm{~s}, \mathrm{CH}_{3} \mathrm{CSC}\right)$, $116.53(\mathrm{~s}, \mathrm{CHCCS}), 96.47\left(\mathrm{dt}, \mathrm{Cp},{ }^{1} \mathrm{~J}_{\mathrm{CH}}=176.07 \mathrm{~Hz},{ }^{2} \mathrm{~J}_{\mathrm{CH}}=5.89 \mathrm{~Hz}\right), 63.72$ (dt, $\left.\mathrm{Cp},{ }^{1} \mathrm{~J}_{\mathrm{CH}}=182.71 \mathrm{~Hz},{ }^{2} \mathrm{~J}_{\mathrm{CH}}=5.89 \mathrm{~Hz}\right), 13.29\left(\mathrm{q}, \mathrm{Me},{ }^{1} \mathrm{~J}_{\mathrm{CH}}=129.66 \mathrm{~Hz}\right) . \mathrm{MS}(\mathrm{EI}-$ 12eV, RT to $\left.100{ }^{\circ} \mathrm{C}\right) \mathrm{m} / \mathrm{z} 288\left(\mathrm{M}^{+}\right)$. Anal. Calcd for $\mathrm{C}_{12} \mathrm{H}_{9} \mathrm{O}_{3} \mathrm{SMn}: \mathrm{C}, 50.01 ; \mathrm{H}$ 3.15. Found C, 48.35; H 3.07. TGA $\left(\mathrm{N}_{2}, 20^{\circ} \mathrm{C} / \mathrm{min}\right.$ to $\left.525^{\circ} \mathrm{C}\right)$ Mass loss $40 \%$ $\left(70-225{ }^{\circ} \mathrm{C},-\mathrm{C}_{9} \mathrm{H}_{9}\right), 29 \%\left(225-350{ }^{\circ} \mathrm{C},-3 \mathrm{CO}\right), 7 \%\left(350-525{ }^{\circ} \mathrm{C},-\mathrm{S}+\mathrm{O}\right.$ ?), residue $24 \%(\mathrm{MnO})$.

Synthesis of $\left[\mathrm{Mn}\left\{\eta^{5}-\mathrm{SC}_{7} \mathrm{H}_{3}-1,3-\mathrm{Ph}_{2}\right\}(\mathrm{CO})_{3}\right](3 \mathrm{c})$. Method A. To a solution of n-butyllithium $(2.50 \mathrm{M}, 0.100 \mathrm{~mL}, 0.240 \mathrm{mmol})$ in ethyl ether $(20 \mathrm{~mL})$ was added 1,3-diphenyl-4H-cyclopenta[c]thiophene $(2 \mathrm{c}, 61.7 \mathrm{mg}, 0.226 \mathrm{mmol})$. The solution immediately turned purple and was allowed to stir for 10 minutes. $\mathrm{Me}_{3} \mathrm{SnCl}(49.0 \mathrm{mg}, 0.253 \mathrm{mmol})$ was added against a nitrogen counterflow and 
the solution turned a pale yellow. The reaction mixture was stirred for 45 minutes at room temperature. $\left[\mathrm{MnBr}(\mathrm{CO})_{5}\right](62.4 \mathrm{mg}, 0.226 \mathrm{mmol})$ was added to the reaction mixture against a nitrogen counterflow. The reaction mixture was stirred for 24 hours turning cherry red. The solution was filtered through a short plug of Celite with ethyl ether eluent. The ethyl ether was removed in vacuo to leave a red solid $\mathbf{3 c}$ in quantitative yield. The solid was recrystallized from hot pentane giving red, thin-plate crystals in $90 \%$ isolated yield. Method B. In a $125-\mathrm{mL}$ Schlenk flask, 5c (130 mg, $0.315 \mathrm{mmol})$ was added to a stirred suspension of $\mathrm{P}_{4} \mathrm{~S}_{10}(700 \mathrm{mg}, 1.58 \mathrm{mmol})$ and $\mathrm{NaHCO}_{3}(146 \mathrm{mg}, 1.73 \mathrm{mmol})$ in $40 \mathrm{~mL}$ of $\mathrm{CS}_{2}$. The reaction mixture was allowed to reflux for 2 hours and cooled to room temperature. The reaction mixture was then passed through a thin pad of alumina and the volatiles were removed in vacuo. Trituration with cold pentane gave $3 c$ (46 mg, 35.4\%) as a red powder. Slow recrystallization from ethyl ether at room temperature under nitrogen atmosphere gave red, single crystals. Mp: 154-157 ${ }^{\circ} \mathrm{C}$. ${ }^{1} \mathrm{H}$ NMR (200 MHz, DMSO, ppm) $\delta 5.90\left(\mathrm{t},{ }^{3} \mathrm{~J}_{\mathrm{HH}}=2.93 \mathrm{~Hz}, 1 \mathrm{H}\right.$, $\mathrm{CHCHCH}), 6.05\left(\mathrm{~d},{ }^{3} \mathrm{~J}_{\mathrm{HH}}=2.93 \mathrm{~Hz}, 2 \mathrm{H}, \mathrm{CHCHCH}\right), 7.30-7.38\left(\mathrm{~m}, 2 \mathrm{H}, H_{\text {para }}\right)$, 7.48-7.55 (m, 4H, $\left.H_{\text {meta }}\right), 7.79-7.83\left(\mathrm{~m}, 4 \mathrm{H}, H_{\text {ortho }}\right) .{ }^{1} \mathrm{H}$ NMR (200 MHz, $\mathrm{CDCl}_{3}$, ppm) $\delta 5.47\left(\mathrm{t},{ }^{3} \mathrm{~J}_{\mathrm{HH}}=2.6 \mathrm{~Hz}, 1 \mathrm{H}, \mathrm{CHCHCH}\right), 5.51\left(\mathrm{~d},{ }^{3} \mathrm{~J}_{\mathrm{HH}}=2.6 \mathrm{~Hz}, 2 \mathrm{H}\right.$, $\mathrm{CHCHCH}), 7.28-7.34\left(\mathrm{~m}, 2 \mathrm{H}, H_{\text {para }}\right), 7.41-7.48\left(\mathrm{~m}, 4 \mathrm{H}, H_{\text {meta }}\right), 7.67-7.70(\mathrm{~m}, 4 \mathrm{H}$, $H_{\text {ortho). }}{ }^{1} \mathrm{H}$ NMR (200 MHz, $\left.\mathrm{CD}_{2} \mathrm{Cl}_{2}, \mathrm{ppm}\right) \delta 5.07$ (t, ${ }^{3} \mathrm{~J}_{\mathrm{HH}}=1.10 \mathrm{~Hz}, 1 \mathrm{H}$, $\mathrm{CHCHCH}), 5.31\left(\mathrm{~d}, 2 \mathrm{H},{ }^{3} \mathrm{~J}_{\mathrm{HH}}=1.10 \mathrm{~Hz}, \mathrm{CHCHCH}\right), 7.03-7.11\left(\mathrm{~m}, 2 \mathrm{H}, H_{\text {para }}\right)$, 7.20-7.28 (m, 4H, $\left.H_{\text {meta }}\right), 7.45-7.50\left(\mathrm{~m}, 4 \mathrm{H}, H_{\text {ortho }}\right) .{ }^{13} \mathrm{C}\left\{{ }^{1} \mathrm{H}\right\}$ NMR $(50 \mathrm{MHz}$, DMSO, ppm) $\delta 65.9(\mathrm{CHCHCH}), 98.1(\mathrm{CHCHCH}), 112.9,125.2,127.6,128.7$, 
129.5, 133.0 (Ar), $224.3(\mathrm{CO}) .{ }^{13} \mathrm{C}\left\{{ }^{1} \mathrm{H}\right\}$ NMR (50 MHz, $\left.\mathrm{CDCl}_{3}, \mathrm{ppm}\right) \delta 64.4$ $(\mathrm{CHCHCH}), 96.6(\mathrm{CHCHCH}), 113.0,125.5,127.4,128.9,129.3,133.7(\mathrm{Ar})$, 224.0 (CO). ${ }^{13} \mathrm{C}\left\{{ }^{1} \mathrm{H}\right\}$ NMR (50 MHz, $\left.\mathrm{CD}_{2} \mathrm{Cl}_{2}, \mathrm{ppm}\right) \delta 65.2(\mathrm{CHCHCH}), 97.5$ ( $\mathrm{CHCHCH}), 113.7,126.1,128.0,129.9,130.0,134.3(\mathrm{Ar}), 224.7$ (CO). IR (KBr, $\mathbf{c m}^{-1}$ ) 2008, 1926 (CO), 1603 (C=C). IR (Nujol, $\mathbf{~ c m}^{-1}$ ) 2008, 1916 (CO). IR (thin film, cm ${ }^{-1}$ ) 2008, 1926 (CO), 1603 (C=C). MS m/z $412\left(M^{+}\right), 273\left(M^{+}-\right.$ $\left.\mathrm{Mn}(\mathrm{CO})_{3}\right)$. Electronic spectrum $\left(\mathrm{CH}_{2} \mathrm{Cl}_{2}\right) \lambda_{\max } 511 \mathrm{~nm}(\log \varepsilon$ 3.86) Anal. Calcd for $\mathrm{C}_{22} \mathrm{H}_{13} \mathrm{O}_{3} \mathrm{~S}_{2} \mathrm{Mn}: \mathrm{C}, 64.10 ; \mathrm{H}, 3.16$. Found: $\mathrm{C}, 63.41, \mathrm{H}, 3.06$.

Synthesis of $\left(\eta^{5}-1,3-p\right.$-tolylcyclopenta[c]thienyl)tricarbonyl manganese (3d). To a $125-\mathrm{mL}$ Schlenk flask, $\mathbf{5 d}(112.5 \mathrm{mg}, 0.256 \mathrm{mmol})$ was added to a stirred suspension of $\mathrm{P}_{4} \mathrm{~S}_{10}(569 \mathrm{mg}, 1.28 \mathrm{mmol})$ and $\mathrm{NaHCO}_{3}(117 \mathrm{mg}, 1.39 \mathrm{mmol})$ in $40 \mathrm{~mL}$ of $\mathrm{CS}_{2}$. The reaction mixture was allowed to reflux for 2 hours, cooled to room temperature, and filtered through a thick pad of alumina. The volatiles were removed in vacuo. The crude product was triturated with cold pentane to give $\mathbf{3 d}$ (37.5 mg, 33.3\%) as a red solid. Mp: $118-120{ }^{\circ} \mathrm{C} .{ }^{1} \mathrm{H}$ NMR $\left(200 \mathrm{MHz}, \mathrm{C}_{6} \mathrm{D}_{6}, \mathrm{ppm}\right) \delta 2.11(\mathrm{~s}, 6 \mathrm{H}, \mathrm{Me})$, $4.75(\mathrm{t}, 1 \mathrm{H}, \mathrm{J}=3.0 \mathrm{~Hz}, \mathrm{CHCHCH}), 4.84(\mathrm{~d}, 2 \mathrm{H}, \mathrm{J}=3.0 \mathrm{~Hz}, \mathrm{CHCHCH}), 7.03(\mathrm{~d}, 4 \mathrm{H}, \mathrm{J}=$ $8.0 \mathrm{~Hz}, \mathrm{Ar}), 7.45(\mathrm{~d}, 4 \mathrm{H}, \mathrm{J}=8.0 \mathrm{~Hz}, \mathrm{Ar}) .{ }^{13} \mathrm{C}\left\{{ }^{1} \mathrm{H}\right\}$ NMR $\left(50 \mathrm{MHz}, \mathrm{C}_{6} \mathrm{D}_{6}, \mathrm{ppm}\right) \delta 21.5$ (Me), $87.9(\mathrm{CHCHCH}), 96.9(\mathrm{CHCHCH}), 113.5(\mathrm{CC}), 126.1,130.5,131.9,137.6(\mathrm{Ar})$, 224.8 (CO). IR (KBr, cm $\left.{ }^{-1}\right)$ 2007, 1929, 1896 (CO). MS (EI) m/z $440\left(\mathrm{M}^{+}\right)$. Anal. Calcd for $\mathrm{C}_{24} \mathrm{H}_{17} \mathrm{SO}_{3} \mathrm{Mn}$ : $\mathrm{C}, 65.46 ; \mathrm{H}, 3.89$. Found: $\mathrm{C}, 65.35 ; \mathrm{H}, 4.30$. Electronic spectrum $\left(\mathrm{CH}_{2} \mathrm{Cl}_{2}\right) \lambda_{\max }(\log \varepsilon) 518 \mathrm{~nm}(3.79)$.

Synthesis of $\left(\eta^{5}-1,3-a n i s y l c y c l o p e n t a[c]\right.$ thienyl)tricarbonyl manganese (3e). To a 125-mL Schlenk flask, 5e $(122 \mathrm{mg}, 0.258 \mathrm{mmol})$ was added to a stirred 
suspension of $\mathrm{P}_{4} \mathrm{~S}_{10}(1.15 \mathrm{~g}, 2.58 \mathrm{mmol})$ and $\mathrm{NaHCO}_{3}(239 \mathrm{mg}, 2.84 \mathrm{mmol})$ in $40 \mathrm{~mL}$ of $\mathrm{CS}_{2}$. The reaction mixture was allowed to reflux for 2 hours and cooled to room temperature. The reaction mixture was then passed through a thick pad of alumina and the volatiles were removed in vacuo. The crude product was triturated with cold pentane to give $3 \mathrm{e}(25.7 \mathrm{mg}, 21.4 \%)$ as a purple solid. $\mathrm{Mp}: 80-102{ }^{\circ} \mathrm{C}$ dec. ${ }^{1} \mathbf{H}$ NMR $(200 \mathrm{MHz}$, $\left.\mathrm{C}_{6} \mathrm{D}_{6}, \mathrm{ppm}\right) \delta 3.30(\mathrm{~s}, 6 \mathrm{H}, \mathrm{MeO}), 4.79(\mathrm{t}, 1 \mathrm{H}, \mathrm{J}=2.8 \mathrm{~Hz}, \mathrm{CHCHCH}), 4.87(\mathrm{~d}, 2 \mathrm{H}, \mathrm{J}=2.8$ $\mathrm{Hz}, \mathrm{CHCHCH}), 6.84\left(\mathrm{dt}, 4 \mathrm{H},{ }^{1} \mathrm{~J}=7 \mathrm{~Hz},{ }^{2} \mathrm{~J}=2.2 \mathrm{~Hz}, m-\mathrm{Ar}\right), 7.64\left(\mathrm{dt}, 4 \mathrm{H},{ }^{1} \mathrm{~J}=7 \mathrm{~Hz},{ }^{2} \mathrm{~J}=\right.$ $1.8 \mathrm{~Hz}$, o-Ar). ${ }^{13} \mathrm{C}\left\{{ }^{1} \mathrm{H}\right\}$ NMR (50 MHz, $\left.\mathrm{C}_{6} \mathrm{D}_{6}, \mathrm{ppm}\right) \delta 55.3(\mathrm{MeO}), 64.7(\mathrm{CHCHCH}), 96.8$ (CHCHCH), 114.3 (CC), 115.0 (ArCS), 115.5, 127.5, 131.7159 .8 (Ar), 224.9 (CO). IR $\left(\mathrm{KBr}, \mathbf{c m}^{-1}\right)$ 2018, $1932(\mathrm{CO})$. MS (EI) m/z $472\left(\mathrm{M}^{+}\right)$. Anal. Calcd for $\mathrm{C}_{24} \mathrm{H}_{17} \mathrm{SO}_{5} \mathrm{Mn}: \mathrm{C}$, 61.03; $\mathrm{H}, 3.63$. Found: $\mathrm{C}, 61.32 ; \mathrm{H}, 4.64$. Electronic spectrum $\left(\mathrm{CH}_{2} \mathrm{Cl}_{2}\right) \lambda_{\max }(\log \varepsilon)$ $530 \mathrm{~nm}(3.83)$.

Synthesis of $\left[\mathrm{Mn}\left\{\eta^{5}-\mathrm{SC}_{7} \mathrm{H}_{3}-1,3-\left(\mathrm{COC}_{8} \mathrm{H}_{5} \mathrm{~S}\right)_{2}\right\}(\mathrm{CO})_{3}\right]$ (3h). In a $125-\mathrm{mL}$ Schlenk flask, $5 \mathrm{~h}$ (127 mg, $0.242 \mathrm{mmol}$ ) was added to a stirred suspension of $\mathrm{P}_{4} \mathrm{~S}_{10}(539 \mathrm{mg}$, $1.21 \mathrm{mmol})$ and $\mathrm{NaHCO}_{3}(104 \mathrm{mg}, 1.24 \mathrm{mmol})$ in $40 \mathrm{~mL}$ of $\mathrm{CS}_{2}$. The reaction mixture was allowed to reflux for 2 hours and cooled to room temperature. The reaction mixture was then passed through a thick pad of alumina and the volatiles were removed in vacuo. The crude product was triturated with cold pentane to give $\mathbf{3 h}(41.8 \mathrm{mg}, 33.0 \%)$ as a purple solid. Mp: $90-91{ }^{\circ} \mathrm{C} .{ }^{1} \mathbf{H}$ NMR $\left(200 \mathrm{MHz}, \mathbf{C}_{6} \mathbf{D}_{6}, \mathbf{p p m}\right) \delta 4.73(\mathrm{~d}, 2 \mathrm{H}, \mathrm{J}=3.0$ $\mathrm{Hz}, \mathrm{CHCHCH}), 4.93(\mathrm{t}, 1 \mathrm{H}, \mathrm{J}=3.0 \mathrm{~Hz}, \mathrm{CHCHCH}), 6.92-7.03(\mathrm{~m}, 4 \mathrm{H}, \mathrm{Ar}), 7.26-7.49(\mathrm{~m}$, 4H, Ar) $7.64(\mathrm{~s}, 2 \mathrm{H}, \mathrm{Tp}) .{ }^{13} \mathrm{C}\left\{{ }^{1} \mathrm{H}\right\}$ NMR (50 MHz, $\left.\mathrm{C}_{6} \mathrm{D}_{6}, \mathrm{ppm}\right) \delta 66.3(\mathrm{CHCHCH}), 79.3$ (CHCHCH), 87.9 (CC), 120.6 (ArCS), 122.8, 123.4, 124.2, 125.5, 125.7, 126.4, 131.0, 141.0 (Ar), 224.4 (CO). IR (KBr, cm${ }^{-1}$ ) 2005, 1940, 1921 (CO). MS (EI) m/z $524\left(\mathrm{M}^{+}\right)$. 
Electronic spectrum $\left(\mathrm{CH}_{2} \mathrm{Cl}_{2}\right) \quad \lambda_{\max }(\log \varepsilon) 561 \quad \mathrm{~nm}$ (3.99). Anal. Calcd for $\mathrm{C}_{26} \mathrm{H}_{13} \mathrm{~S}_{3} \mathrm{O}_{3} \mathrm{Mn}: \mathrm{C}, 59.55 ; \mathrm{H}, 2.50$. Found: C, 59.53; H, 2.68 .

\section{References}

(1) Linn, W. J.; Sharkey, W. H. J. Am. Chem. Soc. 1957, 79, 49704972.

(2) Lloyd, D.; Preston, N. W. J. Chem. Soc. C 1969, 2464-2469.

(3) Mironov, V. A.; Fedorovich, A. D.; Yankovskii, S. A.; Luk'yanov, V. T.; Kisin, A. V.; Akhrem, A. A. Dokl. Akad. Nauk SSSR 1976, 226, 592-595 [Chem.].

(4) Wallace, C. E. The synthesis, characterization, and reactivity of some ruthenocene-fused heterocycles, Ph.D. dissertation, Department of Chemistry, University of Kentucky, 1998, 196 pp.

(5) Wallace, C. E.; Selegue, J. P.; Carrillo, A. Organometallics 1998, $17,3390-3393$.

(6) Cantrell, T. S.; Harrison, B. L. Tetrahedron Lett. 1967, 4477-4480.

(7) Quick, M. H.; Angelici, R. J.; Reimer, K. J.; Shaver, A. In Inorg. Synth.; Angelici, R. J., Ed.; Wiley: New York, 1990; Vol. 28, p 156.

(8) Pedersen, B. S.; Scheibye, S.; Nilsson, N. H.; Lawesson, S. O. Bull. Soc. Chim. Belg. 1978, 87, 223-228.

(9) Petasis, N. A.; Teets, K. A. J. Am. Chem. Soc. 1992, 114, 1032810334. 
Table S1. Crystal data and structure refinement for $\left[\mathrm{Mn}\left\{\eta^{5}-\mathrm{SC}_{7} \mathrm{H}_{3}-1,3-\mathrm{Me}_{2}\right\}(\mathrm{CO})_{3}\right]$ (3a)

\begin{tabular}{|c|c|}
\hline Identification code & k03154 \\
\hline Empirical formula & $\mathrm{C}_{12} \mathrm{H}_{9} \mathrm{Mn} \mathrm{O}_{3} \mathrm{~S}$ \\
\hline Formula weight & 288.19 \\
\hline Temperature & $90.0(2) \mathrm{K}$ \\
\hline Wavelength & $0.71073 \AA$ \\
\hline Crystal system, space group & Monoclinic, $\mathrm{P} 2_{1} / \mathrm{m}$ \\
\hline \multirow[t]{6}{*}{ Unit cell dimensions } & $a=6.4450(3) \AA$ \\
\hline & $\alpha=90.000(2)^{\circ}$ \\
\hline & $\mathrm{b}=9.0870(4) \AA$ \\
\hline & $\beta=96.7940(19)^{\circ}$ \\
\hline & $c=9.9650(6) \AA$ \\
\hline & $\gamma=90.000(2)^{\circ}$ \\
\hline Volume & $579.51(5) \AA^{3}$ \\
\hline Z, Calculated density & $2,1.652 \mathrm{Mg} / \mathrm{m}^{3}$ \\
\hline Absorption coefficient & $1.309 \mathrm{~mm}^{-1}$ \\
\hline $\mathrm{F}(000)$ & 292 \\
\hline Crystal size & $0.20 \times 0.10 \times 0.10 \mathrm{~mm}$ \\
\hline$\theta$ range for data collection & 2.06 to $27.46^{\circ}$ \\
\hline Limiting indices & $-7 \leq h \leq 8,-11 \leq k \leq 11,-12 \leq 1 \leq 12$ \\
\hline Reflections collected / unique & $4030 / 1414[R($ int $)=0.0323]$ \\
\hline Completeness to $\theta=27.46$ & $99.9 \%$ \\
\hline Absorption correction & Semi-empirical from equivalents \\
\hline Max. and min. transmission & 0.8802 and 0.7797 \\
\hline Refinement method & Full-matrix least-squares on $\mathrm{F}^{2}$ \\
\hline Data / restraints / parameters & 1414 / 0 / 86 \\
\hline Goodness-of-fit on $\mathrm{F}^{2}$ & 1.038 \\
\hline Final $R$ indices $[I>2 \sigma(I)]$ & $\mathrm{R} 1=0.0320, w R 2=0.0716$ \\
\hline $\mathrm{R}$ indices (all data) & $R 1=0.0459, w R 2=0.0767$ \\
\hline Largest diff. peak and hole & 0.601 and -0.325 e. $\AA^{-3}$ \\
\hline
\end{tabular}


Table S2. Atomic coordinates $\left(\times 10^{4}\right)$ and equivalent isotropic displacement parameters $\left(\AA^{2} \times 10^{3}\right)$ for $\left[\mathrm{Mn}\left\{\eta^{5}-\mathrm{SC}_{7} \mathrm{H}_{3}-1,3-\mathrm{Me}_{2}\right\}(\mathrm{CO})_{3}\right](3 \mathrm{a})$

\begin{tabular}{|l|l|l|l|l|}
\hline & $x$ & $y$ & $z$ & $U(e q)$ \\
\hline Mn & $356(1)$ & 2500 & $8771(1)$ & $17(1)$ \\
\hline S & $2591(1)$ & 2500 & $5179(1)$ & $21(1)$ \\
\hline O1 & $-2358(2)$ & $27(2)$ & $7721(2)$ & $26(1)$ \\
\hline O2 & $-1537(4)$ & 2500 & $11307(2)$ & $26(1)$ \\
\hline C1 & $2772(3)$ & $1089(2)$ & $6337(2)$ & $20(1)$ \\
\hline C2 & $2962(3)$ & $1703(2)$ & $7633(2)$ & $17(1)$ \\
\hline C3 & $3141(3)$ & $1221(2)$ & $9028(2)$ & $19(1)$ \\
\hline C4 & $3380(5)$ & 2500 & $9846(3)$ & $20(1)$ \\
\hline C5 & $2663(4)$ & $-485(2)$ & $5949(2)$ & $24(1)$ \\
\hline C6 & $-1327(3)$ & $1010(3)$ & $8120(2)$ & $20(1)$ \\
\hline C7 & $-783(5)$ & 2500 & $10312(3)$ & $20(1)$ \\
\hline
\end{tabular}

$\mathrm{U}(\mathrm{eq})$ is defined as one third of the trace of the orthogonalized $\mathrm{U}_{\mathrm{ij}}$ tensor 
Table S3. Bond lengths $[\AA]$ and angles $\left[{ }^{\circ}\right]$ for $\left[\mathrm{Mn}\left\{\eta^{5}-\mathrm{SC}_{7} \mathrm{H}_{3}-1,3-\mathrm{Me}_{2}\right\}(\mathrm{CO})_{3}\right](\mathbf{3 a})$

\begin{tabular}{|l|l|}
\hline $\mathrm{Mn}-\mathrm{C} 7$ & $1.778(3)$ \\
\hline $\mathrm{Mn}-\mathrm{C} 6$ & $1.807(2)$ \\
\hline $\mathrm{Mn}-\mathrm{C} 4$ & $2.110(3)$ \\
\hline $\mathrm{Mn}-\mathrm{C} 3$ & $2.129(2)$ \\
\hline $\mathrm{Mn}-\mathrm{C} 2$ & $2.2546(19)$ \\
\hline $\mathrm{S}-\mathrm{C} 1$ & $1.719(2)$ \\
\hline $\mathrm{O} 2-\mathrm{C} 7$ & $1.155(4)$ \\
\hline $\mathrm{O} 1-\mathrm{C} 6$ & $1.155(3)$ \\
\hline $\mathrm{C} 1-\mathrm{C} 2$ & $1.399(3)$ \\
\hline $\mathrm{C} 1-\mathrm{C} 5$ & $1.481(3)$ \\
\hline $\mathrm{C} 2-\mathrm{C} 3$ & $1.449(3)$ \\
\hline $\mathrm{C} 2-\mathrm{C} 2 \mathrm{~A}$ & $1.449(4)$ \\
\hline $\mathrm{C} 3-\mathrm{C} 4$ & $1.417(3)$ \\
\hline $\mathrm{C} 7-\mathrm{Mn}-\mathrm{C} 6$ & $91.06(9)$ \\
\hline $\mathrm{C} 6-\mathrm{Mn}-\mathrm{C} 6 \mathrm{~A}$ & $97.10(14)$ \\
\hline $\mathrm{C} 7-\mathrm{Mn}-\mathrm{C} 4$ & $90.74(13)$ \\
\hline $\mathrm{C} 6-\mathrm{Mn}-\mathrm{C} 4$ & $131.41(7)$ \\
\hline $\mathrm{C} 6-\mathrm{Mn}-\mathrm{C} 3 \mathrm{~A}$ & $156.12(8)$ \\
\hline $\mathrm{C} 6 \mathrm{~A}-\mathrm{Mn}-\mathrm{C} 3 \mathrm{~A}$ & $95.49(9)$ \\
\hline $\mathrm{C} 4-\mathrm{Mn}-\mathrm{C} 3 \mathrm{~A}$ & $39.07(7)$ \\
\hline $\mathrm{C} 7-\mathrm{Mn}-\mathrm{C} 3$ & $108.94(10)$ \\
\hline $\mathrm{C} 6-\mathrm{Mn}-\mathrm{C} 3$ & $95.50(9)$ \\
\hline $\mathrm{C} 6 \mathrm{~A}-\mathrm{Mn}-\mathrm{C} 3$ & $156.12(9)$ \\
\hline $\mathrm{C} 4-\mathrm{Mn}-\mathrm{C} 3$ & $39.07(7)$ \\
\hline $\mathrm{C} 3 \mathrm{~A}-\mathrm{Mn}-\mathrm{C} 3$ & $66.20(12)$ \\
\hline $\mathrm{C} 7-\mathrm{Mn}-\mathrm{C} 2$ & $147.39(9)$ \\
\hline $\mathrm{C} 6-\mathrm{Mn}-\mathrm{C} 2$ & $91.71(8)$ \\
\hline $\mathrm{C} 6 \mathrm{~A}-\mathrm{Mn}-\mathrm{C} 2$ & $120.78(8)$ \\
\hline & \\
\hline
\end{tabular}

\begin{tabular}{|l|l|}
\hline $\mathrm{C} 4-\mathrm{Mn}-\mathrm{C} 2$ & $63.68(9)$ \\
\hline $\mathrm{C} 3 \mathrm{~A}-\mathrm{Mn}-\mathrm{C} 2$ & $64.41(8)$ \\
\hline $\mathrm{C} 3-\mathrm{Mn}-\mathrm{C} 2$ & $38.46(7)$ \\
\hline $\mathrm{C} 7-\mathrm{Mn}-\mathrm{C} 2 \mathrm{~A}$ & $147.39(10)$ \\
\hline $\mathrm{C} 6-\mathrm{Mn}-\mathrm{C} 2 \mathrm{~A}$ & $120.77(8)$ \\
\hline $\mathrm{C} 6 \mathrm{~A}-\mathrm{Mn}-\mathrm{C} 2 \mathrm{~A}$ & $91.71(8)$ \\
\hline $\mathrm{C} 4-\mathrm{Mn}-\mathrm{C} 2 \mathrm{~A}$ & $63.68(9)$ \\
\hline $\mathrm{C} 3 \mathrm{~A}-\mathrm{Mn}-\mathrm{C} 2 \mathrm{~A}$ & $38.46(7)$ \\
\hline $\mathrm{C} 3-\mathrm{Mn}-\mathrm{C} 2 \mathrm{~A}$ & $64.41(8)$ \\
\hline $\mathrm{C} 2-\mathrm{Mn}-\mathrm{C} 2 \mathrm{~A}$ & $37.50(10)$ \\
\hline $\mathrm{C} 1-\mathrm{S}-\mathrm{C} 1 \mathrm{~A}$ & $96.44(14)$ \\
\hline $\mathrm{C} 3-\mathrm{C} 4-\mathrm{C} 3 \mathrm{~A}$ & $110.2(2)$ \\
\hline $\mathrm{C} 3-\mathrm{C} 4-\mathrm{Mn}$ & $71.17(14)$ \\
\hline $\mathrm{C} 3 \mathrm{~A}-\mathrm{C} 4-\mathrm{Mn}$ & $71.17(14)$ \\
\hline $\mathrm{C} 4-\mathrm{C} 3-\mathrm{C} 2$ & $107.13(19)$ \\
\hline $\mathrm{C} 4-\mathrm{C} 3-\mathrm{Mn}$ & $69.76(14)$ \\
\hline $\mathrm{C} 2-\mathrm{C} 3-\mathrm{Mn}$ & $75.47(11)$ \\
\hline $\mathrm{C} 1-\mathrm{C} 2-\mathrm{C} 3$ & $138.9(2)$ \\
\hline $\mathrm{C} 1-\mathrm{C} 2-\mathrm{C} 2 \mathrm{~A}$ & $113.47(12)$ \\
\hline $\mathrm{C} 3-\mathrm{C} 2-\mathrm{C} 2 \mathrm{~A}$ & $107.59(12)$ \\
\hline $\mathrm{C} 1-\mathrm{C} 2-\mathrm{Mn}$ & $127.14(14)$ \\
\hline $\mathrm{C} 3-\mathrm{C} 2-\mathrm{Mn}$ & $66.07(11)$ \\
\hline $\mathrm{C} 2 \mathrm{~A}-\mathrm{C} 2-\mathrm{Mn}$ & $71.25(5)$ \\
\hline $\mathrm{C} 2-\mathrm{C} 1-\mathrm{C} 5$ & $128.43(19)$ \\
\hline $\mathrm{C} 2-\mathrm{C} 1-\mathrm{S}$ & $108.31(15)$ \\
\hline $\mathrm{C} 5-\mathrm{C} 1-\mathrm{S}$ & $123.23(16)$ \\
\hline $\mathrm{O} 2-\mathrm{C} 7-\mathrm{Mn}$ & $179.5(3)$ \\
\hline $\mathrm{O} 1-\mathrm{C} 6-\mathrm{Mn}$ & $177.92(19)$ \\
\hline & \\
\hline
\end{tabular}


Table S4. Anisotropic displacement parameters $\left(\AA^{2} \times 10^{3}\right)$ for $\left[\mathrm{Mn}\left\{\eta^{5}-\mathrm{SC}_{7} \mathrm{H}_{3}-1,3-\right.\right.$ $\left.\left.\mathrm{Me}_{2}\right\}(\mathrm{CO})_{3}\right](3 \mathbf{a})$

\begin{tabular}{|l|l|l|l|l|l|l|}
\hline Atom & $\mathrm{U}_{11}$ & $\mathrm{U}_{22}$ & $\mathrm{U}_{33}$ & $\mathrm{U}_{23}$ & $\mathrm{U}_{13}$ & $\mathrm{U}_{12}$ \\
\hline $\mathrm{Mn}$ & $18(1)$ & $17(1)$ & $16(1)$ & 0 & $2(1)$ & 0 \\
\hline $\mathrm{S}$ & $25(1)$ & $18(1)$ & $20(1)$ & 0 & $8(1)$ & 0 \\
\hline $\mathrm{O} 2$ & $35(1)$ & $24(1)$ & $21(1)$ & 0 & $10(1)$ & 0 \\
\hline $\mathrm{O} 1$ & $25(1)$ & $25(1)$ & $27(1)$ & $-5(1)$ & $2(1)$ & $-6(1)$ \\
\hline C4 & $16(2)$ & $23(2)$ & $18(1)$ & 0 & $-3(1)$ & 0 \\
\hline $\mathrm{C} 3$ & $18(1)$ & $19(1)$ & $20(1)$ & $3(1)$ & $1(1)$ & $2(1)$ \\
\hline C2 & $13(1)$ & $18(1)$ & $20(1)$ & $0(1)$ & $2(1)$ & $2(1)$ \\
\hline C1 & $19(1)$ & $17(1)$ & $23(1)$ & $1(1)$ & $4(1)$ & $1(1)$ \\
\hline C5 & $31(1)$ & $20(1)$ & $22(1)$ & $-1(1)$ & $5(1)$ & $-1(1)$ \\
\hline C7 & $22(2)$ & $14(2)$ & $22(2)$ & 0 & $-4(1)$ & 0 \\
\hline C6 & $21(1)$ & $25(1)$ & $16(1)$ & $1(1)$ & $6(1)$ & $6(1)$ \\
\hline
\end{tabular}

The anisotropic displacement factor exponent takes the form: $-2 \pi^{2}\left[h^{2} a^{* 2} U_{11}+\right.$ $\left.\ldots+2 \mathrm{~h} \mathrm{k} \mathrm{a}^{*} \mathrm{~b}^{*} \mathrm{U}_{12}\right]$ 
Table S5. Hydrogen coordinates $\left(\times 10^{4}\right)$ and isotropic displacement parameters $\left(\mathrm{A}^{2} \times 10^{3}\right)$ for $\left[\mathrm{Mn}\left\{\eta^{5}-\mathrm{SC}_{7} \mathrm{H}_{3}-1,3-\mathrm{Me}_{2}\right\}(\mathrm{CO})_{3}\right]$ (3a)

\begin{tabular}{|l|l|l|l|l|}
\hline & $x$ & $y$ & $z$ & $U(e q)$ \\
\hline H1 & 3661 & 2500 & 10803 & 24 \\
\hline H2 & 3105 & 233 & 9337 & 23 \\
\hline H5A & 3872 & -1007 & 6413 & 36 \\
\hline H5B & 2668 & -574 & 4970 & 36 \\
\hline H5C & 1375 & -917 & 6209 & 36 \\
\hline
\end{tabular}


Table S6. Torsion angles [deg] for $\left[\mathrm{Mn}\left\{\eta^{5}-\mathrm{SC}_{7} \mathrm{H}_{3}-1,3-\mathrm{Me}_{2}\right\}(\mathrm{CO})_{3}\right]$ (3a)

\begin{tabular}{|l|l|}
\hline $\mathrm{C} 7-\mathrm{Mn}-\mathrm{C} 4-\mathrm{C} 3$ & $119.95(12)$ \\
\hline $\mathrm{C} 6-\mathrm{Mn}-\mathrm{C} 4-\mathrm{C} 3$ & $27.9(2)$ \\
\hline $\mathrm{C} 6 \mathrm{~A}-\mathrm{Mn}-\mathrm{C} 4-\mathrm{C} 3$ & $-147.99(12)$ \\
\hline $\mathrm{C} 3 \mathrm{~A}-\mathrm{Mn}-\mathrm{C} 4-\mathrm{C} 3$ & $-120.1(2)$ \\
\hline $\mathrm{C} 2-\mathrm{Mn}-\mathrm{C} 4-\mathrm{C} 3$ & $-39.04(12)$ \\
\hline C2A-Mn-C4-C3 & $-81.07(14)$ \\
\hline $\mathrm{C} 7-\mathrm{Mn}-\mathrm{C} 4-\mathrm{C} 3 \mathrm{~A}$ & $-119.95(12)$ \\
\hline $\mathrm{C} 6-\mathrm{Mn}-\mathrm{C} 4-\mathrm{C} 3 \mathrm{~A}$ & $147.99(12)$ \\
\hline $\mathrm{C} 6 \mathrm{~A}-\mathrm{Mn}-\mathrm{C} 4-\mathrm{C} 3 \mathrm{~A}$ & $-27.9(2)$ \\
\hline $\mathrm{C} 3-\mathrm{Mn}-\mathrm{C} 4-\mathrm{C} 3 \mathrm{~A}$ & $120.1(2)$ \\
\hline $\mathrm{C} 2-\mathrm{Mn}-\mathrm{C} 4-\mathrm{C} 3 \mathrm{~A}$ & $81.07(14)$ \\
\hline $\mathrm{C} 2 \mathrm{~A}-\mathrm{Mn}-\mathrm{C} 4-\mathrm{C} 3 \mathrm{~A}$ & $39.04(12)$ \\
\hline $\mathrm{C} 3 \mathrm{~A}-\mathrm{C} 4-\mathrm{C} 3-\mathrm{C} 2$ & $6.1(3)$ \\
\hline $\mathrm{Mn}-\mathrm{C} 4-\mathrm{C} 3-\mathrm{C} 2$ & $66.83(14)$ \\
\hline $\mathrm{C} 3 \mathrm{~A}-\mathrm{C} 4-\mathrm{C} 3-\mathrm{Mn}$ & $-60.7(2)$ \\
\hline $\mathrm{C} 7-\mathrm{Mn}-\mathrm{C} 3-\mathrm{C} 4$ & $-66.35(15)$ \\
\hline $\mathrm{C} 6-\mathrm{Mn}-\mathrm{C} 3-\mathrm{C} 4$ & $-159.36(14)$ \\
\hline $\mathrm{C} 6 \mathrm{~A}-\mathrm{Mn}-\mathrm{C} 3-\mathrm{C} 4$ & $79.0(2)$ \\
\hline $\mathrm{C} 3 \mathrm{~A}-\mathrm{Mn}-\mathrm{C} 3-\mathrm{C} 4$ & $36.58(14)$ \\
\hline $\mathrm{C} 2-\mathrm{Mn}-\mathrm{C} 3-\mathrm{C} 4$ & $114.82(18)$ \\
\hline $\mathrm{C} 2 \mathrm{~A}-\mathrm{Mn}-\mathrm{C} 3-\mathrm{C} 4$ & $79.05(14)$ \\
\hline $\mathrm{C} 7-\mathrm{Mn}-\mathrm{C} 3-\mathrm{C} 2$ & $178.83(11)$ \\
\hline $\mathrm{C} 6-\mathrm{Mn}-\mathrm{C} 3-\mathrm{C} 2$ & $85.82(13)$ \\
\hline $\mathrm{C} 6 \mathrm{~A}-\mathrm{Mn}-\mathrm{C} 3-\mathrm{C} 2$ & $-35.8(3)$ \\
\hline $\mathrm{C} 4-\mathrm{Mn}-\mathrm{C} 3-\mathrm{C} 2$ & $-114.82(18)$ \\
\hline $\mathrm{C} 3 \mathrm{~A}-\mathrm{Mn}-\mathrm{C} 3-\mathrm{C} 2$ & $-78.25(12)$ \\
\hline $\mathrm{C} 2 \mathrm{~A}-\mathrm{Mn}-\mathrm{C} 3-\mathrm{C} 2$ & $-35.78(12)$ \\
\hline $\mathrm{C} 4-\mathrm{C} 3-\mathrm{C} 2-\mathrm{C} 1$ & $177.2(2)$ \\
\hline $\mathrm{Mn}-\mathrm{C} 3-\mathrm{C} 2-\mathrm{C} 1$ & $-119.7(3)$ \\
\hline $\mathrm{C} 4-\mathrm{C} 3-\mathrm{C} 2-\mathrm{C} 2 \mathrm{~A}$ & $-3.65(19)$ \\
\hline $\mathrm{Mn}-\mathrm{C} 3-\mathrm{C} 2-\mathrm{C} 2 \mathrm{~A}$ & $59.36(6)$ \\
\hline $\mathrm{C} 4-\mathrm{C} 3-\mathrm{C} 2-\mathrm{Mn}$ & $-63.01(16)$ \\
\hline $\mathrm{C} 7-\mathrm{Mn}-\mathrm{C} 2-\mathrm{C} 1$ & $132.26(19)$ \\
\hline $\mathrm{C} 6-\mathrm{Mn}-\mathrm{C} 2-\mathrm{C} 1$ & $37.63(19)$ \\
\hline $\mathrm{C} 6 \mathrm{~A}-\mathrm{Mn}-\mathrm{C} 2-\mathrm{C} 1$ & $-61.7(2)$ \\
\hline $\mathrm{C} 4-\mathrm{Mn}-\mathrm{C} 2-\mathrm{C} 1$ & $174.0(2)$ \\
\hline $\mathrm{C} 3 \mathrm{~A}-\mathrm{Mn}-\mathrm{C} 2-\mathrm{C} 1$ & $-142.4(2)$ \\
\hline & \\
\hline
\end{tabular}

\begin{tabular}{|l|l|}
\hline $\mathrm{C} 3-\mathrm{Mn}-\mathrm{C} 2-\mathrm{C} 1$ & $134.3(2)$ \\
\hline $\mathrm{C} 2 \mathrm{~A}-\mathrm{Mn}-\mathrm{C} 2-\mathrm{C} 1$ & $-105.69(18)$ \\
\hline $\mathrm{C} 7-\mathrm{Mn}-\mathrm{C} 2-\mathrm{C} 3$ & $-2.1(2)$ \\
\hline $\mathrm{C} 6-\mathrm{Mn}-\mathrm{C} 2-\mathrm{C} 3$ & $-96.69(13)$ \\
\hline $\mathrm{C} 6 \mathrm{~A}-\mathrm{Mn}-\mathrm{C} 2-\mathrm{C} 3$ & $164.00(13)$ \\
\hline $\mathrm{C} 4-\mathrm{Mn}-\mathrm{C} 2-\mathrm{C} 3$ & $39.65(11)$ \\
\hline $\mathrm{C} 3 \mathrm{~A}-\mathrm{Mn}-\mathrm{C} 2-\mathrm{C} 3$ & $83.31(16)$ \\
\hline $\mathrm{C} 2 \mathrm{~A}-\mathrm{Mn}-\mathrm{C} 2-\mathrm{C} 3$ & $119.99(11)$ \\
\hline $\mathrm{C} 7-\mathrm{Mn}-\mathrm{C} 2-\mathrm{C} 2 \mathrm{~A}$ & $-122.04(14)$ \\
\hline $\mathrm{C} 6-\mathrm{Mn}-\mathrm{C} 2-\mathrm{C} 2 \mathrm{~A}$ & $143.32(7)$ \\
\hline $\mathrm{C} 6 \mathrm{~A}-\mathrm{Mn}-\mathrm{C} 2-\mathrm{C} 2 \mathrm{~A}$ & $44.02(8)$ \\
\hline $\mathrm{C} 4-\mathrm{Mn}-\mathrm{C} 2-\mathrm{C} 2 \mathrm{~A}$ & $-80.34(5)$ \\
\hline $\mathrm{C} 3 \mathrm{~A}-\mathrm{Mn}-\mathrm{C} 2-\mathrm{C} 2 \mathrm{~A}$ & $-36.68(7)$ \\
\hline $\mathrm{C} 3-\mathrm{Mn}-\mathrm{C} 2-\mathrm{C} 2 \mathrm{~A}$ & $-119.99(11)$ \\
\hline $\mathrm{C} 3-\mathrm{C} 2-\mathrm{C} 1-\mathrm{C} 5$ & $0.8(4)$ \\
\hline $\mathrm{C} 2 \mathrm{~A}-\mathrm{C} 2-\mathrm{C} 1-\mathrm{C} 5$ & $-178.30(18)$ \\
\hline $\mathrm{Mn}-\mathrm{C} 2-\mathrm{C} 1-\mathrm{C} 5$ & $-94.7(3)$ \\
\hline $\mathrm{C} 3-\mathrm{C} 2-\mathrm{C} 1-\mathrm{S}$ & $178.8(2)$ \\
\hline $\mathrm{C} 2 \mathrm{~A}-\mathrm{C} 2-\mathrm{C} 1-\mathrm{S}$ & $-0.28(15)$ \\
\hline $\mathrm{Mn}-\mathrm{C} 2-\mathrm{C} 1-\mathrm{S}$ & $83.36(18)$ \\
\hline $\mathrm{C} 1 \mathrm{~A}-\mathrm{S}-\mathrm{C} 1-\mathrm{C} 2$ & $0.4(2)$ \\
\hline $\mathrm{C} 1 \mathrm{~A}-\mathrm{S}-\mathrm{C} 1-\mathrm{C} 5$ & $178.53(13)$ \\
\hline $\mathrm{C} 6-\mathrm{Mn}-\mathrm{C} 7-\mathrm{O} 1$ & $-48.56(17)$ \\
\hline $\mathrm{C} 6 \mathrm{~A}-\mathrm{Mn}-\mathrm{C} 7-\mathrm{O} 1$ & $48.56(17)$ \\
\hline $\mathrm{C} 4-\mathrm{Mn}-\mathrm{C} 7-\mathrm{O} 1$ & $180.00(16)$ \\
\hline $\mathrm{C} 3 \mathrm{~A}-\mathrm{Mn}-\mathrm{C} 7-\mathrm{O} 1$ & $144.74(16)$ \\
\hline $\mathrm{C} 3-\mathrm{Mn}-\mathrm{C} 7-\mathrm{O} 1$ & $-144.74(18)$ \\
\hline $\mathrm{C} 2-\mathrm{Mn}-\mathrm{C} 7-\mathrm{O} 1$ & $-143.4(2)$ \\
\hline $\mathrm{C} 2 \mathrm{~A}-\mathrm{Mn}-\mathrm{C} 7-\mathrm{O} 1$ & $143.4(2)$ \\
\hline $\mathrm{C} 7-\mathrm{Mn}-\mathrm{C} 6-\mathrm{O} 2$ & $-89(5)$ \\
\hline $\mathrm{C} 6 \mathrm{~A}-\mathrm{Mn}-\mathrm{C} 6-\mathrm{O} 2$ & $179(100)$ \\
\hline $\mathrm{C} 4-\mathrm{Mn}-\mathrm{C} 6-\mathrm{O} 2$ & $2(5)$ \\
\hline $\mathrm{C} 3 \mathrm{~A}-\mathrm{Mn}-\mathrm{C} 6-\mathrm{O} 2$ & $58(5)$ \\
\hline $\mathrm{C} 3-\mathrm{Mn}-\mathrm{C} 6-\mathrm{O} 2$ & $20(5)$ \\
\hline $\mathrm{C} 2-\mathrm{Mn}-\mathrm{C} 6-\mathrm{O} 2$ & $58(5)$ \\
\hline $\mathrm{C} 2 \mathrm{~A}-\mathrm{Mn}-\mathrm{C} 6-\mathrm{O} 2$ & $83(5)$ \\
\hline & \\
\hline
\end{tabular}


Table S7. Crystal data and structure refinement for $\left[\mathrm{Mn}\left\{\eta^{5}-\mathrm{SC}_{7} \mathrm{H}_{3}-1,3-\mathrm{Ph}_{2}\right\}(\mathrm{CO})_{3}\right](3 \mathrm{c})$

\begin{tabular}{|l|l|}
\hline Identification code & $\mathrm{k} 04077$ \\
\hline Empirical formula & $\mathrm{C}_{22} \mathrm{H}_{13} \mathrm{MnO}_{3} \mathrm{~S}$ \\
\hline Formula weight & 412.32 \\
\hline Temperature & $90.0(2) \mathrm{K}$ \\
\hline Wavelength & $0.71073 \AA$ \\
\hline Crystal system, space group & triclinic, $\mathrm{P}-1$ \\
\hline Unit cell dimensions & $\mathrm{a}=11.1210(2) \AA$ \\
\hline & $\alpha=66.5710(9)^{\circ}$ \\
\hline & $\mathrm{b}=13.2140(2) \AA$ \\
\hline & $\beta=85.0370(9)^{\circ}$ \\
\hline & $\mathrm{C}=13.3160(3) \AA$ \\
\hline & $\gamma=76.7720(9)^{\circ}$ \\
\hline Volume & $1747.83(6) \AA^{3}$ \\
\hline$Z$, Calculated density & $4,1.567 \mathrm{Mg} / \mathrm{m}^{3}$ \\
\hline Absorption coefficient & $0.895 \mathrm{~mm}{ }^{-1}$ \\
\hline Absorption correction & $\mathrm{None}$ \\
\hline F(000) & 840 \\
\hline Crystal size & $0.2 \times 0.1 \times 0.04 \mathrm{~mm}$ \\
\hline$\theta$ range for data collection & 1.67 to 25.00 \\
\hline Limiting indices & $-13 \leq \mathrm{h} \leq 13,-15 \leq \mathrm{k} \leq 15,-15 \leq \mathrm{I} \leq 15$ \\
\hline Reflections collected / unique & $12168 / 6164[\mathrm{R}(\mathrm{int})=0.0710]$ \\
\hline Completeness to $\theta=25.00$ & $100.0 \%$ \\
\hline Refinement method & $\mathrm{Fulll}-\mathrm{matrix}$ least-squares on $\mathrm{F}^{2}$ \\
\hline Data / restraints / parameters & $6164 / 0 / 487$ \\
\hline Goodness-of-fit on $\mathrm{F}^{2}$ & 1.013 \\
\hline Final R indices [l>2 $\sigma(\mathrm{I})]$ & $\mathrm{R} 1=0.0552, \mathrm{wR} 2=0.1156$ \\
\hline R indices (all data) & $\mathrm{R} 1=0.1068, \mathrm{wR} 2=0.1340$ \\
\hline Largest diff. peak and hole & $0.801 \mathrm{and}-0.441 \mathrm{e} \cdot \AA^{-3}$ \\
\hline & \\
\hline
\end{tabular}


Table S8. Atomic coordinates $\left(\times 10^{4}\right)$ and equivalent isotropic displacement parameters $\left(\mathrm{A}^{2} \times 10^{3}\right)$ for $\left[\mathrm{Mn}\left\{\eta^{5}-\mathrm{SC}_{7} \mathrm{H}_{3}-1,3-\mathrm{Ph}_{2}\right\}(\mathrm{CO})_{3}\right](3 \mathrm{c})$

\begin{tabular}{|l|l|l|l|l|}
\hline Atom & $\mathrm{x}$ & $\mathrm{y}$ & $\mathrm{z}$ & $\mathrm{U}(\mathrm{eq})$ \\
\hline Mn1A & $7692(1)$ & $6575(1)$ & $2111(1)$ & $20(1)$ \\
\hline S1A & $10140(1)$ & $4050(1)$ & $4215(1)$ & $21(1)$ \\
\hline O1A & $7050(3)$ & $7104(3)$ & $4075(3)$ & $33(1)$ \\
\hline O2A & $5721(3)$ & $8439(3)$ & $838(2)$ & $28(1)$ \\
\hline O3A & $9504(3)$ & $8034(3)$ & $1194(2)$ & $28(1)$ \\
\hline C1A & $8557(4)$ & $4214(3)$ & $4253(3)$ & $19(1)$ \\
\hline C2A & $8095(4)$ & $4721(3)$ & $3176(4)$ & $18(1)$ \\
\hline C3A & $6932(4)$ & $5105(3)$ & $2602(3)$ & $19(1)$ \\
\hline C4A & $7207(4)$ & $5481(3)$ & $1463(4)$ & $22(1)$ \\
\hline C5A & $8475(4)$ & $5469(4)$ & $1303(4)$ & $21(1)$ \\
\hline C6A & $9061(4)$ & $4956(3)$ & $2363(3)$ & $17(1)$ \\
\hline C7A & $10255(4)$ & $4646(3)$ & $2812(3)$ & $18(1)$ \\
\hline C8A & $11449(4)$ & $4777(4)$ & $2294(3)$ & $18(1)$ \\
\hline C9A & $11522(4)$ & $5454(4)$ & $1173(4)$ & $24(1)$ \\
\hline C10A & $12651(4)$ & $5606(4)$ & $687(4)$ & $27(1)$ \\
\hline C11A & $13728(4)$ & $5106(4)$ & $1281(4)$ & $24(1)$ \\
\hline C12A & $13667(4)$ & $4453(4)$ & $2397(4)$ & $27(1)$ \\
\hline C13A & $12555(4)$ & $4283(4)$ & $2891(4)$ & $21(1)$ \\
\hline C14A & $7906(4)$ & $3819(3)$ & $5286(3)$ & $19(1)$ \\
\hline C15A & $8529(4)$ & $3352(4)$ & $6299(4)$ & $22(1)$ \\
\hline C16A & $7907(4)$ & $2950(4)$ & $7283(4)$ & $26(1)$ \\
\hline C17A & $6656(4)$ & $3018(4)$ & $7275(4)$ & $23(1)$ \\
\hline C18A & $6020(4)$ & $3469(4)$ & $6297(4)$ & $27(1)$ \\
\hline C19A & $6640(4)$ & $3868(4)$ & $5314(4)$ & $24(1)$ \\
\hline C20A & $7306(4)$ & $6910(4)$ & $3304(4)$ & $24(1)$ \\
\hline C21A & $6506(5)$ & $7708(4)$ & $1343(4)$ & $22(1)$ \\
\hline C22A & $8801(4)$ & $7464(4)$ & $1580(3)$ & $20(1)$ \\
\hline Mn1B & $7608(1)$ & $-1169(1)$ & $7731(1)$ & $19(1)$ \\
\hline S1B & $9813(1)$ & $852(1)$ & $5767(1)$ & $18(1)$ \\
\hline O1B & $9682(3)$ & $-3076(3)$ & $8768(2)$ & $26(1)$ \\
\hline O2B & $5918(3)$ & $-2629(3)$ & $8992(2)$ & $24(1)$ \\
\hline O3B & $7434(3)$ & $-1600(3)$ & $5736(3)$ & $31(1)$ \\
\hline C1B & $8264(4)$ & $1007(3)$ & $5606(3)$ & $18(1)$ \\
\hline C2B & $7720(4)$ & $620(3)$ & $6660(3)$ & $19(1)$ \\
\hline C3B & $6530(4)$ & $512(4)$ & $7143(4)$ & $22(1)$ \\
\hline C4B & $6679(4)$ & $95(3)$ & $8293(4)$ & $21(1)$ \\
\hline C5B & $7940(4)$ & $-162(3)$ & $8555(3)$ & $20(1)$ \\
\hline C6B & $8614(4)$ & $195(3)$ & $7540(3)$ & $20(1)$ \\
\hline C7B & $9820(4)$ & $271(3)$ & $7173(3)$ & $18(1)$ \\
\hline C8B & $10960(4)$ & $-19(3)$ & $7793(3)$ & $17(1)$ \\
\hline & & & & \\
\hline
\end{tabular}




\begin{tabular}{|l|l|l|l|l|}
\hline C9B & $11045(4)$ & $-768(4)$ & $8892(3)$ & $20(1)$ \\
\hline C10B & $12121(4)$ & $-1054(4)$ & $9482(4)$ & $23(1)$ \\
\hline C11B & $13130(4)$ & $-608(4)$ & $8991(4)$ & $24(1)$ \\
\hline C12B & $13060(4)$ & $119(4)$ & $7911(4)$ & $26(1)$ \\
\hline C13B & $12002(4)$ & $410(4)$ & $7306(4)$ & $20(1)$ \\
\hline C14B & $7682(4)$ & $1477(4)$ & $4544(4)$ & $19(1)$ \\
\hline C15B & $8299(4)$ & $2028(4)$ & $3588(4)$ & $21(1)$ \\
\hline C16B & $7741(4)$ & $2476(3)$ & $2570(3)$ & $19(1)$ \\
\hline C17B & $6541(4)$ & $2390(4)$ & $2476(4)$ & $22(1)$ \\
\hline C18B & $5919(4)$ & $1834(4)$ & $3401(4)$ & $25(1)$ \\
\hline C19B & $6479(4)$ & $1381(4)$ & $4418(4)$ & $23(1)$ \\
\hline C20B & $8888(4)$ & $-2318(4)$ & $8338(4)$ & $22(1)$ \\
\hline C21B & $6581(4)$ & $-2053(4)$ & $8479(4)$ & $19(1)$ \\
\hline C22B & $7504(4)$ & $-1456(4)$ & $6524(4)$ & $22(1)$ \\
\hline
\end{tabular}

$\mathrm{U}(\mathrm{eq})$ is defined as one third of the trace of the orthogonalized $\mathrm{U}_{\mathrm{ij}}$ tensor. 
Table S9. Bond lengths $[\AA]$ and angles $\left[^{\circ}\right]$ for $\left[\mathrm{Mn}\left\{\eta^{5}-\mathrm{SC}_{7} \mathrm{H}_{3}-1,3-\mathrm{Ph}_{2}\right\}(\mathrm{CO})_{3}\right](3 \mathbf{c})$

\begin{tabular}{|l|l|}
\hline Mn1A-C21A & $1.772(5)$ \\
\hline Mn1A-C20A & $1.806(5)$ \\
\hline Mn1A-C22A & $1.803(5)$ \\
\hline Mn1A-C4A & $2.135(4)$ \\
\hline Mn1A-C5A & $2.135(4)$ \\
\hline Mn1A-C3A & $2.137(4)$ \\
\hline Mn1A-C6A & $2.244(4)$ \\
\hline Mn1A-C2A & $2.250(4)$ \\
\hline S1A-C7A & $1.721(4)$ \\
\hline S1A-C1A & $1.722(4)$ \\
\hline O1A-C20A & $1.151(5)$ \\
\hline O2A-C21A & $1.160(5)$ \\
\hline O3A-C22A & $1.155(5)$ \\
\hline C1A-C2A & $1.405(6)$ \\
\hline C1A-C14A & $1.455(6)$ \\
\hline C2A-C3A & $1.442(6)$ \\
\hline C2A-C6A & $1.451(6)$ \\
\hline C3A-C4A & $1.425(6)$ \\
\hline C4A-C5A & $1.405(6)$ \\
\hline C5A-C6A & $1.440(6)$ \\
\hline C6A-C7A & $1.408(6)$ \\
\hline C7A-C8A & $1.456(6)$ \\
\hline C8A-C13A & $1.400(6)$ \\
\hline C8A-C9A & $1.409(6)$ \\
\hline C9A-C10A & $1.381(6)$ \\
\hline C10A-C11A & $1.373(6)$ \\
\hline C11A-C12A & $1.396(6)$ \\
\hline C12A-C13A & $1.371(6)$ \\
\hline C14A-C19A & $1.393(6)$ \\
\hline C14A-C15A & $1.408(6)$ \\
\hline C15A-C16A & $1.391(6)$ \\
\hline C16A-C17A & $1.374(6)$ \\
\hline C17A-C18A & $1.379(6)$ \\
\hline C18A-C19A & $1.389(6)$ \\
\hline Mn1B-C21B & $1.775(5)$ \\
\hline Mn1B-C20B & $1.794(5)$ \\
\hline Mn1B-C22B & $1.810(5)$ \\
\hline Mn1B-C4B & $2.118(4)$ \\
\hline Mn1B-C3B & $2.132(4)$ \\
\hline Mn1B-C5B & $2.138(4)$ \\
\hline Mn1B-C2B & $2.249(4)$ \\
\hline Mn1B-C6B & $2.253(4)$ \\
\hline
\end{tabular}

\begin{tabular}{|l|l|}
\hline S1B-C1B & $1.710(4)$ \\
\hline S1B-C7B & $1.719(4)$ \\
\hline O1B-C20B & $1.156(5)$ \\
\hline O2B-C21B & $1.160(5)$ \\
\hline O3B-C22B & $1.152(5)$ \\
\hline C1B-C2B & $1.422(6)$ \\
\hline C1B-C14B & $1.442(6)$ \\
\hline C2B-C3B & $1.432(6)$ \\
\hline C2B-C6B & $1.449(6)$ \\
\hline C3B-C4B & $1.416(6)$ \\
\hline C4B-C5B & $1.406(6)$ \\
\hline C5B-C6B & $1.446(6)$ \\
\hline C6B-C7B & $1.400(6)$ \\
\hline C7B-C8B & $1.463(6)$ \\
\hline C8B-C9B & $1.400(6)$ \\
\hline C8B-C13B & $1.401(6)$ \\
\hline C9B-C10B & $1.383(6)$ \\
\hline C10B-C11B & $1.380(6)$ \\
\hline C11B-C12B & $1.372(6)$ \\
\hline C12B-C13B & $1.376(6)$ \\
\hline C14B-C15B & $1.400(6)$ \\
\hline C14B-C19B & $1.403(6)$ \\
\hline C15B-C16B & $1.382(6)$ \\
\hline C16B-C17B & $1.385(6)$ \\
\hline C17B-C18B & $1.379(6)$ \\
\hline C18B-C19B & $1.383(6)$ \\
\hline C21A-Mn1A-C20A & $91.0(2)$ \\
\hline C21A-Mn1A-C22A & $90.09(19)$ \\
\hline C20A-Mn1A-C22A & $95.48(19)$ \\
\hline C21A-Mn1A-C4A & $91.49(19)$ \\
\hline C20A-Mn1A-C4A & $136.58(18)$ \\
\hline C22A-Mn1A-C4A & $127.85(18)$ \\
\hline C21A-Mn1A-C5A & $113.32(18)$ \\
\hline C20A-Mn1A-C5A & $153.57(19)$ \\
\hline C22A-Mn1A-C5A & $94.77(18)$ \\
\hline C4A-Mn1A-C5A & $38.43(16)$ \\
\hline C21A-Mn1A-C3A & $105.09(18)$ \\
\hline C20A-Mn1A-C3A & $99.05(18)$ \\
\hline C22A-Mn1A-C3A & $158.70(18)$ \\
\hline C4A-Mn1A-C3A & $38.97(15)$ \\
\hline C5A-Mn1A-C3A & $65.70(16)$ \\
\hline C21A-Mn1A-C6A & $151.41(18)$ \\
\hline
\end{tabular}




\begin{tabular}{|l|l|}
\hline C20A-Mn1A-C6A & $116.35(18)$ \\
\hline C22A-Mn1A-C6A & $95.20(17)$ \\
\hline C4A-Mn1A-C6A & $63.26(16)$ \\
\hline C5A-Mn1A-C6A & $38.30(15)$ \\
\hline C3A-Mn1A-C6A & $64.36(16)$ \\
\hline C21A-Mn1A-C2A & $142.86(18)$ \\
\hline C20A-Mn1A-C2A & $90.36(18)$ \\
\hline C22A-Mn1A-C2A & $126.69(18)$ \\
\hline C4A-Mn1A-C2A & $63.31(16)$ \\
\hline C5A-Mn1A-C2A & $64.13(16)$ \\
\hline C3A-Mn1A-C2A & $38.26(15)$ \\
\hline C6A-Mn1A-C2A & $37.69(15)$ \\
\hline C7A-S1A-C1A & $96.2(2)$ \\
\hline C2A-C1A-C14A & $129.7(4)$ \\
\hline C2A-C1A-S1A & $109.0(3)$ \\
\hline C14A-C1A-S1A & $121.3(3)$ \\
\hline C1A-C2A-C3A & $139.7(4)$ \\
\hline C1A-C2A-C6A & $112.7(4)$ \\
\hline C3A-C2A-C6A & $107.6(4)$ \\
\hline C1A-C2A-Mn1A & $126.7(3)$ \\
\hline C3A-C2A-Mn1A & $66.6(2)$ \\
\hline C6A-C2A-Mn1A & $70.9(2)$ \\
\hline C4A-C3A-C2A & $106.9(4)$ \\
\hline C4A-C3A-Mn1A & $70.4(2)$ \\
\hline C2A-C3A-Mn1A & $75.1(2)$ \\
\hline C5A-C4A-C3A & $109.9(4)$ \\
\hline C5A-C4A-Mn1A & $70.8(2)$ \\
\hline C3A-C4A-Mn1A & $70.6(2)$ \\
\hline C4A-C5A-C6A & $107.7(4)$ \\
\hline C4A-C5A-Mn1A & $70.8(2)$ \\
\hline C6A-C5A-Mn1A & $74.9(2)$ \\
\hline C7A-C6A-C5A & $138.8(4)$ \\
\hline C7A-C6A-C2A & $113.9(4)$ \\
\hline C5A-C6A-C2A & $107.4(4)$ \\
\hline C7A-C6A-Mn1A & $126.1(3)$ \\
\hline C5A-C6A-Mn1A & $66.8(2)$ \\
\hline C2A-C6A-Mn1A & $71.4(2)$ \\
\hline C6A-C7A-C8A & $131.2(4)$ \\
\hline C6A-C7A-S1A & $108.2(3)$ \\
\hline C8A-C7A-S1A & $120.5(3)$ \\
\hline C13A-C8A-C9A & $117.6(4)$ \\
\hline C13A-C8A-C7A & $121.8(4)$ \\
\hline C9A-C8A-C7A & $120.6(4)$ \\
\hline C10A-C9A-C8A & $120.7(4)$ \\
\hline C11A-C10A-C9A & $121.0(4)$ \\
\hline
\end{tabular}

\begin{tabular}{|l|l|}
\hline C10A-C11A-C12A & $118.9(4)$ \\
\hline C13A-C12A-C11A & $120.9(4)$ \\
\hline C12A-C13A-C8A & $120.9(4)$ \\
\hline C19A-C14A-C15A & $117.1(4)$ \\
\hline C19A-C14A-C1A & $121.2(4)$ \\
\hline C15A-C14A-C1A & $121.7(4)$ \\
\hline C16A-C15A-C14A & $121.4(4)$ \\
\hline C16A-C17A-C18A & $120.3(4)$ \\
\hline C17A-C18A-C19A & $120.1(4)$ \\
\hline C18A-C19A-C14A & $121.4(4)$ \\
\hline O1A-C20A-Mn1A & $178.5(4)$ \\
\hline O2A-C21A-Mn1A & $179.0(4)$ \\
\hline O3A-C22A-Mn1A & $176.9(4)$ \\
\hline C21B-Mn1B-C20B & $89.36(19)$ \\
\hline C21B-Mn1B-C22B & $92.67(19)$ \\
\hline C20B-Mn1B-C22B & $95.8(2)$ \\
\hline C21B-Mn1B-C4B & $90.54(18)$ \\
\hline C20B-Mn1B-C4B & $129.03(18)$ \\
\hline C22B-Mn1B-C4B & $135.07(19)$ \\
\hline C21B-Mn1B-C3B & $106.06(18)$ \\
\hline C20B-Mn1B-C3B & $158.78(18)$ \\
\hline C22B-Mn1B-C3B & $97.87(19)$ \\
\hline C4B-Mn1B-C3B & $38.91(16)$ \\
\hline C21B-Mn1B-C5B & $111.47(17)$ \\
\hline C20B-Mn1B-C5B & $95.63(19)$ \\
\hline C22B-Mn1B-C5B & $153.33(17)$ \\
\hline C4B-Mn1B-C5B & $38.57(16)$ \\
\hline C3B-Mn1B-C5B & $65.44(17)$ \\
\hline C21B-Mn1B-C2B & $143.86(17)$ \\
\hline C20B-Mn1B-C2B & $126.24(18)$ \\
\hline C22B-Mn1B-C2B & $89.87(17)$ \\
\hline C4B-Mn1B-C2B & $63.40(16)$ \\
\hline C3B-Mn1B-C2B & $38.04(15)$ \\
\hline C5B-Mn1B-C2B & $64.03(16)$ \\
\hline C21B-Mn1B-C6B & $149.73(17)$ \\
\hline C20B-Mn1B-C6B & $95.30(18)$ \\
\hline C22B-Mn1B-C6B & $116.47(17)$ \\
\hline C4B-Mn1B-C6B & $63.48(16)$ \\
\hline C3B-Mn1B-C6B & $64.04(16)$ \\
\hline C5B-Mn1B-C6B & $38.35(15)$ \\
\hline C2B-Mn1B-C6B & $37.57(15)$ \\
\hline C1B-S1B-C7B & $96.5(2)$ \\
\hline C2B-C1B-C14B & $129.0(4)$ \\
\hline C2B-C1B-S1B & $108.5(3)$ \\
\hline C14B-C1B-S1B & $122.5(3)$ \\
\hline
\end{tabular}




\begin{tabular}{|l|l|}
\hline C1B-C2B-C3B & $139.4(4)$ \\
\hline C1B-C2B-C6B & $112.8(4)$ \\
\hline C3B-C2B-C6B & $107.7(4)$ \\
\hline C1B-C2B-Mn1B & $127.1(3)$ \\
\hline C3B-C2B-Mn1B & $66.6(2)$ \\
\hline C6B-C2B-Mn1B & $71.3(2)$ \\
\hline C4B-C3B-C2B & $107.6(4)$ \\
\hline C4B-C3B-Mn1B & $70.0(2)$ \\
\hline C2B-C3B-Mn1B & $75.4(2)$ \\
\hline C5B-C4B-C3B & $109.8(4)$ \\
\hline C5B-C4B-Mn1B & $71.4(2)$ \\
\hline C3B-C4B-Mn1B & $71.1(2)$ \\
\hline C4B-C5B-C6B & $107.7(4)$ \\
\hline C4B-C5B-Mn1B & $70.0(2)$ \\
\hline C6B-C5B-Mn1B & $75.1(2)$ \\
\hline C7B-C6B-C5B & $139.6(4)$ \\
\hline C7B-C6B-C2B & $113.4(4)$ \\
\hline C5B-C6B-C2B & $107.0(4)$ \\
\hline C7B-C6B-Mn1B & $128.1(3)$ \\
\hline C5B-C6B-Mn1B & $66.5(2)$ \\
\hline C2B-C6B-Mn1B & $71.1(2)$ \\
\hline C6B-C7B-C8B & $130.1(4)$ \\
\hline C6B-C7B-S1B & $108.8(3)$ \\
\hline C8B-C7B-S1B & $121.0(3)$ \\
\hline C9B-C8B-C13B & $118.1(4)$ \\
\hline C9B-C8B-C7B & $120.2(4)$ \\
\hline C13B-C8B-C7B & $121.6(4)$ \\
\hline C10B-C9B-C8B & $120.7(4)$ \\
\hline C11B-C10B-C9B & $120.2(4)$ \\
\hline C12B-C11B-C10B & $119.6(4)$ \\
\hline C11B-C12B-C13B & $121.2(4)$ \\
\hline C12B-C13B-C8B & $120.2(4)$ \\
\hline C15B-C14B-C19B & $116.9(4)$ \\
\hline C15B-C14B-C1B & $121.7(4)$ \\
\hline C19B-C14B-C1B & $121.4(4)$ \\
\hline C16B-C15B-C14B & $121.6(4)$ \\
\hline C15B-C16B-C17B & $120.2(4)$ \\
\hline C18B-C17B-C16B & $119.5(4)$ \\
\hline C17B-C18B-C19B & $120.4(4)$ \\
\hline C18B-C19B-C14B & $121.5(4)$ \\
\hline O1B-C20B-Mn1B & $177.1(4)$ \\
\hline O2B-C21B-Mn1B & $178.2(4)$ \\
\hline O3B-C22B-Mn1B & $177.7(4)$ \\
\hline
\end{tabular}


S36 
Table S10. Anisotropic displacement parameters $\left(\mathrm{A}^{2} \times 10^{3}\right)$ for $\left[\mathrm{Mn}\left\{\eta^{5}-\mathrm{SC}_{7} \mathrm{H}_{3}-1,3-\right.\right.$ $\left.\left.\mathrm{Ph}_{2}\right\}(\mathrm{CO})_{3}\right](3 \mathrm{c})$

\begin{tabular}{|c|c|c|c|c|c|c|}
\hline Atom & U11 & U22 & U33 & U23 & U13 & U12 \\
\hline Mn1A & 23(1) & $20(1)$ & 19(1) & $-10(1)$ & $-1(1)$ & $-3(1)$ \\
\hline S1A & $20(1)$ & $24(1)$ & 18(1) & $-9(1)$ & 1(1) & $-3(1)$ \\
\hline O1A & $45(2)$ & $34(2)$ & $28(2)$ & $-20(2)$ & $7(2)$ & $-12(2)$ \\
\hline $\mathrm{O} 2 \mathrm{~A}$ & $30(2)$ & $27(2)$ & $27(2)$ & $-12(2)$ & $-4(2)$ & 1(2) \\
\hline O3A & $32(2)$ & $25(2)$ & $31(2)$ & $-13(2)$ & $6(2)$ & $-10(2)$ \\
\hline C1A & $26(3)$ & $20(2)$ & $16(3)$ & $-10(2)$ & $4(2)$ & $-9(2)$ \\
\hline $\mathrm{C} 2 \mathrm{~A}$ & 21(3) & $13(2)$ & $23(3)$ & $-9(2)$ & $5(2)$ & $-7(2)$ \\
\hline C3A & $16(3)$ & $21(3)$ & $24(3)$ & $-13(2)$ & $4(2)$ & $-5(2)$ \\
\hline C4A & $28(3)$ & 19(3) & $23(3)$ & $-10(2)$ & $-6(2)$ & $-6(2)$ \\
\hline C5A & $25(3)$ & $24(3)$ & 19(3) & $-12(2)$ & 1(2) & $-10(2)$ \\
\hline C6A & 19(3) & 11(2) & $20(3)$ & $-6(2)$ & $-3(2)$ & $0(2)$ \\
\hline C7A & $27(3)$ & $16(2)$ & $15(2)$ & $-8(2)$ & 1(2) & $-4(2)$ \\
\hline $\mathrm{C} 8 \mathrm{~A}$ & 18(3) & 19(2) & 18(3) & $-11(2)$ & $2(2)$ & $-2(2)$ \\
\hline C9A & $23(3)$ & $28(3)$ & $26(3)$ & $-15(2)$ & 2(2) & $-9(2)$ \\
\hline C10A & $35(3)$ & $32(3)$ & $22(3)$ & $-16(2)$ & $7(2)$ & $-12(3)$ \\
\hline C11A & $21(3)$ & $28(3)$ & $32(3)$ & $-19(2)$ & $7(2)$ & $-8(2)$ \\
\hline C12A & $25(3)$ & $23(3)$ & $35(3)$ & $-14(2)$ & $-5(2)$ & $-2(2)$ \\
\hline C13A & 21(3) & $21(3)$ & $24(3)$ & $-11(2)$ & $2(2)$ & $-5(2)$ \\
\hline C14A & $29(3)$ & $10(2)$ & 19(3) & $-7(2)$ & $-2(2)$ & $-1(2)$ \\
\hline C15A & $20(3)$ & $24(3)$ & $29(3)$ & $-14(2)$ & $7(2)$ & $-11(2)$ \\
\hline C16A & $36(3)$ & $20(3)$ & $23(3)$ & $-11(2)$ & $3(2)$ & $-7(2)$ \\
\hline C17A & $30(3)$ & $23(3)$ & $21(3)$ & $-12(2)$ & $10(2)$ & $-10(2)$ \\
\hline C18A & $21(3)$ & $28(3)$ & $34(3)$ & $-16(2)$ & $7(2)$ & $-6(2)$ \\
\hline C19A & 21(3) & $28(3)$ & $23(3)$ & $-12(2)$ & 1(2) & $-2(2)$ \\
\hline C20A & 21(3) & 19(3) & $28(3)$ & $-3(2)$ & $-1(2)$ & $-9(2)$ \\
\hline $\mathrm{C} 21 \mathrm{~A}$ & $31(3)$ & $28(3)$ & $14(3)$ & $-11(2)$ & $3(2)$ & $-14(3)$ \\
\hline $\mathrm{C} 22 \mathrm{~A}$ & $24(3)$ & $20(3)$ & $17(3)$ & $-14(2)$ & $-4(2)$ & $8(2)$ \\
\hline Mn1B & $22(1)$ & 19(1) & 19(1) & $-10(1)$ & $2(1)$ & $-5(1)$ \\
\hline S1B & $20(1)$ & $21(1)$ & 15(1) & $-9(1)$ & $2(1)$ & $-6(1)$ \\
\hline O1B & $25(2)$ & $27(2)$ & $26(2)$ & $-12(2)$ & $-2(2)$ & $-1(2)$ \\
\hline O2B & $27(2)$ & $25(2)$ & 22(2) & $-8(2)$ & $3(2)$ & $-11(2)$ \\
\hline O3B & $46(2)$ & $32(2)$ & $21(2)$ & $-13(2)$ & $2(2)$ & $-15(2)$ \\
\hline C1B & $20(3)$ & $14(2)$ & $20(3)$ & $-9(2)$ & 1(2) & $-2(2)$ \\
\hline C2B & $19(3)$ & $14(2)$ & $25(3)$ & $-9(2)$ & $-1(2)$ & $-3(2)$ \\
\hline C3B & 19(3) & $24(3)$ & $25(3)$ & $-12(2)$ & $0(2)$ & $-2(2)$ \\
\hline C4B & $23(3)$ & $22(3)$ & $26(3)$ & $-17(2)$ & $7(2)$ & $-6(2)$ \\
\hline C5B & $28(3)$ & $18(2)$ & $15(2)$ & $-6(2)$ & $1(2)$ & $-6(2)$ \\
\hline C6B & $28(3)$ & $16(2)$ & $20(3)$ & $-12(2)$ & $4(2)$ & $-6(2)$ \\
\hline C7B & $22(3)$ & $19(2)$ & $14(2)$ & $-9(2)$ & 1(2) & $-5(2)$ \\
\hline C8B & $19(3)$ & $18(2)$ & $18(3)$ & $-13(2)$ & $5(2)$ & $-3(2)$ \\
\hline
\end{tabular}




\begin{tabular}{|l|l|l|l|l|l|l|}
\hline $\mathrm{C} 9 \mathrm{~B}$ & $24(3)$ & $23(3)$ & $19(3)$ & $-13(2)$ & $9(2)$ & $-10(2)$ \\
\hline $\mathrm{C} 10 \mathrm{~B}$ & $28(3)$ & $28(3)$ & $17(3)$ & $-11(2)$ & $-5(2)$ & $-5(2)$ \\
\hline $\mathrm{C} 11 \mathrm{~B}$ & $21(3)$ & $29(3)$ & $26(3)$ & $-15(2)$ & $-8(2)$ & $0(2)$ \\
\hline $\mathrm{C} 12 \mathrm{~B}$ & $28(3)$ & $30(3)$ & $26(3)$ & $-15(2)$ & $9(2)$ & $-12(2)$ \\
\hline $\mathrm{C} 13 \mathrm{~B}$ & $27(3)$ & $19(3)$ & $15(2)$ & $-8(2)$ & $1(2)$ & $-5(2)$ \\
\hline $\mathrm{C} 14 \mathrm{~B}$ & $23(3)$ & $16(2)$ & $21(3)$ & $-11(2)$ & $5(2)$ & $-3(2)$ \\
\hline $\mathrm{C} 15 \mathrm{~B}$ & $17(3)$ & $23(3)$ & $28(3)$ & $-14(2)$ & $-1(2)$ & $-4(2)$ \\
\hline $\mathrm{C} 16 \mathrm{~B}$ & $23(3)$ & $16(2)$ & $16(3)$ & $-7(2)$ & $-2(2)$ & $1(2)$ \\
\hline $\mathrm{C} 17 \mathrm{~B}$ & $25(3)$ & $20(3)$ & $20(3)$ & $-9(2)$ & $-3(2)$ & $0(2)$ \\
\hline $\mathrm{C} 18 \mathrm{~B}$ & $19(3)$ & $30(3)$ & $30(3)$ & $-15(2)$ & $-2(2)$ & $-7(2)$ \\
\hline $\mathrm{C} 19 \mathrm{~B}$ & $22(3)$ & $25(3)$ & $21(3)$ & $-6(2)$ & $1(2)$ & $-7(2)$ \\
\hline $\mathrm{C} 20 \mathrm{~B}$ & $20(3)$ & $30(3)$ & $18(3)$ & $-10(2)$ & $4(2)$ & $-13(2)$ \\
\hline $\mathrm{C} 21 \mathrm{~B}$ & $23(3)$ & $18(3)$ & $18(3)$ & $-13(2)$ & $-9(2)$ & $7(2)$ \\
\hline $\mathrm{C} 22 \mathrm{~B}$ & $25(3)$ & $17(3)$ & $22(3)$ & $-3(2)$ & $0(2)$ & $-6(2)$ \\
\hline
\end{tabular}

The anisotropic displacement factor exponent takes the form: $-2 \pi^{2}\left[h^{2} a^{* 2} U_{11}+\ldots+\right.$ $\left.2 \mathrm{hka}^{*} \mathrm{~b}^{*} \mathrm{U}_{12}\right]$. 
Table S11. Hydrogen coordinates $\left(\times 10^{4}\right)$ and isotropic displacement parameters $\left(A^{2} \times\right.$ $10^{3}$ ) for $\left[\mathrm{Mn}\left\{\eta^{5}-\mathrm{SC}_{7} \mathrm{H}_{3}-1,3-\mathrm{Ph}_{2}\right\}(\mathrm{CO})_{3}\right](\mathbf{3 c})$

\begin{tabular}{|l|l|l|l|l|}
\hline Atom & $x$ & $y$ & $z$ & $U(e q)$ \\
\hline H3A & 6151 & 5106 & 2914 & 23 \\
\hline H4A & 6632 & 5702 & 908 & 27 \\
\hline H5A & 8866 & 5743 & 632 & 25 \\
\hline H9A & 10803 & 5801 & 756 & 29 \\
\hline H10A & 12682 & 6053 & -54 & 33 \\
\hline H11A & 14487 & 5201 & 946 & 29 \\
\hline H12A & 14390 & 4128 & 2811 & 33 \\
\hline H13A & 12536 & 3834 & 3633 & 25 \\
\hline H15A & 9374 & 3310 & 6310 & 27 \\
\hline H16A & 8336 & 2637 & 7943 & 31 \\
\hline H17A & 6237 & 2758 & 7933 & 28 \\
\hline H18A & 5174 & 3506 & 6295 & 32 \\
\hline H19A & 6200 & 4175 & 4659 & 29 \\
\hline H3B & 5793 & 685 & 6770 & 27 \\
\hline H4B & 6039 & 5 & 8798 & 26 \\
\hline H5B & 8280 & -501 & 9256 & 24 \\
\hline H9B & 10371 & -1076 & 9229 & 24 \\
\hline H10B & 12165 & -1550 & 10213 & 28 \\
\hline H11B & 13853 & -799 & 9388 & 29 \\
\hline H12B & 13740 & 421 & 7583 & 32 \\
\hline H13B & 11978 & 893 & 6571 & 24 \\
\hline H15B & 9105 & 2093 & 3639 & 25 \\
\hline H16B & 8172 & 2835 & 1948 & 22 \\
\hline H17B & 6158 & 2706 & 1794 & 26 \\
\hline H18B & 5118 & 1762 & 3340 & 30 \\
\hline H19B & 6048 & 1005 & 5033 & 28 \\
\hline & & & & \\
\hline
\end{tabular}


Table S12. Torsion angles [deg] for $\left[\mathrm{Mn}\left\{\eta^{5}-\mathrm{SC}_{7} \mathrm{H}_{3}-1,3-\mathrm{Ph}_{2}\right\}(\mathrm{CO})_{3}\right](\mathbf{3 c})$

\begin{tabular}{|l|l|}
\hline C7A-S1A-C1A-C2A & $0.8(3)$ \\
\hline C7A-S1A-C1A-C14A & $178.7(3)$ \\
\hline C14A-C1A-C2A-C3A & $3.1(9)$ \\
\hline S1A-C1A-C2A-C3A & $-179.3(5)$ \\
\hline C14A-C1A-C2A-C6A & $-177.9(4)$ \\
\hline S1A-C1A-C2A-C6A & $-0.2(4)$ \\
\hline C14A-C1A-C2A-Mn1A & $99.6(5)$ \\
\hline S1A-C1A-C2A-Mn1A & $-82.7(4)$ \\
\hline C21A-Mn1A-C2A-C1A & $-123.4(4)$ \\
\hline C20A-Mn1A-C2A-C1A & $-31.3(4)$ \\
\hline C22A-Mn1A-C2A-C1A & $65.8(4)$ \\
\hline C4A-Mn1A-C2A-C1A & $-175.4(4)$ \\
\hline C5A-Mn1A-C2A-C1A & $141.5(4)$ \\
\hline C3A-Mn1A-C2A-C1A & $-135.6(5)$ \\
\hline C6A-Mn1A-C2A-C1A & $104.5(5)$ \\
\hline C21A-Mn1A-C2A-C3A & $12.2(4)$ \\
\hline C20A-Mn1A-C2A-C3A & $104.2(3)$ \\
\hline C22A-Mn1A-C2A-C3A & $-158.7(3)$ \\
\hline C4A-Mn1A-C2A-C3A & $-39.8(2)$ \\
\hline C5A-Mn1A-C2A-C3A & $-82.9(3)$ \\
\hline C6A-Mn1A-C2A-C3A & $-119.9(4)$ \\
\hline C21A-Mn1A-C2A-C6A & $132.0(3)$ \\
\hline C20A-Mn1A-C2A-C6A & $-135.9(3)$ \\
\hline C22A-Mn1A-C2A-C6A & $-38.8(3)$ \\
\hline C4A-Mn1A-C2A-C6A & $80.0(3)$ \\
\hline C5A-Mn1A-C2A-C6A & $37.0(2)$ \\
\hline C3A-Mn1A-C2A-C6A & $119.9(4)$ \\
\hline C1A-C2A-C3A-C4A & $-176.5(5)$ \\
\hline C6A-C2A-C3A-C4A & $4.4(5)$ \\
\hline Mn1A-C2A-C3A-C4A & $63.7(3)$ \\
\hline C1A-C2A-C3A-Mn1A & $119.8(6)$ \\
\hline C6A-C2A-C3A-Mn1A & $-59.3(3)$ \\
\hline C21A-Mn1A-C3A-C4A & $73.1(3)$ \\
\hline C20A-Mn1A-C3A-C4A & $166.6(3)$ \\
\hline C22A-Mn1A-C3A-C4A & $-61.0(6)$ \\
\hline C5A-Mn1A-C3A-C4A & $-36.0(3)$ \\
\hline C6A-Mn1A-C3A-C4A & $-78.5(3)$ \\
\hline C2A-Mn1A-C3A-C4A & $-114.5(4)$ \\
\hline C21A-Mn1A-C3A-C2A & $-172.4(3)$ \\
\hline C20A-Mn1A-C3A-C2A & $-79.0(3)$ \\
\hline C22A-Mn1A-C3A-C2A & $53.4(6)$ \\
\hline C4A-Mn1A-C3A-C2A & $114.5(4)$ \\
\hline
\end{tabular}

\begin{tabular}{|l|l|}
\hline C5A-Mn1A-C3A-C2A & $78.4(3)$ \\
\hline C6A-Mn1A-C3A-C2A & $36.0(2)$ \\
\hline C2A-C3A-C4A-C5A & $-6.8(5)$ \\
\hline Mn1A-C3A-C4A-C5A & $60.1(3)$ \\
\hline C2A-C3A-C4A-Mn1A & $-66.9(3)$ \\
\hline C21A-Mn1A-C4A-C5A & $127.2(3)$ \\
\hline C20A-Mn1A-C4A-C5A & $-139.8(3)$ \\
\hline C22A-Mn1A-C4A-C5A & $35.9(3)$ \\
\hline C3A-Mn1A-C4A-C5A & $-120.4(4)$ \\
\hline C6A-Mn1A-C4A-C5A & $-38.8(2)$ \\
\hline C2A-Mn1A-C4A-C5A & $-81.2(3)$ \\
\hline C21A-Mn1A-C4A-C3A & $-112.5(3)$ \\
\hline C20A-Mn1A-C4A-C3A & $-19.5(4)$ \\
\hline C22A-Mn1A-C4A-C3A & $156.3(3)$ \\
\hline C5A-Mn1A-C4A-C3A & $120.4(4)$ \\
\hline C6A-Mn1A-C4A-C3A & $81.5(3)$ \\
\hline C2A-Mn1A-C4A-C3A & $39.1(2)$ \\
\hline C3A-C4A-C5A-C6A & $6.4(5)$ \\
\hline Mn1A-C4A-C5A-C6A & $66.4(3)$ \\
\hline C3A-C4A-C5A-Mn1A & $-60.0(3)$ \\
\hline C21A-Mn1A-C5A-C4A & $-60.2(3)$ \\
\hline C20A-Mn1A-C5A-C4A & $95.2(4)$ \\
\hline C22A-Mn1A-C5A-C4A & $-152.3(3)$ \\
\hline C3A-Mn1A-C5A-C4A & $36.5(3)$ \\
\hline C6A-Mn1A-C5A-C4A & $115.3(4)$ \\
\hline C2A-Mn1A-C5A-C4A & $78.9(3)$ \\
\hline C21A-Mn1A-C5A-C6A & $-175.5(2)$ \\
\hline C20A-Mn1A-C5A-C6A & $-20.2(5)$ \\
\hline C22A-Mn1A-C5A-C6A & $92.4(3)$ \\
\hline C4A-Mn1A-C5A-C6A & $-115.3(4)$ \\
\hline C3A-Mn1A-C5A-C6A & $-78.8(3)$ \\
\hline C2A-Mn1A-C5A-C6A & $-36.4(2)$ \\
\hline C4A-C5A-C6A-C7A & $177.5(5)$ \\
\hline Mn1A-C5A-C6A-C7A & $-118.8(5)$ \\
\hline C4A-C5A-C6A-C2A & $-3.5(5)$ \\
\hline Mn1A-C5A-C6A-C2A & $60.1(3)$ \\
\hline C4A-C5A-C6A-Mn1A & $-63.6(3)$ \\
\hline C1A-C2A-C6A-C7A & $-0.7(5)$ \\
\hline C3A-C2A-C6A-C7A & $178.6(3)$ \\
\hline Mn1A-C2A-C6A-C7A & $122.0(3)$ \\
\hline C1A-C2A-C6A-C5A & $180.0(3)$ \\
\hline C3A-C2A-C6A-C5A & $-0.6(4)$ \\
\hline
\end{tabular}




\begin{tabular}{|l|l|}
\hline Mn1A-C2A-C6A-C5A & $-57.2(3)$ \\
\hline C1A-C2A-C6A-Mn1A & $-122.8(3)$ \\
\hline C3A-C2A-C6A-Mn1A & $56.6(3)$ \\
\hline C21A-Mn1A-C6A-C7A & $143.1(4)$ \\
\hline C20A-Mn1A-C6A-C7A & $-55.4(4)$ \\
\hline C22A-Mn1A-C6A-C7A & $43.3(4)$ \\
\hline C4A-Mn1A-C6A-C7A & $173.4(4)$ \\
\hline C5A-Mn1A-C6A-C7A & $134.4(5)$ \\
\hline C3A-Mn1A-C6A-C7A & $-143.0(4)$ \\
\hline C2A-Mn1A-C6A-C7A & $-106.4(5)$ \\
\hline C21A-Mn1A-C6A-C5A & $8.7(5)$ \\
\hline C20A-Mn1A-C6A-C5A & $170.1(3)$ \\
\hline C22A-Mn1A-C6A-C5A & $-91.1(3)$ \\
\hline C4A-Mn1A-C6A-C5A & $39.0(2)$ \\
\hline C3A-Mn1A-C6A-C5A & $82.6(3)$ \\
\hline C2A-Mn1A-C6A-C5A & $119.2(3)$ \\
\hline C21A-Mn1A-C6A-C2A & $-110.5(4)$ \\
\hline C20A-Mn1A-C6A-C2A & $51.0(3)$ \\
\hline C22A-Mn1A-C6A-C2A & $149.7(3)$ \\
\hline C4A-Mn1A-C6A-C2A & $-80.2(3)$ \\
\hline C5A-Mn1A-C6A-C2A & $-119.2(3)$ \\
\hline C3A-Mn1A-C6A-C2A & $-36.5(2)$ \\
\hline C5A-C6A-C7A-C8A & $1.8(9)$ \\
\hline C2A-C6A-C7A-C8A & $-177.1(4)$ \\
\hline Mn1A-C6A-C7A-C8A & $-93.4(5)$ \\
\hline C5A-C6A-C7A-S1A & $-179.8(4)$ \\
\hline C2A-C6A-C7A-S1A & $1.3(4)$ \\
\hline Mn1A-C6A-C7A-S1A & $85.0(4)$ \\
\hline C1A-S1A-C7A-C6A & $-1.2(3)$ \\
\hline C1A-S1A-C7A-C8A & $177.4(3)$ \\
\hline C6A-C7A-C8A-C13A & $-172.2(4)$ \\
\hline S1A-C7A-C8A-C13A & $9.6(6)$ \\
\hline C6A-C7A-C8A-C9A & $10.6(7)$ \\
\hline S1A-C7A-C8A-C9A & $-167.6(3)$ \\
\hline C13A-C8A-C9A-C10A & $0.8(6)$ \\
\hline C7A-C8A-C9A-C10A & $178.1(4)$ \\
\hline C8A-C9A-C10A-C11A & $-0.2(7)$ \\
\hline C9A-C10A-C11A-C12A & $-1.1(7)$ \\
\hline C10A-C11A-C12A-C13A & $1.8(7)$ \\
\hline C11A-C12A-C13A-C8A & $-1.1(7)$ \\
\hline C9A-C8A-C13A-C12A & $-0.26)$ \\
\hline C7A-C8A-C13A-C12A & $-177.5(4)$ \\
\hline C2A-C1A-C14A-C19A & $2.7(7)$ \\
\hline S1A-C1A-C14A-C19A & $-174.7(3)$ \\
\hline C2A-C1A-C14A-C15A & $-178.5(4)$ \\
\hline
\end{tabular}

\begin{tabular}{|l|l|}
\hline S1A-C1A-C14A-C15A & $4.1(6)$ \\
\hline C19A-C14A-C15A-C16A & $0.4(6)$ \\
\hline C1A-C14A-C15A-C16A & $-178.4(4)$ \\
\hline C14A-C15A-C16A-C17A & $-0.7(7)$ \\
\hline C15A-C16A-C17A-C18A & $0.8(7)$ \\
\hline C16A-C17A-C18A-C19A & $-0.7(7)$ \\
\hline C17A-C18A-C19A-C14A & $0.4(7)$ \\
\hline C15A-C14A-C19A-C18A & $-0.3(6)$ \\
\hline C1A-C14A-C19A-C18A & $178.6(4)$ \\
\hline C21A-Mn1A-C20A-O1A & $108(18)$ \\
\hline C22A-Mn1A-C20A-O1A & $-162(18)$ \\
\hline C4A-Mn1A-C20A-O1A & $15(18)$ \\
\hline C5A-Mn1A-C20A-O1A & $-50(18)$ \\
\hline C3A-Mn1A-C20A-O1A & $2(18)$ \\
\hline C6A-Mn1A-C20A-O1A & $-64(18)$ \\
\hline C2A-Mn1A-C20A-O1A & $-35(18)$ \\
\hline C20A-Mn1A-C21A-O2A & $-119(25)$ \\
\hline C22A-Mn1A-C21A-O2A & $146(25)$ \\
\hline C4A-Mn1A-C21A-O2A & $18(25)$ \\
\hline C5A-Mn1A-C21A-O2A & $51(25)$ \\
\hline C3A-Mn1A-C21A-O2A & $-19(25)$ \\
\hline C6A-Mn1A-C21A-O2A & $45(26)$ \\
\hline C2A-Mn1A-C21A-O2A & $-27(26)$ \\
\hline C21A-Mn1A-C22A-O3A & $-66(7)$ \\
\hline C20A-Mn1A-C22A-O3A & $-157(7)$ \\
\hline C4A-Mn1A-C22A-O3A & $25(7)$ \\
\hline C5A-Mn1A-C22A-O3A & $47(7)$ \\
\hline C3A-Mn1A-C22A-O3A & $70(7)$ \\
\hline C6A-Mn1A-C22A-O3A & $85(7)$ \\
\hline C2A-Mn1A-C22A-O3A & $108(7)$ \\
\hline C7B-S1B-C1B-C2B & $0.9(3)$ \\
\hline C7B-S1B-C1B-C14B & $-179.9(4)$ \\
\hline C14B-C1B-C2B-C3B & $-0.3(9)$ \\
\hline S1B-C1B-C2B-C3B & $178.8(5)$ \\
\hline C14B-C1B-C2B-C6B & $179.7(4)$ \\
\hline S1B-C1B-C2B-C6B & $-1.2(4)$ \\
\hline C14B-C1B-C2B-Mn1B & $96.4(5)$ \\
\hline S1B-C1B-C2B-Mn1B & $-84.5(4)$ \\
\hline C21B-Mn1B-C2B-C1B & $-126.6(4)$ \\
\hline C20B-Mn1B-C2B-C1B & $64.9(4)$ \\
\hline C22B-Mn1B-C2B-C1B & $-32.3(4)$ \\
\hline C4B-Mn1B-C2B-C1B & $-174.7(4)$ \\
\hline C3B-Mn1B-C2B-C1B & $-135.3(5)$ \\
\hline C5B-Mn1B-C2B-C1B & $142.1(4)$ \\
\hline C6B-Mn1B-C2B-C1B & $105.0(5)$ \\
\hline
\end{tabular}




\begin{tabular}{|l|l|}
\hline C21B-Mn1B-C2B-C3B & $8.7(4)$ \\
\hline C20B-Mn1B-C2B-C3B & $-159.9(3)$ \\
\hline C22B-Mn1B-C2B-C3B & $103.0(3)$ \\
\hline C4B-Mn1B-C2B-C3B & $-39.4(2)$ \\
\hline C5B-Mn1B-C2B-C3B & $-82.7(3)$ \\
\hline C6B-Mn1B-C2B-C3B & $-119.8(4)$ \\
\hline C21B-Mn1B-C2B-C6B & $128.4(3)$ \\
\hline C20B-Mn1B-C2B-C6B & $-40.1(3)$ \\
\hline C22B-Mn1B-C2B-C6B & $-137.2(3)$ \\
\hline C4B-Mn1B-C2B-C6B & $80.3(3)$ \\
\hline C3B-Mn1B-C2B-C6B & $119.8(4)$ \\
\hline C5B-Mn1B-C2B-C6B & $37.1(2)$ \\
\hline C1B-C2B-C3B-C4B & $-176.6(5)$ \\
\hline C6B-C2B-C3B-C4B & $3.4(5)$ \\
\hline Mn1B-C2B-C3B-C4B & $63.1(3)$ \\
\hline C1B-C2B-C3B-Mn1B & $120.3(6)$ \\
\hline C6B-C2B-C3B-Mn1B & $-59.7(3)$ \\
\hline C21B-Mn1B-C3B-C4B & $70.1(3)$ \\
\hline C20B-Mn1B-C3B-C4B & $-65.1(6)$ \\
\hline C22B-Mn1B-C3B-C4B & $165.1(3)$ \\
\hline C5B-Mn1B-C3B-C4B & $-36.6(2)$ \\
\hline C2B-Mn1B-C3B-C4B & $-115.2(4)$ \\
\hline C6B-Mn1B-C3B-C4B & $-79.2(3)$ \\
\hline C21B-Mn1B-C3B-C2B & $-174.7(3)$ \\
\hline C20B-Mn1B-C3B-C2B & $50.1(6)$ \\
\hline C22B-Mn1B-C3B-C2B & $-79.6(3)$ \\
\hline C4B-Mn1B-C3B-C2B & $115.2(4)$ \\
\hline C5B-Mn1B-C3B-C2B & $78.6(3)$ \\
\hline C6B-Mn1B-C3B-C2B & $36.1(2)$ \\
\hline C2B-C3B-C4B-C5B & $-5.4(5)$ \\
\hline Mn1B-C3B-C4B-C5B & $61.3(3)$ \\
\hline C2B-C3B-C4B-Mn1B & $-66.6(3)$ \\
\hline C21B-Mn1B-C4B-C5B & $125.1(3)$ \\
\hline C20B-Mn1B-C4B-C5B & $35.5(3)$ \\
\hline C22B-Mn1B-C4B-C5B & $-140.6(3)$ \\
\hline C3B-Mn1B-C4B-C5B & $-119.5(4)$ \\
\hline C2B-Mn1B-C4B-C5B & $-80.9(3)$ \\
\hline C6B-Mn1B-C4B-C5B & $-38.7(2)$ \\
\hline C21B-Mn1B-C4B-C3B & $-115.4(3)$ \\
\hline C20B-Mn1B-C4B-C3B & $155.0(3)$ \\
\hline C22B-Mn1B-C4B-C3B & $-21.1(4)$ \\
\hline C5B-Mn1B-C4B-C3B & $119.5(4)$ \\
\hline C2B-Mn1B-C4B-C3B & $38.6(2)$ \\
\hline C6B-Mn1B-C4B-C3B & $80.8(3)$ \\
\hline C3B-C4B-C5B-C6B & $5.2(5)$ \\
\hline
\end{tabular}

\begin{tabular}{|l|l|}
\hline Mn1B-C4B-C5B-C6B & \multicolumn{1}{|c|}{$66.3(3)$} \\
\hline C3B-C4B-C5B-Mn1B & $-61.0(3)$ \\
\hline C21B-Mn1B-C5B-C4B & $-61.5(3)$ \\
\hline C20B-Mn1B-C5B-C4B & $-153.0(3)$ \\
\hline C22B-Mn1B-C5B-C4B & $91.9(5)$ \\
\hline C3B-Mn1B-C5B-C4B & $37.0(2)$ \\
\hline C2B-Mn1B-C5B-C4B & $79.2(3)$ \\
\hline C6B-Mn1B-C5B-C4B & $115.5(4)$ \\
\hline C21B-Mn1B-C5B-C6B & $-177.0(3)$ \\
\hline C20B-Mn1B-C5B-C6B & $91.4(3)$ \\
\hline C22B-Mn1B-C5B-C6B & $-23.6(5)$ \\
\hline C4B-Mn1B-C5B-C6B & $-115.5(4)$ \\
\hline C3B-Mn1B-C5B-C6B & $-78.6(3)$ \\
\hline C2B-Mn1B-C5B-C6B & $-36.4(2)$ \\
\hline C4B-C5B-C6B-C7B & $175.3(5)$ \\
\hline Mn1B-C5B-C6B-C7B & $-121.8(6)$ \\
\hline C4B-C5B-C6B-C2B & $-3.0(5)$ \\
\hline Mn1B-C5B-C6B-C2B & $59.8(3)$ \\
\hline C4B-C5B-C6B-Mn1B & $-62.9(3)$ \\
\hline C1B-C2B-C6B-C7B & $1.0(5)$ \\
\hline C3B-C2B-C6B-C7B & $-179.0(4)$ \\
\hline Mn1B-C2B-C6B-C7B & $124.2(3)$ \\
\hline C1B-C2B-C6B-C5B & $179.8(3)$ \\
\hline C3B-C2B-C6B-C5B & $-0.2(5)$ \\
\hline Mn1B-C2B-C6B-C5B & $-57.0(3)$ \\
\hline C1B-C2B-C6B-Mn1B & $-123.3(3)$ \\
\hline C3B-C2B-C6B-Mn1B & $56.7(3)$ \\
\hline C21B-Mn1B-C6B-C7B & $141.1(4)$ \\
\hline C20B-Mn1B-C6B-C7B & $43.2(4)$ \\
\hline C22B-Mn1B-C6B-C7B & $-56.0(4)$ \\
\hline C4B-Mn1B-C6B-C7B & $174.6(5)$ \\
\hline C3B-Mn1B-C6B-C7B & $-141.8(4)$ \\
\hline C5B-Mn1B-C6B-C7B & $135.6(5)$ \\
\hline C2B-Mn1B-C6B-C7B & $-105.3(5)$ \\
\hline C21B-Mn1B-C6B-C5B & $5.5(5)$ \\
\hline C20B-Mn1B-C6B-C5B & $-92.4(3)$ \\
\hline C22B-Mn1B-C6B-C5B & $168.4(3)$ \\
\hline C4B-Mn1B-C6B-C5B & $39.0(3)$ \\
\hline C3B-Mn1B-C6B-C5B & $82.5(3)$ \\
\hline C2B-Mn1B-C6B-C5B & $119.1(4)$ \\
\hline C21B-Mn1B-C6B-C2B & $-113.6(4)$ \\
\hline C20B-Mn1B-C6B-C2B & $148.6(3)$ \\
\hline C22B-Mn1B-C6B-C2B & $49.4(3)$ \\
\hline C4B-Mn1B-C6B-C2B & $-80.1(3)$ \\
\hline C3B-Mn1B-C6B-C2B & $-36.5(2)$ \\
\hline
\end{tabular}




\begin{tabular}{|l|l|}
\hline C5B-Mn1B-C6B-C2B & $-119.1(4)$ \\
\hline C5B-C6B-C7B-C8B & $-1.0(9)$ \\
\hline C2B-C6B-C7B-C8B & $177.2(4)$ \\
\hline Mn1B-C6B-C7B-C8B & $-99.1(5)$ \\
\hline C5B-C6B-C7B-S1B & $-178.5(5)$ \\
\hline C2B-C6B-C7B-S1B & $-0.2(4)$ \\
\hline Mn1B-C6B-C7B-S1B & $83.5(4)$ \\
\hline C1B-S1B-C7B-C6B & $-0.4(3)$ \\
\hline C1B-S1B-C7B-C8B & $-178.2(3)$ \\
\hline C6B-C7B-C8B-C9B & $21.0(7)$ \\
\hline S1B-C7B-C8B-C9B & $-161.8(3)$ \\
\hline C6B-C7B-C8B-C13B & $-160.8(4)$ \\
\hline S1B-C7B-C8B-C13B & $16.4(6)$ \\
\hline C13B-C8B-C9B-C10B & $1.3(6)$ \\
\hline C7B-C8B-C9B-C10B & $179.5(4)$ \\
\hline C8B-C9B-C10B-C11B & $-0.3(7)$ \\
\hline C9B-C10B-C11B-C12B & $-0.2(7)$ \\
\hline C10B-C11B-C12B-C13B & $-0.2(7)$ \\
\hline C11B-C12B-C13B-C8B & $1.2(7)$ \\
\hline C9B-C8B-C13B-C12B & $-1.7(6)$ \\
\hline C7B-C8B-C13B-C12B & $-179.9(4)$ \\
\hline C2B-C1B-C14B-C15B & $166.8(4)$ \\
\hline S1B-C1B-C14B-C15B & $-12.2(6)$ \\
\hline C2B-C1B-C14B-C19B & $-14.7(7)$ \\
\hline S1B-C1B-C14B-C19B & $166.3(3)$ \\
\hline C19B-C14B-C15B-C16B & $1.3(6)$ \\
\hline C1B-C14B-C15B-C16B & $179.8(4)$ \\
\hline C14B-C15B-C16B-C17B & $0.2(6)$ \\
\hline C15B-C16B-C17B-C18B & $-1.4(6)$ \\
\hline C16B-C17B-C18B-C19B & $1.2(7)$ \\
\hline C17B-C18B-C19B-C14B & $0.4(7)$ \\
\hline C15B-C14B-C19B-C18B & $-1.6(6)$ \\
\hline C1B-C14B-C19B-C18B & $179.9(4)$ \\
\hline C21B-Mn1B-C20B-O1B & $-29(8)$ \\
\hline C22B-Mn1B-C20B-O1B & $-121(8)$ \\
\hline C4B-Mn1B-C20B-O1B & $62(8)$ \\
\hline C3B-Mn1B-C20B-O1B & $109(8)$ \\
\hline C5B-Mn1B-C20B-O1B & $83(8)$ \\
\hline C2B-Mn1B-C20B-01B & $145(8)$ \\
\hline C6B-Mn1B-C20B-O1B & $122(8)$ \\
\hline C20B-Mn1B-C21B-O2B & $73(13)$ \\
\hline C22B-Mn1B-C21B-O2B & $168(13)$ \\
\hline C4B-Mn1B-C21B-O2B & $-56(13)$ \\
\hline C3B-Mn1B-C21B-O2B & $-93(13)$ \\
\hline C5B-Mn1B-C21B-O2B & $-23(13)$ \\
\hline
\end{tabular}

\begin{tabular}{|l|l|}
\hline C2B-Mn1B-C21B-O2B & $-98(13)$ \\
\hline C6B-Mn1B-C21B-O2B & $-27(13)$ \\
\hline C21B-Mn1B-C22B-O3B & $136(10)$ \\
\hline C20B-Mn1B-C22B-O3B & $-134(10)$ \\
\hline C4B-Mn1B-C22B-O3B & $43(10)$ \\
\hline C3B-Mn1B-C22B-O3B & $30(10)$ \\
\hline C5B-Mn1B-C22B-O3B & $-19(10)$ \\
\hline C2B-Mn1B-C22B-O3B & $-8(10)$ \\
\hline C6B-Mn1B-C22B-O3B & $-35(10)$ \\
\hline
\end{tabular}

